\title{
A checklist of chromosome numbers and a review of karyotype variation in Odonata of the world
}

\author{
Valentina G. Kuznetsova', Natalia V. Golub' \\ I Department of Karyosystematics, Zoological Institute, Russian Academy of Sciences, Universitetskaya emb. 1, \\ St. Petersburg 199034, Russia \\ Corresponding author: Valentina G. Kuznetsova (valentina_kuznetsova@yahoo.com)
}

Academic editor: S. Grozeva | Received 29 July 2020 | Accepted 12 September 2020 | Published 22 October 2020

http://zoobank.org/4F8C7E6B-B7E7-483E-9794-0440845307CD

Citation: Kuznetsova VG, Golub NV (2020) A checklist of chromosome numbers and a review of karyotype variation in Odonata of the world. CompCytogen 14(4): 501-540. https://doi.org/10.3897/compcytogen.v14.i4.57062

\begin{abstract}
The ancient insect order Odonata is divided into three suborders: Anisoptera and Zygoptera with approximately 3000 species worldwide each, and Anisozygoptera with only four extant species in the relict family Epiophlebiidae. An updated list of Odonata species studied regarding chromosome number, sex chromosome mechanism and the occurrence of m-chromosomes (= microchromosomes) is given. Karyotypes of 607 species (198 genera, 23 families), covering approximately 10\% of described species, are reported: 423 species (125 genera, 8 families) of the Anisoptera, 184 species (72 genera, 14 families) of the Zygoptera, and one species of the Anisozygoptera. Among the Odonata, sex determination mechanisms in males can be of $\mathrm{X}(0), \mathrm{XY}$ and $\mathrm{X}_{1} \mathrm{X}_{2} \mathrm{Y}$ types, and diploid chromosome numbers can vary from 6 to 41, with a clear mode at $2 \mathrm{n}=25(60 \%)$ and two more local modes at $2 \mathrm{n}=27(21 \%)$ and $2 \mathrm{n}=23(13 \%)$. The karyotype $2 \mathrm{n}=25(24 \mathrm{~A}+\mathrm{X})$ is found in each of the three suborders and is the most typical (modal) in many families, including the best-covered Libellulidae, Corduliidae (Anisoptera), Lestidae, Calopterygidae, and Platycnemididae (Zygoptera). This chromosome set is considered ancestral for the Odonata in general. Chromosome rearrangements, among which fusions and fissions most likely
\end{abstract}

Copyright Valentina G. Kuznetsova, Natalia V. Golub. This is an open access article distributed under the terms of the Creative Commons Attribution License (CC BY 4.0), which permits unrestricted use, distribution, and reproduction in any medium, provided the original author and source are credited. 
predominated, led to independent origins of similar karyotypes within different phylogenetic lineages of the order. The karyotype $2 n=27(26 \mathrm{~A}+\mathrm{X})$ prevails in Aeshnidae and Coenagrionidae, whereas the karyotype $2 \mathrm{n}=23(22 \mathrm{~A}+\mathrm{X})$ is modal in Gomphidae and Chlorocyphidae, in both pairs of families one being from the Anisoptera while the other from the Zygoptera.

\section{Keywords}

Chromosome numbers, damseldragons, damselflies, dragonflies, m-chromosomes, sex chromosome mechanisms

\section{Introduction}

The order Odonata, which comprises slightly more than 6,000 described species worldwide, is one of the most ancient among winged insects (Pterygota), dating from the Permian (Grimaldi and Engel 2005). Extant Odonata include two main suborders with approximately 3,000 species each, the Zygoptera or damselflies with about 308 genera and the Anisoptera or true dragonflies with about 344 genera. Within these suborders, up to 21 and 11 families (and sometimes more), respectively, are currently recognized. The third suborder, the Anisozygoptera or damseldragons, includes only one genus Epiophlebia Calvert, 1903 with four extant species in the relict family Epiophlebiidae. A substantial body of evidence indicates that Anisoptera and Zygoptera are each monophyletic, and Zygoptera are sister to Epiophlebia plus Anisoptera (Rehn 2003; Kalkman et al. 2008; Dijkstra et al. 2013, 2014; Schorr and Paulson 2020).

The field of Odonata cytogenetics was heavily influenced by Bastiaan Kiauta, who has published dozens of papers and analyzed karyotypes of about 260 species and subspecies of this group (see References and Table 1). During the years that have passed since the publication of chromosome number checklist of Odonata (Kiauta 1972c), approximately 90 chromosome papers have been published. The number of examined species has since increased by more than 2.3 times, and now it seems appropriate to publish an updated list. In this review article, all data available today are presented in two tables and one figure. Table 1 includes all species studied so far cytogenetically and compiles data on their chromosome numbers, sex chromosome mechanisms and the occurrence of the so-called m-chromosomes (= microchromosomes). Table 2 summarizes data presented in Table 1 and shows the family-level variability of the above-mentioned traits (except m-chromosomes, since data on their presence or absence in specific species are often questionable) together with the most characteristic (modal) karyotypes for each of the families explored. On the Fig. 1, the modal karyotypes are mapped onto phylogenetic tree of Odonata families taken from Bybee et al. (2016) who in turn redrawn and synthesized it from Dijkstra et al. (2014) and Carle et al. (2015). In the final section of the review, the main characteristics of Odonata karyotypes are briefly discussed and prospects for future research are outlined. 
Table I. Cytogenetically analyzed species of Odonata and their main karyotype characteristics (chromosome numbers, sex chromosomes, m-chromosomes).

\begin{tabular}{|c|c|c|c|c|c|}
\hline \multicolumn{2}{|c|}{ Taxon } & $\begin{array}{c}\text { Karyotype } \\
\text { formula 2n } ठ\end{array}$ & $\begin{array}{c}\text { m-chromo } \\
\text { somes }\end{array}$ & Country & References \\
\hline \multicolumn{6}{|c|}{ ANISOZYGOPTERA } \\
\hline \multicolumn{6}{|c|}{ EPIOPHLEBIOIDEA } \\
\hline \multicolumn{6}{|c|}{ Epiophlebiidae } \\
\hline 1. & Epiophlebia superstes Selys, 1889 & $25(24 \mathrm{~A}+\mathrm{X})$ & - & Japan & Oguma 1951 \\
\hline \multicolumn{6}{|c|}{ ANISOPTERA } \\
\hline \multicolumn{6}{|c|}{ AESHNOIDEA } \\
\hline \multicolumn{6}{|c|}{ Aeshnidae } \\
\hline 2. & Aeshna caerulea (Ström, 1783) & $24(22 \mathrm{~A}+$ neo-XY $)$ & - & Finland & Oksala 1943 \\
\hline 3. & A. canadiensis Walker, 1908 & $27(26 \mathrm{~A}+\mathrm{X})$ & + & USA & Cruden 1968 \\
\hline 4. & A. clepsydra Say, 1839 & $27(26 \mathrm{~A}+\mathrm{X})$ & + & USA & Hung 1971 \\
\hline \multirow[t]{2}{*}{5.} & \multirow{2}{*}{ A. crenata Hagen, 1856} & $27(26 \mathrm{~A}+\mathrm{X})$ & + & Finland & Oksala 1939a, 1943, 1944, 1952 \\
\hline & & $-n-$ & - & Russia & Perepelov and Bugrov 2002 \\
\hline \multirow[t]{2}{*}{6.} & \multirow[t]{2}{*}{ A. cyanea (Müller, 1764) } & $27(26 \mathrm{~A}+\mathrm{X})$ & + & Finland & Oksala 1943 \\
\hline & & $-n-$ & + & Netherlands & Kiauta 1969a \\
\hline \multirow[t]{6}{*}{7.} & \multirow[t]{6}{*}{ A. grandis (Linnaeus, 1758) } & $27(26 \mathrm{~A}+\mathrm{X})$ & + & Former USSR & Fuchsówna and Sawczyńska 1928 \\
\hline & & $25(24 \mathrm{~A}+\mathrm{X})$ & + & Former USSR & Makalowskaja 1940 \\
\hline & & $26(24 \mathrm{~A}+$ neo-XY $)$ & + & Finland & Oksala 1939a, 1943, 1944, 1945 \\
\hline & & $-»-$ & + & Netherlands & Kiauta 1967a-d 1968a, b, 1969a \\
\hline & & $-»-$ & + & Russia & Perepelov and Bugrov 2002 \\
\hline & & $25(24 \mathrm{~A}+\mathrm{X})$ & - & Finland & Nokkala et al. 2002 \\
\hline \multirow[t]{2}{*}{8.} & \multirow[t]{2}{*}{ A. isoceles (Müller, 1767) } & $27(26 \mathrm{~A}+\mathrm{X})$ & - & USA & $\begin{array}{c}\text { Kiauta } 1978 \text { as Anaciaeschna isosceles } \\
\text { (Müller, 1767) }\end{array}$ \\
\hline & & $25(24 \mathrm{~A}+\mathrm{X})$ & + & Russia & Kuznetsova et al. 2020b \\
\hline \multirow[t]{4}{*}{9.} & \multirow[t]{4}{*}{ A. juncea (Linnaeus, 1758) } & $26(24 \mathrm{~A}+$ neo-XY $)$ & + & Finland & Oksala 1939a, 1943, 1944 \\
\hline & & $-»-$ & + & Former USSR & Makalowskaja 1940 \\
\hline & & $27(26 \mathrm{~A}+\mathrm{X})$ & + & Italy & Kiauta 1971a \\
\hline & & $26(24 \mathrm{~A}+$ neo-XY $)$ & + & Russia & Perepelov and Bugrov 2002 \\
\hline \multirow[t]{4}{*}{10.} & \multirow[t]{4}{*}{ A. mixta Latreille, 1805} & $27(26 \mathrm{~A}+\mathrm{X})$ & + & Netherlands & Kiauta $1969 \mathrm{a}$ \\
\hline & & $25(24 \mathrm{~A}+\mathrm{X})$ & + & India & Sandhu and Malhotra 1994a \\
\hline & & $-»-$ & + & India & Sharma and Durani 1995 \\
\hline & & $27(26 \mathrm{~A}+\mathrm{X})$ & + & Russia & Perepelov and Bugrov 2001b \\
\hline \multirow[t]{2}{*}{11.} & \multirow[t]{2}{*}{ A. nigroflava Martin, 1909} & $27(26 \mathrm{~A}+\mathrm{X})$ & + & Japan & Katatani 1987 \\
\hline & & $-»-$ & - & Russia & Perepelov and Bugrov 2002 \\
\hline 12. & A. palmata Hagen, 1856 & $27(26 \mathrm{~A}+\mathrm{X})$ & + & USA & Cruden 1968 \\
\hline 13. & A. serrata Hagen, 1856 & $26(24 \mathrm{~A}+$ neo-XY $)$ & + & Finland & $\begin{array}{c}\text { Oksala } 1943 \text { as } \\
\text { A. osiliensis Mierzejewski, } 1913 \text { and } \\
\text { A. s. fennica Valle, } 1938\end{array}$ \\
\hline 14. & A. subarctica Walker, 1908 & $27(26 \mathrm{~A}+\mathrm{X})$ & + & USA & $\begin{array}{c}\text { Oksala 1939a, 1943, } 1952 \text { as } \\
\text { A. s. elisabethae Djakonov, } 1922\end{array}$ \\
\hline & & $-»-$ & + & Switzerland & $\begin{array}{c}\text { Kiauta and Kiauta } 1980 \mathrm{a} \text { as } \\
\text { A. s. elisabethae }\end{array}$ \\
\hline 15. & A. umbrosa Walker, 1908 & $27(26 \mathrm{~A}+\mathrm{X})$ & + & USA & $\begin{array}{c}\text { Cruden } 1968 \text { as } \\
\text { A. u. occidentalis Walker, } 1908 \text { and } \\
\text { A. u. umbrosa Walker, } 1908\end{array}$ \\
\hline 16. & A. verticalis Hagen, 1861 & $27(26 \mathrm{~A}+\mathrm{X})$ & + & USA & Hung 1971 \\
\hline 17. & A. viridis Eversmann, 1836 & $26(24 \mathrm{~A}+$ neo-XY $)$ & + & Finland & Oksala 1943 \\
\hline & & $-n-$ & + & Russia & Perepelov et al. 1998 \\
\hline 18. & A. walkeri Kennedy, 1917 & $27(26 \mathrm{~A}+\mathrm{X})$ & + & USA & Cruden 1968 \\
\hline 19. & Anaciaeschna jaspidea (Burmeister, 1839) & $27(26 \mathrm{~A}+\mathrm{X})$ & + & India & Walia and Sandhu 1999 \\
\hline 20. & Anax amazili (Burmeister, 1839) & $27(26 \mathrm{~A}+\mathrm{X})$ & - & Argentina & Capitulo et al. 1991 \\
\hline & & $-»-$ & + & Argentina & Mola et al. 1999 \\
\hline 21. & A. concolor Brauer, 1865 & $27(26 \mathrm{~A}+\mathrm{X})$ & + & Surinam & Kiauta 1979a \\
\hline 22. & A. ephippiger (Burmeister, 1839) & $13(12 \mathrm{~A}+\mathrm{X})$ & + & India & $\begin{array}{c}\text { Seshachar and Bagga } 1962 \\
\text { as Hemianax ephippiger } \\
\text { (Burmeister, 1839) }\end{array}$ \\
\hline & & $14(12 \mathrm{~A}+$ neo-XY $)$ & + & India & Kiauta 1969 a as H. ephippiger \\
\hline
\end{tabular}




\begin{tabular}{|c|c|c|c|c|c|}
\hline \multicolumn{2}{|c|}{ Taxon } & \multirow{2}{*}{$\begin{array}{c}\begin{array}{c}\text { Karyotype } \\
\text { formula } \mathbf{2 n}\end{array} \\
15(14 \mathrm{~A}+\mathrm{X}) \\
\end{array}$} & \multirow{2}{*}{$\begin{array}{c}\begin{array}{c}\text { m-chromo } \\
\text { somes }\end{array} \\
+ \\
\end{array}$} & \multirow{2}{*}{$\begin{array}{c}\text { Country } \\
\text { Nepal }\end{array}$} & \multirow{2}{*}{$\begin{array}{c}\text { References } \\
\text { Kiauta and Kiauta } 1982\end{array}$} \\
\hline 23. & A. guttatus (Burmeister, 1839) & & & & \\
\hline \multirow[t]{2}{*}{24.} & \multirow[t]{2}{*}{ A. immaculiformis Rambur, 1842} & $27(26 \mathrm{~A}+\mathrm{X})$ & + & India & Sangal and Tyagi 1982 \\
\hline & & $-»-$ & + & India & Walia et al. 2018 \\
\hline \multirow[t]{3}{*}{25.} & \multirow[t]{3}{*}{ A. imperator Leach, 1815} & $27(26 \mathrm{~A}+\mathrm{X})$ & + & France & Kiauta 1965,1969 a \\
\hline & & $-»-$ & - & Kenya & Wasschner 1985 \\
\hline & & $-»-$ & + & Russia & Perepelov and Bugrov 2002 \\
\hline \multirow[t]{5}{*}{26.} & \multirow[t]{5}{*}{ A. junius (Drury, 1773) } & $27(26 \mathrm{~A}+\mathrm{X})$ & + & USA & McGill 1904, 1907 \\
\hline & & $-»-$ & + & USA & Lefevre and McGill 1908 \\
\hline & & $-n-$ & - & Japan & Kichijo 1942a \\
\hline & & $-n-$ & + & \multirow[t]{2}{*}{ USA } & \multirow[t]{2}{*}{ Cruden 1968} \\
\hline & & $-n-$ & - & & \\
\hline 27. & A. longipes Hagen, 1861 & $27(26 \mathrm{~A}+\mathrm{X})$ & + & USA & Cruden 1968 \\
\hline \multirow[t]{4}{*}{28.} & \multirow[t]{4}{*}{ A. nigrofasciatus Oguma, 1915} & $27(26 \mathrm{~A}+\mathrm{X})$ & + & Nepal & $\begin{array}{c}\text { Kiauta 1974, } 1975 \\
\text { (A. n. nigrolineatus Fraser, 1935) } \\
\end{array}$ \\
\hline & & $25(24 \mathrm{~A}+\mathrm{X})$ & + & India & $\begin{array}{c}\text { Sandhu and Malhotra 1994a } \\
\text { (A. n. nigrolineatus })\end{array}$ \\
\hline & & $27(26 A+X)$ & + & India & $\begin{array}{l}\text { Walia and Sandhu } 1999 \\
\text { (A.n.nigrolineatus })\end{array}$ \\
\hline & & $-»-$ & + & India & Walia et al. 2018 (A.n. nigrolineatus) \\
\hline 29. & A. papuensis (Burmeister, 1839) & $27(26 \mathrm{~A}+\mathrm{X})$ & + & Australia & $\begin{array}{l}\text { Kiauta 1968c, 1969a as Hemianax } \\
\text { papuensis (Burmeister, 1839) }\end{array}$ \\
\hline \multirow[t]{5}{*}{30.} & \multirow[t]{5}{*}{ A. parthenope (Selys, 1839) } & $27(26 \mathrm{~A}+\mathrm{X})$ & + & Japan & $\begin{array}{c}\text { Omura } 1957 \text { as } A \text {. parthenope julius } \\
\text { Brauer, } 1865\end{array}$ \\
\hline & & $-n-$ & + & India & Thomas and Prasad 1986 \\
\hline & & $-»-$ & + & China & Zhu and Wu 1986 as A. p. julius \\
\hline & & $25(24 \mathrm{~A}+\mathrm{X})$ & + & Japan & Suzuki and Saitoh 1990 as $A . p$. julius \\
\hline & & $27(26 \mathrm{~A}+\mathrm{X})$ & + & India & Sandhu and Malhotra 1994a \\
\hline 31. & Andaeschna unicolor (Martin, 1908) & $27(26 \mathrm{~A}+\mathrm{X})$ & + & Bolivia & $\begin{array}{c}\text { Cumming } 1964 \text { as Aeshna cf. unicolor } \\
\text { Martin, } 1908\end{array}$ \\
\hline 32. & Austroaeschna anacantha Tillyard, 1908 & $27(26 \mathrm{~A}+\mathrm{X})$ & + & Australia & $\begin{array}{l}\text { Kiauta } 1968 \mathrm{c} \text { as Acanthaeschna } \\
\text { anacantha (Tillyard, 1908) }\end{array}$ \\
\hline 33. & A. multipunctata (Martin, 1901) & $27(26 \mathrm{~A}+\mathrm{X})$ & + & Australia & $\begin{array}{l}\text { Kiauta } 1968 \mathrm{c} \text { as Acanthaeschna } \\
\text { multipunctata (Martin, 1901) }\end{array}$ \\
\hline 34. & Basiaeschna janata (Say, 1939) & $25(24 \mathrm{~A}+\mathrm{X})$ & - & USA & Cruden 1968 \\
\hline 35. & Boyeria maclachlani (Selys, 1883) & $27(26 \mathrm{~A}+\mathrm{X})$ & + & Japan & Omura 1957 \\
\hline 36. & B. vinosa (Say, 1839) & $27(26 \mathrm{~A}+\mathrm{X})$ & - & USA & Cruden 1968 \\
\hline 37. & \begin{tabular}{|l} 
Caliaeschna microstigma (Schneider, \\
1845 )
\end{tabular} & $16(14 \mathrm{~A}+$ neo-XY $)$ & + & Greece & Kiauta 1972a \\
\hline 38. & Castoraeschna castor (Brauer, 1865) & $27(26 \mathrm{~A}+\mathrm{X})$ & + & Brazil & Kiauta $1972 \mathrm{~b}$ \\
\hline 39. & Cephalaeschna orbifrons Selys, 1883 & $25(24 \mathrm{~A}+\mathrm{X})$ & + & Nepal & Kiauta 1975 \\
\hline 40. & Cephalaeschna sp. & $25(24 \mathrm{~A}+\mathrm{X})$ & + & India & Sandhu and Malhotra 1994a \\
\hline 41. & Coryphaeschna adnexa (Hagen, 1961) & $27(26 \mathrm{~A}+\mathrm{X})$ & - & Bolivia & Cumming 1964 \\
\hline \multirow[t]{3}{*}{42.} & \multirow[t]{3}{*}{ C. perrensi (McLachlan, 1887) } & $25(24 \mathrm{~A}+\mathrm{X})$ & - & Argentina & Capitulo et al. 1991 \\
\hline & & $27(26 \mathrm{~A}+\mathrm{X})$ & + & Argentina & Mola et al. 1999 \\
\hline & & $-n-$ & + & Argentina & De Gennaro et al. 2008 \\
\hline 43. & C. viriditas Calvert, 1952 & $23(22 \mathrm{~A}+\mathrm{X})$ & + & Surinam & Kiauta $1979 \mathrm{a}$ \\
\hline \multirow[t]{2}{*}{44.} & \multirow[t]{2}{*}{ Gynacantha bayadera Selys, 1891} & $25(24 \mathrm{~A}+\mathrm{X})$ & + & India & Walia 2007 as \\
\hline & & $27(26 \mathrm{~A}+\mathrm{X})$ & + & & G. milliardi Fraser, 1936 \\
\hline 45. & G. hyalina Selys, 1882 & $28(26 \mathrm{~A}+\mathrm{XX})^{*}$ & + & India & Tyagi $1978 \mathrm{a}, \mathrm{b}$ \\
\hline 46. & G. interioris Williamson, 1923 & $26(24 \mathrm{~A}+$ neo-XY $)$ & + & Surinam & Kiauta $1979 \mathrm{a}$ \\
\hline & & $-»-$ & + & Brazil & Ferreira et al. 1979 \\
\hline 47. & G. japonica Bartenev, 1909 & $27(26 \mathrm{~A}+\mathrm{X})$ & + & Japan & Omura 1957 \\
\hline 48. & $\begin{array}{l}\text { Gynacanthaeschna sikkima (Karsch, } \\
\text { 1891) }\end{array}$ & $27(26 \mathrm{~A}+\mathrm{X})$ & + & India & Walia et al. 2016 \\
\hline 49. & Oplonaeschna armata (Hagen, 1861) & $27(26 \mathrm{~A}+\mathrm{X})$ & + & Mexico & Kiauta 1970a \\
\hline 50. & Planaeschna milnei (Selys, 1883) & $27(26 \mathrm{~A}+\mathrm{X})$ & + & Japan & Kiauta $1968 c, 1969 a$ \\
\hline 51. & Remartinia luteipennis (Burmeister, 1839) & $25(24 \mathrm{~A}+\mathrm{X})$ & + & Surinam & $\begin{array}{c}\text { Kiauta } 1979 \text { as Coryphaeschna l. } \\
\text { luteipennis Burmeister, } 1839\end{array}$ \\
\hline & & $27(26 \mathrm{~A}+\mathrm{X})$ & + & Brazil & Ferreira et al. 1979 as C. l. luteipennis \\
\hline 52. & Rhionaeschna bonariensis (Rambur, 1842) & $26(24 \mathrm{~A}+$ neo-XY) & + & $\begin{array}{l}\text { Argentina, } \\
\text { Uruguay }\end{array}$ & $\begin{array}{c}\text { Mola and Papeschi } 1994 \text { as Aeschna } \\
\text { bonariensis Rambur, } 1842\end{array}$ \\
\hline
\end{tabular}




\begin{tabular}{|c|c|c|c|c|c|}
\hline \multicolumn{2}{|c|}{ Taxon } & \multirow{2}{*}{ 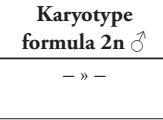 } & \multirow{2}{*}{$\begin{array}{c}\begin{array}{c}\text { m-chromo } \\
\text { somes }\end{array} \\
+\end{array}$} & \multirow{2}{*}{$\begin{array}{c}\text { Country } \\
\text { Argentina, } \\
\text { Uruguay }\end{array}$} & \multirow{2}{*}{$\begin{array}{c}\text { References } \\
\text { Mola } 1995 \text { as } A \text {. bonariensis }\end{array}$} \\
\hline 52. & Rhionaeschna bonariensis (Rambur, 1842) & & & & \\
\hline 53. & Rh. californica (Calvert, 1895) & $27(26 \mathrm{~A}+\mathrm{X})$ & + & Canada & $\begin{array}{c}\text { Kiauta 1973a as } \\
\text { Aeshna californica Calvert, } 1895\end{array}$ \\
\hline \multirow[t]{2}{*}{54.} & \multirow[t]{2}{*}{ Rh. confusa (Rambur, 1842) } & $27(26 \mathrm{~A}+\mathrm{X})$ & + & $\begin{array}{c}\text { Argentina, } \\
\text { Uruguay }\end{array}$ & $\begin{array}{l}\text { Mola and Papeschi } 1994 \text { as } \\
\text { Aeshna confuse Rambur, } 1842\end{array}$ \\
\hline & & $-"-$ & + & $\begin{array}{c}\text { Argentina, } \\
\text { Uruguay }\end{array}$ & Mola 1995 as $A$. confuse \\
\hline 55. & Rh. diffinis (Rambur, 1842) & $21(20 A+X)$ & + & Bolivia & $\begin{array}{c}\text { Cumming } 1964 \text { as } \\
\text { Aeshna d. diffinis Rambur, } 1842\end{array}$ \\
\hline 56. & Rh. intricata (Martin, 1908) & $19(18 \mathrm{~A}+\mathrm{X})$ & + & Bolivia & $\begin{array}{c}\text { Cumming } 1964 \text { as } \\
\text { Aeshna intricata Martin, } 1908\end{array}$ \\
\hline 57. & Rh. peralta (Ris, 1918) & $27(26 A+X)$ & + & Bolivia & $\begin{array}{c}\text { Cumming } 1964 \text { as Aeshna peralta } \\
\text { Ris, } 1918\end{array}$ \\
\hline \multirow[t]{2}{*}{58.} & \multirow[t]{2}{*}{ Rh. planaltica (Calvert, 1845) } & $16(14 \mathrm{~A}+$ neo-XY $)$ & + & Argentina & $\begin{array}{l}\text { Mola and Papeschi } 1994 \text { as Aeschna } \\
\text { cornigera planaltica Calvert, } 1952\end{array}$ \\
\hline & & $-n-$ & + & Argentina & Mola 1995 as A. c. planaltica \\
\hline 59. & $\begin{array}{l}\text { Staurophlebia reticulata } \\
\text { (Burmeister, 1839) }\end{array}$ & $27(26 \mathrm{~A}+\mathrm{X})$ & + & Brazil & $\begin{array}{c}\text { Souza Bueno } 1982(\text { S. } r \text { r. reticulata } \\
\text { (Burmeister, 1839)) }\end{array}$ \\
\hline
\end{tabular}

Petaluroidea

Petaluridae

\begin{tabular}{l|l|c|c|c|c}
\hline 60. & Tachopteryx thoreyi $($ Hagen, 1857) & $19(18 \mathrm{~A}+\mathrm{X})$ & + & USA & Cumming 1964 \\
\hline 61. & Tanypteryx hageni $($ Selys, 1879) & $17(16 \mathrm{~A}+\mathrm{X})$ & + & USA & Cruden 1968 \\
\hline 62. & T. pryeri $($ Selys, 1889) & $17(16 \mathrm{~A}+\mathrm{X})$ & + & Japan & Kichijo 1939, 1942a \\
\hline \multirow{2}{*}{63.} & \multirow{2}{*}{ Uropetala carovei (White, 1846) } & $17(16 \mathrm{~A}+\mathrm{X})^{* *}$ & + & New Zealand & Wolfe 1953 \\
\cline { 3 - 5 } & & $25(24 \mathrm{~A}+\mathrm{X})$ & + & New Zealand & Jensen and Mahanty 1978 \\
\cline { 3 - 5 } & & $-»-$ & + & New Zealand & Jensen 1980 \\
\hline
\end{tabular}

GoMPHOIDEA

Gomphidae

\begin{tabular}{|c|c|c|c|c|c|}
\hline \multirow[t]{2}{*}{64.} & \multirow[t]{2}{*}{ Anisogomphus bivittatus (Selys, 1854) } & $23(22 \mathrm{~A}+\mathrm{X})$ & + & India & Das 1956 \\
\hline & & $-»-$ & + & India & Walia and Chahal 2020 \\
\hline 65. & A. occipitalis (Selys, 1854) & $23(22 \mathrm{~A}+\mathrm{X})$ & - & Nepal & Kiauta 1974,1975 \\
\hline 66. & Aphylla edentata Selys, 1869 & $23(22 \mathrm{~A}+\mathrm{X})$ & - & Bolivia & Cumming 1964 \\
\hline 67. & A. producta Selys, 1854 & $23(22 \mathrm{~A}+\mathrm{X})$ & - & Bolivia & Cumming 1964 \\
\hline \multirow[t]{2}{*}{68.} & \multirow[t]{2}{*}{ A. theodorina (Navas, 1933) } & $23(22 \mathrm{~A}+\mathrm{X})$ & + & Surinam & Kiauta 1979a \\
\hline & & $-n-$ & + & Brazil & Ferreira et al. 1979 \\
\hline 69. & A. williamsoni (Gloyd, 1936) & $23(22 \mathrm{~A}+\mathrm{X})$ & + & USA & Kiauta and Brink 1978 \\
\hline 70. & Aphylla sp. & $23(22 \mathrm{~A}+\mathrm{X})$ & + & Argentina & Mola 2007 \\
\hline 71. & Arigomphus lentulus (Needham, 1902) & $23(22 \mathrm{~A}+\mathrm{X})$ & - & USA & $\begin{array}{c}\text { Cruden } 1968 \text { as Gomphus lentulus } \\
\text { Needham, } 1902\end{array}$ \\
\hline 72. & A. pallidus (Rambur, 1842) & $23(22 \mathrm{~A}+\mathrm{X})$ & - & USA & $\begin{array}{c}\text { Cumming } 1964 \text { as Gomphus pallidus } \\
\text { Rambur, } 1842\end{array}$ \\
\hline 73. & A. submedianus (Williamson, 1914) & $23(22 \mathrm{~A}+\mathrm{X})$ & - & USA & $\begin{array}{c}\text { Cruden } 1968 \text { as Gomphus } \\
\text { submedianus Williamson, } 1914\end{array}$ \\
\hline \multirow[t]{3}{*}{74.} & \multirow[t]{3}{*}{ Asiagomphus melaenops (Selys, 1854) } & $23(22 A+X)$ & + & Japan & $\begin{array}{l}\text { Toyoshima and Hirai } 1953 \text { as } \\
\text { Gomphus melaenops Selys, } 1854\end{array}$ \\
\hline & & $-»-$ & + & Japan & Hirai 1956 as G. melaenops \\
\hline & & $-»-$ & + & USA & Cruden 1968 as G. melaenops \\
\hline 75. & Burmagomphus pyramidalis Laidlaw, 1922 & $23(22 \mathrm{~A}+\mathrm{X})$ & + & India & Tyagi 1977 \\
\hline 76. & Davidius nanus (Selys, 1869) & $23(22 \mathrm{~A}+\mathrm{X})$ & - & Japan & Kichijo 1939, 1942a \\
\hline 77. & Dromogomphus spinosus (Selys, 1854) & $23(22 \mathrm{~A}+\mathrm{X})$ & + & USA & Cruden 1968 \\
\hline 78. & D. spoliatus (Hagen, 1857) & $23(22 \mathrm{~A}+\mathrm{X})$ & + & USA & Cruden 1968 \\
\hline 79. & Epigomphus llama Calvert, 1903 & $23(22 \mathrm{~A}+\mathrm{X})$ & - & Bolivia & Cumming 1964 \\
\hline 80. & Erpetogomphus designatus Hagen, 1857 & $23(22 \mathrm{~A}+\mathrm{X})$ & + & USA & Cumming 1964 \\
\hline 81. & E. diadophis Calvert, 1905 & $23(22 \mathrm{~A}+\mathrm{X})$ & - & USA & Cumming 1964 \\
\hline 82. & E. ophibolus Calvert, 1905 & $23(22 \mathrm{~A}+\mathrm{X})$ & + & Mexico & Kiauta 1970a \\
\hline 83. & Gomphoides sp. & $23(22 \mathrm{~A}+\mathrm{X})$ & - & Bolivia & Cumming 1964 \\
\hline 84. & Gomphus confraternus Selys, 1873 & $23(22 \mathrm{~A}+\mathrm{X})$ & + & USA & Cruden 1968 \\
\hline \multirow[t]{2}{*}{85.} & \multirow[t]{2}{*}{ G. exilis Selys, 1854} & $23(22 \mathrm{~A}+\mathrm{X})$ & + & USA & Cruden 1968 \\
\hline & & $-n-$ & + & Canada & Kiauta 1969a \\
\hline 86. & G. graslini Rambur, 1842 & $\begin{array}{l}\text { 12(10A+neo-neo- } \\
\text { XY) }\end{array}$ & + & France & Kiauta 1968d, 1969a \\
\hline
\end{tabular}




\begin{tabular}{|c|c|c|c|c|c|}
\hline \multicolumn{2}{|c|}{ Taxon } & \multirow{2}{*}{$\begin{array}{c}\begin{array}{c}\text { Karyotype } \\
\text { formula } \mathbf{2 n} \delta\end{array} \\
23(22 \mathrm{~A}+\mathrm{X})\end{array}$} & \multirow{2}{*}{\begin{tabular}{c|}
$\begin{array}{c}\text { m-chromo } \\
\text { somes }\end{array}$ \\
+
\end{tabular}} & \multirow{2}{*}{$\begin{array}{c}\text { Country } \\
\text { France } \\
\end{array}$} & \multirow{2}{*}{$\begin{array}{c}\text { References } \\
\text { Kiauta } 1973 \mathrm{~b} \\
\end{array}$} \\
\hline 87. & G. pulchellus Selys, 1840 & & & & \\
\hline 88. & G. vulgatissimus (Linnaeus, 1758) & $23(22 \mathrm{~A}+\mathrm{X})$ & - & Russia & Perepelov et al. 2001 \\
\hline \multirow[t]{5}{*}{89.} & \multirow[t]{5}{*}{ Ictinogomphus rapax (Rambur, 1942) } & $23(22 \mathrm{~A}+\mathrm{X})$ & + & India & Asana and Makino 1935 \\
\hline & & $-»-$ & + & India & Makino 1935 \\
\hline & & $-»-$ & + & India & Kichijo 1942a \\
\hline & & $-n-$ & + & India & Omura $1949,1952,1953$ \\
\hline & & $-»-$ & + & India & Dasgupta 1957 \\
\hline \multirow[t]{2}{*}{90.} & \multirow[t]{2}{*}{ Nepogomphus modestus (Selys, 1878) } & $23(22 \mathrm{~A}+\mathrm{X})$ & - & India & Walia et al. 2006 \\
\hline & & $-»-$ & - & India & Walia and Chahal 2014 \\
\hline 91. & Nihonogomphus ruptus (Selys, 1858) & $23(22 \mathrm{~A}+\mathrm{X})$ & - & Russia & Perepelov et al. 2001 \\
\hline 92. & N. viridis Oguma, 1926 & $23(22 A+X)$ & + & Japan & Omura 1957 \\
\hline 93. & Nychogomphus duaricus (Fraser, 1924) & $22(20 \mathrm{~A}+$ neo-XY $)$ & + & India & Tyagi 1977 \\
\hline 94. & Octogomphus specularis (Hagen, 1859) & $23(22 \mathrm{~A}+\mathrm{X})$ & - & USA & Cruden 1968 \\
\hline \multirow[t]{3}{*}{95.} & \multirow{3}{*}{$\begin{array}{l}\text { Onychogomphus forcipatus } \\
\text { (Linnaeus, 1758) }\end{array}$} & $25(24 \mathrm{~A}+\mathrm{X})$ & - & Finland & Oksala 1945 \\
\hline & & $22(20 \mathrm{~A}+$ neo-XY $)$ & - & \multirow{2}{*}{ Austria } & \multirow{2}{*}{ Kiauta 1969a } \\
\hline & & $25(24 \mathrm{~A}+\mathrm{X})$ & - & & \\
\hline 96. & O. saundersii Selys, 1854 & $22(20 \mathrm{~A}+$ neo-XY $)$ & + & India & $\begin{array}{c}\text { Tyagi } 1977 \\
\text { (O. s. duaricus Fraser, 1924) }\end{array}$ \\
\hline \multirow[t]{2}{*}{97.} & \multirow[t]{2}{*}{ Ophiogomphus bison Selys, 1873} & $23(22 \mathrm{~A}+\mathrm{X})$ & - & \multirow[t]{2}{*}{ USA } & \multirow[t]{2}{*}{ Cruden 1968} \\
\hline & & $25(24 \mathrm{~A}+\mathrm{X})$ & - & & \\
\hline \multirow[t]{3}{*}{98.} & \multirow[t]{3}{*}{ O. cecilia (Fourcroy, 1785) } & $24(22 \mathrm{~A}+\mathrm{XX})^{*}$ & - & Finland & Oksala 1945 \\
\hline & & $23(22 \mathrm{~A}+\mathrm{X})$ & - & Russia & Perepelov et al. 1998 \\
\hline & & $-n-$ & - & Russia & Perepelov and Bugrov 2001a \\
\hline 99. & O. colubrinus Selys, 1854 & $23(22 \mathrm{~A}+\mathrm{X})$ & - & USA & Cruden 1968 \\
\hline 100. & O. obscurus Bartenev, 1909 & $23(22 \mathrm{~A}+\mathrm{X})$ & - & Russia & Perepelov and Bugrov 2001b \\
\hline 101. & O. occidentalis Hagen, 1882 & $23(22 \mathrm{~A}+\mathrm{X})$ & - & USA & Cruden 1968 \\
\hline 102. & O. rupinsulensis (Walsh, 1862) & $23(22 \mathrm{~A}+\mathrm{X})$ & - & USA & Cruden 1968 \\
\hline 103. & Phanogomphus lividus (Selys, 1854) & $23(22 \mathrm{~A}+\mathrm{X})$ & + & USA & $\begin{array}{c}\text { Cruden } 1968 \text { as } \\
\text { Gomphus lividus Selys, } 1854 \\
\end{array}$ \\
\hline 104. & Ph. militaris (Hagen, 1858) & $23(22 \mathrm{~A}+\mathrm{X})$ & - & USA & $\begin{array}{c}\text { Cruden } 1968 \text { as } \\
\text { Gomphus militaris Hagen, } 1858\end{array}$ \\
\hline 105. & Ph. spicatus (Selys, 1854) & $23(22 \mathrm{~A}+\mathrm{X})$ & + & USA & $\begin{array}{c}\text { Cruden } 1968 \text { as } \\
\text { Gomphus spicatus Selys, } 1854\end{array}$ \\
\hline \multirow[t]{2}{*}{106.} & \multirow[t]{2}{*}{ Paragomphus lineatus (Selys, 1850) } & $23(22 \mathrm{~A}+\mathrm{X})$ & - & Nepal & Kiauta 1974,1975 \\
\hline & & $-n-$ & - & India & Walia and Chahal 2014 \\
\hline 107. & P. capricornis (Förster, 1914) & $23(22 \mathrm{~A}+\mathrm{X})$ & - & Thailand & Kiauta and Kiauta 1983 \\
\hline 108. & Phyllocycla propinqua Belle, 1972 & $21(20 \mathrm{~A}+\mathrm{X})$ & - & Argentina & De Gennaro 2004 \\
\hline 109. & Phyllocycla sp. & $23(22 \mathrm{~A}+\mathrm{X})$ & - & Bolivia & Cumming 1964 \\
\hline 110. & Phyllocycla sp. 1 & $23(22 \mathrm{~A}+\mathrm{X})$ & + & Argentina & Mola 2007 \\
\hline 111. & Phyllocycla sp. 2 & $23(22 \mathrm{~A}+\mathrm{X})$ & - & Argentina & Mola 2007 \\
\hline 112. & $\begin{array}{l}\text { Phyllogomphoides undulatus } \\
\text { (Needham, 1944) }\end{array}$ & $23(22 \mathrm{~A}+\mathrm{X})$ & + & Surinam & Kiauta 1979a \\
\hline 113. & Progomphus borealis McLachlan, 1873 & $23(22 \mathrm{~A}+\mathrm{X})$ & - & USA & Cruden 1968 \\
\hline 114. & P. intricatus (Hagen, 1857) & $23(22 \mathrm{~A}+\mathrm{X})$ & - & Bolivia & Cumming 1964 \\
\hline 115. & P. obscurus (Rambur, 1842) & $23(22 \mathrm{~A}+\mathrm{X})$ & - & USA & Cruden 1968 \\
\hline 116. & P. phyllochromus Ris, 1918 & $23(22 \mathrm{~A}+\mathrm{X})$ & + & Bolivia & Cumming 1964 \\
\hline 117. & Scalmogomphus bistrigatus (Hagen, 1854) & $23(22 \mathrm{~A}+\mathrm{X})$ & - & Nepal & $\begin{array}{c}\text { Kiauta 1974, } 1975 \text { as Onychogomphus } \\
\text { bistrigatus (Hagen, 1854) }\end{array}$ \\
\hline 118. & Shaogomphus postocularis (Selys, 1869) & $23(22 \mathrm{~A}+\mathrm{X})$ & + & Japan & $\begin{array}{c}\text { Omura } 1957 \text { as } \\
\text { Gomphus postocularis Selys, } 1869\end{array}$ \\
\hline & & $-"-$ & - & Russia & $\begin{array}{c}\text { Perepelov et al. } 2001 \text { as } \\
\text { Gomphus epophtalmus Selys, } 1872\end{array}$ \\
\hline 119. & Sieboldius albardae Selys, 1886 & $23(22 \mathrm{~A}+\mathrm{X})$ & + & Japan & Omura 1957 \\
\hline 120. & Stylogomphus suzukii (Matsumura, 1926) & $23(22 \mathrm{~A}+\mathrm{X})$ & + & Japan & Oguma 1930 \\
\hline & & $-n-$ & + & Japan & Kichijo 1942a \\
\hline 121. & Stylurus flavipes (Charpentier, 1825) & $23(22 \mathrm{~A}+\mathrm{X})$ & + & Russia & Perepelov and Bugrov 2001b \\
\hline 122. & S. plagiatus (Selys, 1854) & $23(22 \mathrm{~A}+\mathrm{X})$ & + & USA & $\begin{array}{c}\text { Cruden } 1968 \text { as } \\
\text { Gomphus plagiatus Selys, } 1854\end{array}$ \\
\hline 123. & S. scudderi (Selys, 1873) & $23(22 \mathrm{~A}+\mathrm{X})$ & - & USA & $\begin{array}{c}\text { Cruden } 1968 \text { as } \\
\text { Gomphus scudderi Selys, } 1873\end{array}$ \\
\hline
\end{tabular}




\begin{tabular}{|c|c|c|c|c|c|}
\hline \multicolumn{2}{|c|}{ Taxon } & \multirow{2}{*}{$\begin{array}{c}\begin{array}{c}\text { Karyotype } \\
\text { formula } 2 \mathbf{n}\end{array} \\
22(20 \mathrm{~A}+\text { neo-XY })\end{array}$} & \multirow{2}{*}{$\begin{array}{c}\begin{array}{c}\text { m-chromo } \\
\text { somes }\end{array} \\
-\end{array}$} & \multirow{2}{*}{$\begin{array}{c}\text { Country } \\
\text { USA }\end{array}$} & \multirow{2}{*}{$\begin{array}{c}\text { References } \\
\text { Kiauta and Brink } 1978 \text { as } \\
\text { Gomphus townesi Gloyd, } 1936\end{array}$} \\
\hline 124. & S. townesi Gloyd, 1936 & & & & \\
\hline 125. & Temnogomphus bivittatus (Selys, 1854) & $23(22 \mathrm{~A}+\mathrm{X})$ & + & Nepal & Kiauta 1975 \\
\hline \multirow[t]{2}{*}{126.} & \multirow[t]{2}{*}{ Trigomphus citimus (Needham, 1931) } & $21(20 A+X)$ & + & Japan & $\begin{array}{l}\text { Toyoshima and Hirai } 1953 \\
\text { (T. c. tabei Asahina, 1949) }\end{array}$ \\
\hline & & $-"-$ & + & Japan & Hirai 1956 (T.c. tabei) \\
\hline \multirow[t]{4}{*}{127.} & \multirow[t]{4}{*}{ T. interruptus (Selys, 1854) } & $19(18 \mathrm{~A}+\mathrm{X})$ & + & Japan & Oguma 1930 \\
\hline & & $-»-$ & + & Japan & Toyoshima and Hirai 1953 \\
\hline & & $-»-$ & + & Japan & Hirai 1956 \\
\hline & & $-»-$ & + & Japan & Omura 1957 \\
\hline 128. & T. melampus (Selys, 1869) & $21(20 \mathrm{~A}+\mathrm{X})$ & - & Japan & $\begin{array}{c}\text { Oguma 1930, } 1942 \text { as T. unifasciatus } \\
\text { (Oguma 1926) }\end{array}$ \\
\hline 129. & Zonophora callipus Selys, 1869 & $23(22 \mathrm{~A}+\mathrm{X})$ & + & Surinam & Kiauta $1979 \mathrm{a}$ \\
\hline \multicolumn{6}{|c|}{ LIBELLULOIDEA } \\
\hline \multicolumn{6}{|c|}{ Macromiidae } \\
\hline 130. & Didymops transversa (Say, 1839) & $25(24 \mathrm{~A}+\mathrm{X})$ & + & USA & Cruden 1968 \\
\hline 131. & Epophthalmia frontalis (Selys, 1871) & $25(24 \mathrm{~A}+\mathrm{X})$ & + & India & $\begin{array}{c}\text { Dasgupta } 1957 \\
\text { (E.f. frontalis (Selys, 1871)) }\end{array}$ \\
\hline 132. & Macromia daimoji Okumura, 1949 & $25(24 \mathrm{~A}+\mathrm{X})$ & - & Japan & Katatani 1987 \\
\hline 133. & M. amphigenia Selys, 1871 & $25(24 \mathrm{~A}+\mathrm{X})$ & - & Russia & $\begin{array}{l}\text { Perepelov and Bugrov 2001b } \\
\text { (M. a. fraenata Martin, 1906) }\end{array}$ \\
\hline \multirow[t]{2}{*}{134.} & \multirow[t]{2}{*}{ M. magnifica (McLachlan, 1874) } & $25(24 A+X)$ & + & \multirow[t]{2}{*}{ USA } & \multirow[t]{2}{*}{ Cruden 1968} \\
\hline & & $-n-$ & - & & \\
\hline \multirow[t]{2}{*}{135.} & \multirow[t]{2}{*}{ M. moorei Selys, 1874} & $25(24 \mathrm{~A}+\mathrm{X})$ & + & Nepal & Kiauta 1977 \\
\hline & & $-»-$ & + & India & Walia and Chahal 2018 \\
\hline \multicolumn{6}{|c|}{ Corduliidae } \\
\hline \multirow[t]{6}{*}{136.} & Cordulia aenea (Linnaeus, 1758) & $25(24 \mathrm{~A}+\mathrm{X})$ & - & Finland & Oksala 1939a \\
\hline & & $-»-$ & - & Former USSR & Makalowskaja 1940 \\
\hline & & $-n-$ & - & Netherlands & Kiauta 1968b, 1969a \\
\hline & & $-»-$ & - & Russia & Perepelov et al. 1998 \\
\hline & & $-"-$ & - & Bulgaria & Grozeva and Marinov 2007 \\
\hline & & $-»-$ & - & Russia & Kuznetsova et al. 2018 \\
\hline 137. & C. shurtleffi Scudder, 1866 & $25(24 \mathrm{~A}+\mathrm{X})$ & + & USA & Cruden 1968 \\
\hline & & $-n-$ & + & Canada & Kiauta 1973a \\
\hline 138. & Dorocordulia libera (Selys, 1871) & $11(10 \mathrm{~A}+\mathrm{X})$ & - & USA & Cruden 1968 \\
\hline & & $13(12 \mathrm{~A}+\mathrm{X})$ & - & & \\
\hline & & $14(12 \mathrm{~A}+$ neo-XY) & - & USA & Kiauta 1969a \\
\hline & & $13(12 \mathrm{~A}+\mathrm{X})$ & - & & \\
\hline 139. & Epicordulia princeps (Hagen, 1861) & $25(24 \mathrm{~A}+\mathrm{X})$ & + & USA & Hung 1971 \\
\hline 140. & Epitheca bimaculata (Charpentier, 1825) & $25(24 \mathrm{~A}+\mathrm{X})$ & - & Russia & Perepelov 2003 \\
\hline & & $-»-$ & - & Russia & Kuznetsova et al. 2018 \\
\hline 141. & E. canis McLachlan, 1886 & $25(24 \mathrm{~A}+\mathrm{X})$ & + & USA & Cruden 1968 \\
\hline 142. & E. cynosura $($ Say, 1839) & $19(18 \mathrm{~A}+\mathrm{X})$ & - & USA & Cruden 1968 \\
\hline & & $21(20 \mathrm{~A}+\mathrm{X})$ & - & & \\
\hline 143. & E. petechialis (Muttkowski, 1911) & $21(20 A+X)$ & - & USA & $\begin{array}{l}\text { Cumming } 1964 \text { as Tetragoneuria } \\
\text { petechialis Muttkowski, } 1911\end{array}$ \\
\hline 144. & E. semiaquea (Burmeister, 1839) & $25(24 \mathrm{~A}+\mathrm{X})$ & - & USA & Cruden 1968 \\
\hline 145. & E. spinigera (Selys, 1871) & $25(24 \mathrm{~A}+\mathrm{X})$ & + & USA & Cruden 1968 \\
\hline & & $27(26 \mathrm{~A}+\mathrm{X})$ & - & USA & $\begin{array}{l}\text { Hung } 1971 \text { as Tetragoneuria spinigera } \\
\text { (Selys, 1871) }\end{array}$ \\
\hline 146. & Procordulia grayi (Selys, 1871) & $25(24 \mathrm{~A}+\mathrm{X})$ & + & New Zealand & Jensen 1980 \\
\hline 147. & P. smithii (White, 1846) & $25(24 \mathrm{~A}+\mathrm{X})$ & + & New Zealand & Jensen 1980 \\
\hline 148. & Rialla villosa Rambur, 1842 & $25(24 \mathrm{~A}+\mathrm{X})$ & + & Argentina & De Gennaro 2004 \\
\hline 149. & Somatochlora alpestris (Selys, 1840) & $25(24 \mathrm{~A}+\mathrm{X})$ & - & Switzerland & Kiauta and Kiauta 1980a \\
\hline & & $27(26 \mathrm{~A}+\mathrm{X})$ & + & & \\
\hline 150. & S. arctica (Zetterstedt, 1840) & $25(24 \mathrm{~A}+\mathrm{X})$ & + & Russia & Perepelov 2003 \\
\hline 151. & S. borisi Marinov, 2001 & $20(18 \mathrm{~A}+\mathrm{XY})$ & - & Bulgaria & Grozeva and Marinov 2007 \\
\hline 152. & S. flavomaculata (Van der Linden, 1825) & $25(24 \mathrm{~A}+\mathrm{X})$ & - & Former USSR & Makalowskaja 1940 \\
\hline & & $-»-$ & - & Russia & Perepelov 2003 \\
\hline & & $-»-$ & + & Russia & Kuznetsova et al. 2020b \\
\hline
\end{tabular}




\begin{tabular}{|c|c|c|c|c|c|}
\hline \multicolumn{2}{|c|}{ Taxon } & \multirow{2}{*}{$\begin{array}{c}\begin{array}{c}\text { Karyotype } \\
\text { formula } \mathbf{2 n} \delta\end{array} \\
25(24 \mathrm{~A}+\mathrm{X}) \\
\end{array}$} & \multirow{2}{*}{$\begin{array}{c}\begin{array}{c}\text { m-chromo } \\
\text { somes }\end{array} \\
-\end{array}$} & \multirow{2}{*}{\begin{tabular}{|c|} 
Country \\
Russia \\
\end{tabular}} & \multirow{2}{*}{$\begin{array}{c}\text { References } \\
\text { Perepelov et al. } 2001\end{array}$} \\
\hline 153. & S. graeseri Selys, 1887 & & & & \\
\hline \multirow[t]{2}{*}{154.} & \multirow[t]{2}{*}{ S. meridionalis Nielsen, 1935} & $25(24 \mathrm{~A}+\mathrm{X})$ & - & Slovenia & Kiauta and Kiauta 1995 \\
\hline & & $-»-$ & - & Bulgaria & Grozeva and Marinov 2007 \\
\hline \multirow[t]{4}{*}{155.} & \multirow[t]{4}{*}{ S. metallica (Van der Linden, 1825) } & $26(24 \mathrm{~A}+\mathrm{XX})^{*}$ & - & Finland & Oksala 1945 \\
\hline & & $25(24 \mathrm{~A}+\mathrm{X})$ & - & Finland & Nokkala et al. 2002 \\
\hline & & $-n-$ & - & Finland & Grozeva and Marinov 2007 \\
\hline & & $-n-$ & - & Russia & Perepelov and Bugrov 2001b \\
\hline 156. & S. semicircularis (Selys, 1871) & $25(24 \mathrm{~A}+\mathrm{X})$ & - & USA & Cruden 1968 \\
\hline \multirow[t]{2}{*}{157.} & \multirow[t]{2}{*}{ S. uchidai Fürster, 1909} & $25(24 \mathrm{~A}+\mathrm{X})$ & + & Japan & Oguma 1915,1930 \\
\hline & & $-»-$ & + & Japan & Kichijo 1942b \\
\hline \multirow[t]{2}{*}{158.} & \multirow[t]{2}{*}{ S. viridiaenea (Uhler, 1858) } & $25(24 \mathrm{~A}+\mathrm{X})$ & - & Japan & Oguma 1915,1930 \\
\hline & & $-»-$ & - & Japan & Kichijo 1942b \\
\hline \multicolumn{6}{|c|}{ Libellulidae } \\
\hline \multirow[t]{4}{*}{159.} & \multirow[t]{4}{*}{ Acisoma panorpoides Rambur, 1842} & $25(24 \mathrm{~A}+\mathrm{X})$ & + & Bangladesh, India & $\begin{array}{c}\text { Dasgupta } 1957 \text { (A.p.panorpoides } \\
\text { Rambur, 1842) }\end{array}$ \\
\hline & & $-n-$ & + & Nepal & Kiauta 1975 (A.p.panorpoides) \\
\hline & & $-»-$ & + & Thailand & $\begin{array}{c}\text { Kiauta and Kiauta } 1983 \\
\text { (A.p.panorpoides) }\end{array}$ \\
\hline & & $-»-$ & + & India & Tyagi 1982 \\
\hline 160. & Aethriamanta brevipennis (Rambur, 1842) & $25(24 \mathrm{~A}+\mathrm{X})$ & + & India & Dasgupta 1957 \\
\hline 161. & Anatya guttata (Erichson, 1848) & $25(24 \mathrm{~A}+\mathrm{X})$ & - & Surinam & Kiauta 1979a \\
\hline 162. & Atoconeura biordinata Karsch, 1899 & $21(20 \mathrm{~A}+\mathrm{X})$ & + & Sudan & Wasscher 1985 \\
\hline \multirow[t]{4}{*}{163.} & \multirow[t]{4}{*}{ Brachydiplax chalybea Brauer, 1868} & $25(24 \mathrm{~A}+\mathrm{X})$ & + & India & Dasgupta 1957 \\
\hline & & $-»-$ & + & India & Taygi 1982 \\
\hline & & $-n-$ & + & Thailand & Kiauta and Kiauta 1983 \\
\hline & & $-n-$ & + & India & Prasad and Thomas 1992 \\
\hline 164. & B. farinosa Krueger, 1902 & $25(24 \mathrm{~A}+\mathrm{X})$ & + & India & Dasgupta 1957 \\
\hline & & $-n-$ & + & India & Taygi 1982 \\
\hline & & $-n-$ & - & Thailand & Kiauta and Kiauta 1983 \\
\hline 165. & B. sobrina (Rambur, 1842) & $25(24 \mathrm{~A}+\mathrm{X})$ & + & India & Ray Chaudhuri and Dasgupta 1949 \\
\hline & & $-»-$ & + & India & Taygi 1982 \\
\hline & & $-»-$ & + & Nepal & Kiauta and Kiauta 1982 \\
\hline 166. & Brachvmesia furcata (Hagen, 1861) & $25(24 \mathrm{~A}+\mathrm{X})$ & + & Surinam & Kiauta $1979 \mathrm{a}$ \\
\hline & & $-»-$ & + & Argentina & Agopian and Mola 1988 \\
\hline & & $-n-$ & - & Brazil & Ferreira et al. 1979 \\
\hline & & $-n-$ & - & Brazil & Souza Bueno 1982 \\
\hline 167. & B. gravida (Calvert, 1890) & $25(24 \mathrm{~A}+\mathrm{X})$ & + & USA & $\begin{array}{c}\text { Cruden } 1968 \text { as Cannacria gravida } \\
\text { (Calvert, 1890) }\end{array}$ \\
\hline 168. & B. herbida (Gundlach, 1889) & $25(24 \mathrm{~A}+\mathrm{X})$ & + & Jamaica & $\begin{array}{l}\text { Cumming } 1964 \text { as Cannacria herbida } \\
\text { (Gundlach, 1889) }\end{array}$ \\
\hline 169. & Brachythemis contaminata & $25(24 \mathrm{~A}+\mathrm{X})$ & + & India & Asana and Makino 1935 \\
\hline & (Fabricius, 1793) & $-»-$ & + & India & Makino 1935 \\
\hline & & $-n-$ & + & India & Kichijo 1942b \\
\hline & & $-n-$ & + & India & Dasgupta 1957 \\
\hline & & $-»-$ & + & Nepal & Kiauta 1975 \\
\hline & & $-"-$ & + & India & Tyagi 1982 \\
\hline & & $-»-$ & + & Thailand & Kiauta and Kiauta 1983 \\
\hline 170. & B. lacustris (Kirby, 1899) & $25(24 \mathrm{~A}+\mathrm{X})$ & + & Sudan & Wasscher 1985 \\
\hline 171. & Bradinopyga cornuta Ris, 1911 & $25(24 \mathrm{~A}+\mathrm{X})$ & + & $\begin{array}{c}\text { Republic of South } \\
\text { Africa }\end{array}$ & Boyes et al. 1980 \\
\hline 172. & B. geminata (Rambur, 1842) & $25(24 \mathrm{~A}+\mathrm{X})$ & + & India & Dasgupta 1957 \\
\hline & & $-»-$ & + & India & Tyagi 1982 \\
\hline 173. & Brechmorhoga mendax (Hagen, 1861) & $25(24 \mathrm{~A}+\mathrm{X})$ & + & USA & Cruden 1968 \\
\hline & & $-n-$ & - & & \\
\hline 174. & B. nubecula (Rambur, 1842) & $25(24 \mathrm{~A}+\mathrm{X})$ & + & Bolivia & Cumming 1964 \\
\hline 175. & B. pertinax (Hagen, 1861) & $25(24 \mathrm{~A}+\mathrm{X})$ & - & Bolivia & $\begin{array}{c}\text { Cumming 1964 } \\
\text { (B.p.peruviana } \mathrm{Ris}, 1913)\end{array}$ \\
\hline 176. & Cannaphila vibex (Hagen, 1861) & $25(24 \mathrm{~A}+\mathrm{X})$ & + & Bolivia & Cumming 1964 \\
\hline 177. & Celithemis amanda (Hagen, 1861) & $25(24 \mathrm{~A}+\mathrm{X})$ & + & USA & Kiauta and Brink 1978 \\
\hline 178. & C. elisa (Hagen, 1861) & $25(24 \mathrm{~A}+\mathrm{X})$ & + & USA & Cruden 1968 \\
\hline 179. & C. fasciata Kirby, 1889 & $25(24 \mathrm{~A}+\mathrm{X})$ & + & USA & Cruden 1968 \\
\hline
\end{tabular}




\begin{tabular}{|c|c|c|c|c|c|}
\hline \multicolumn{2}{|c|}{ Taxon } & \multirow{2}{*}{$\begin{array}{c}\begin{array}{c}\text { Karyotype } \\
\text { formula } \mathbf{2 n}\end{array} \\
25(24 \mathrm{~A}+\mathrm{X}) \\
\end{array}$} & \multirow{2}{*}{$\begin{array}{c}\begin{array}{c}\text { m-chromo } \\
\text { somes }\end{array} \\
+\end{array}$} & \multirow{2}{*}{$\begin{array}{c}\text { Country } \\
\text { USA }\end{array}$} & \multirow{2}{*}{$\begin{array}{c}\text { References } \\
\text { Kiauta and Brink } 1978 \\
\end{array}$} \\
\hline 180. & C. ornata (Rambur, 1842) & & & & \\
\hline \multirow[t]{6}{*}{181.} & \multirow[t]{6}{*}{ Crocothemis erythraea (Brulle, 1832) } & $25(24 \mathrm{~A}+\mathrm{X})$ & + & India & Dasgupta 1957 \\
\hline & & $-»-$ & + & Kenya & Kiauta $1969 b$ \\
\hline & & $-n-$ & + & Italy & Kiauta 1971a \\
\hline & & $-»-$ & + & India & Prasad and Thomas 1992 \\
\hline & & $-"-$ & + & $\begin{array}{c}\text { Republic of South } \\
\text { Africa }\end{array}$ & Boyes et al. 1980 \\
\hline & & $-n-$ & + & India & Tyagi 1982 \\
\hline 182. & C. sanguinolenta (Burmeister, 1839) & $25(24 \mathrm{~A}+\mathrm{X})$ & + & $\begin{array}{c}\text { Kingdom of } \\
\text { Eswatini (Former } \\
\text { Swaziland) }\end{array}$ & Boyes et al. 1980 \\
\hline \multirow[t]{16}{*}{183.} & \multirow[t]{16}{*}{ C. servilia (Drury, 1773) } & $25(24 \mathrm{~A}+\mathrm{X})$ & + & India & Asana and Makino 1935 \\
\hline & & $-n-$ & + & India & Makino 1935 \\
\hline & & $-»-$ & + & India & Kichijo 1942b \\
\hline & & $-n-$ & + & India & Ray Chaudhuri and Dasgupta 1949 \\
\hline & & $-n-$ & + & Nepal & Kiauta 1975 \\
\hline & & $-»-$ & + & Philippines & Kiauta and Kiauta 1980b \\
\hline & & $-"-$ & + & Nepal & Kiauta and Kiauta 1982 \\
\hline & & $-»-$ & + & India & Tyagi 1982 \\
\hline & & $-n-$ & + & Thailand & Kiauta and Kiauta 1983 \\
\hline & & $-n-$ & + & Japan & Katatani 1987 \\
\hline & & $-n-$ & + & Japan & Higashi and Kayano 1993 \\
\hline & & $-n-$ & + & Japan, Taiwan & Higashi et al. 2001 \\
\hline & & $24(22 \mathrm{~A}+$ neo-XY $)$ & + & Japan & $\begin{array}{c}\text { Omura } 1955 \\
\text { (C. s. mariannae Kiauta, 983) }\end{array}$ \\
\hline & & $-"-$ & - & Japan & Kiauta 1983 (C. s. mariannae) \\
\hline & & $-n-$ & - & Japan & Katatani 1987 (C. s. mariannae) \\
\hline & & $-n-$ & - & Japan & Higashi et al. 2001 (C. s. mariannae) \\
\hline 184. & Dasythemis esmeralda Ris, 1910 & $25(24 \mathrm{~A}+\mathrm{X})$ & + & Bolivia & Cumming 1964 \\
\hline 185. & D. mincki (Karsch, 1890) & $25(24 \mathrm{~A}+\mathrm{X})$ & + & Brazil & Souza Bueno 1982 \\
\hline 186. & D. venosa (Burmeister, 1839) & $25(24 \mathrm{~A}+\mathrm{X})$ & + & Brazil & Kiauta and Boyes 1972 \\
\hline 187. & Diastatops intensa Montgomery, 1940 & $25(24 \mathrm{~A}+\mathrm{X})$ & + & Bolivia & Cumming 1964 \\
\hline 188. & D. obscura (Fabricius, 1775) & $25(24 \mathrm{~A}+\mathrm{X})$ & + & Bolivia & Cumming 1964 \\
\hline 189. & D. pullata (Burmeister, 1839) & $23(22 \mathrm{~A}+\mathrm{X})$ & + & Surinam & Kiauta 1979 a \\
\hline \multirow[t]{2}{*}{190.} & \multirow[t]{2}{*}{ Diplacodes bipunctata (Brauer, 1865) } & $25(24 \mathrm{~A}+\mathrm{X})$ & + & \multirow[t]{2}{*}{ Australia } & \multirow[t]{2}{*}{ Kiauta 1969b } \\
\hline & & $29(28 \mathrm{~A}+\mathrm{X})$ & + & & \\
\hline \multirow[t]{2}{*}{191.} & \multirow[t]{2}{*}{ D. haematodes (Burmeister, 1839) } & $25(24 \mathrm{~A}+\mathrm{X})$ & + & \multirow[t]{2}{*}{ Australia } & \multirow[t]{2}{*}{ Kiauta 1969b } \\
\hline & & $23(22 \mathrm{~A}+\mathrm{X})$ & - & & \\
\hline 192. & D. lefebvrei (Rambur, 1842) & $25(24 \mathrm{~A}+\mathrm{X})$ & + & Madagascar & Kiauta $1968 \mathrm{c}, 1969 \mathrm{~b}$ \\
\hline \multirow[t]{3}{*}{193.} & D. nebulosa (Fabricius, 1793) & $25(24 \mathrm{~A}+\mathrm{X})$ & + & India & Dasgupta 1957 \\
\hline & & $-»-$ & + & India & Kiauta and Kiauta 1982 \\
\hline & & $-n-$ & + & India & Tyagi 1982 \\
\hline 194. & D. trivialis (Rambur, 1842) & $25(24 \mathrm{~A}+\mathrm{X})$ & + & India & Asana and Makino 1935 \\
\hline & & $-»-$ & + & India & Makino 1935 \\
\hline & & $-n-$ & + & India & Dasgupta 1957 \\
\hline & & $-»-$ & + & Australia & Kiauta $1969 \mathrm{c}$ \\
\hline & & $-"-$ & + & Nepal & Kiauta 1975 \\
\hline & & $-»-$ & + & India & Tyagi 1982 \\
\hline 195. & Dythemis fugax Hagen, 1861 & $25(24 \mathrm{~A}+\mathrm{X})$ & + & USA & Cruden 1968 \\
\hline 196. & D. multipunctata Kirby, 1894 & $25(24 \mathrm{~A}+\mathrm{X})$ & + & Surinam & Kiauta 1979a \\
\hline & & $-»-$ & + & Brazil & Ferreira et al. 1979 \\
\hline 197. & D. rufinefris (Burmeister, 1839) & $25(24 \mathrm{~A}+\mathrm{X})$ & + & Jamaica & Cumming 1964 \\
\hline 198. & D. velox Hagen, 1861 & $25(24 \mathrm{~A}+\mathrm{X})$ & + & Bolivia & Cumming 1964 \\
\hline & & $-»-$ & + & Peru & Kiauta and Boyes 1972 \\
\hline 199. & $\begin{array}{l}\text { Elasmothemis cannacrioides } \\
\text { (Calvert, 1906) }\end{array}$ & $21(20 \mathrm{~A}+\mathrm{X})$ & - & Bolivia & $\begin{array}{l}\text { Cumming } 1964 \text { as Dythemis } \\
\text { cannacrioides Calvert, } 1906\end{array}$ \\
\hline & & $23(22 \mathrm{~A}+\mathrm{X})$ & + & Surinam & Kiauta 1979 a as D. cannacrioides \\
\hline & & $-»-$ & + & Brazil & Ferreira et al. 1979 \\
\hline 200. & E. williamsoni (Ris, 1919) & $22(20 \mathrm{~A}+$ neo-XY $)$ & - & Surinam & Kiauta $1979 \mathrm{a}$ as \\
\hline & & $25(24 \mathrm{~A}+\mathrm{X})$ & - & & Dythemis williamsoni (Ris, 1919) \\
\hline
\end{tabular}




\begin{tabular}{|c|c|c|c|c|c|}
\hline \multicolumn{2}{|c|}{ Taxon } & \multirow{2}{*}{$\begin{array}{c}\begin{array}{c}\text { Karyotype } \\
\text { formula } \mathbf{2 n} \delta\end{array} \\
25(24 \mathrm{~A}+\mathrm{X})\end{array}$} & \multirow{2}{*}{$\begin{array}{c}\begin{array}{c}\text { m-chromo } \\
\text { somes }\end{array} \\
- \\
\end{array}$} & \multirow{2}{*}{$\begin{array}{l}\text { Country } \\
\text { Bolivia } \\
\end{array}$} & \multirow{2}{*}{$\begin{array}{c}\text { References } \\
\text { Cumming } 1964 \\
\end{array}$} \\
\hline 201. & Erythemis attala (Selys, 1857) & & & & \\
\hline & & $-»-$ & + & Argentina & Agopian and Mola 1988 \\
\hline 202. & E. collocata (Hagen, 1861) & $25(24 \mathrm{~A}+\mathrm{X})$ & + & USA & Cruden 1968 \\
\hline 203. & E. credula (Hagen, 1861) & $25(24 A+X)$ & + & Surinam & Kiauta $1979 \mathrm{a}$ \\
\hline 204. & E. haematogastra (Burmeister, 1839) & $25(24 \mathrm{~A}+\mathrm{X})$ & - & Surinam & Kiauta $1979 \mathrm{a}$ \\
\hline 205. & E. peruviana (Rambur, 1842) & $25(24 A+X)$ & - & Surinam & Kiauta 1979a \\
\hline 206. & E. plebeja (Burmeister, 1839) & $25(24 \mathrm{~A}+\mathrm{X})$ & - & Bolivia & Cumming 1964 \\
\hline 207. & E. simplicicollis (Say, 1839) & $25(24 \mathrm{~A}+\mathrm{X})$ & + & USA & Cruden 1968 \\
\hline \multirow[t]{3}{*}{208.} & \multirow[t]{3}{*}{ E. vesiculosa (Fabricius, 1775) } & $25(24 \mathrm{~A}+\mathrm{X})$ & - & Bolivia & $\begin{array}{l}\text { Cumming } 1964 \text { as Lepthemis } \\
\text { vesiculosa (Fabricius, 1775) }\end{array}$ \\
\hline & & $-n-$ & - & Surinam & Kiauta 1979 a as L. vesiculosa \\
\hline & & $-n-$ & + & Brasil & Ferreira et al. 1979 as L. vesiculosa \\
\hline 209. & Erythrodiplax anomala (Brauer, 1865) & $25(24 A+X)$ & + & Brazil & Souza Bueno 1982 \\
\hline \multirow[t]{2}{*}{210.} & \multirow{2}{*}{ E. atroterminala Ris, 1911} & $25(24 \mathrm{~A}+\mathrm{X})$ & + & Uruguay & Goni and Abenante 1982 \\
\hline & & $-»-$ & + & Argentina & Mola 1996 \\
\hline \multirow[t]{2}{*}{211.} & \multirow[t]{2}{*}{ E. attenuata (Kirby, 1889) } & $25(24 \mathrm{~A}+\mathrm{X})$ & + & Surinam & Kiauta 1979a \\
\hline & & $-»-$ & + & Brasil & Ferreira et al. 1979 \\
\hline \multirow[t]{3}{*}{212.} & \multirow[t]{3}{*}{ E. basalis (Kirby, 1897) } & $25(24 \mathrm{~A}+\mathrm{X})$ & - & Bolivia & Cumming 1964 \\
\hline & & $-»-$ & + & Surinam & $\begin{array}{c}\text { Kiauta 1979a } \\
\text { (E. b. basalis (Kirby, 1897)) }\end{array}$ \\
\hline & & $-»-$ & + & Brasil & Ferreira et al. 1979 (E. b. basalis) \\
\hline \multirow[t]{3}{*}{213.} & \multirow[t]{3}{*}{ E. berenice (Drury, 1770) } & $25(24 \mathrm{~A}+\mathrm{X})$ & - & USA & Cruden 1968 \\
\hline & & $27(26 \mathrm{~A}+\mathrm{X})$ & + & \multirow[t]{2}{*}{ USA } & \multirow[t]{2}{*}{ Hung 1971} \\
\hline & & $25(24 \mathrm{~A}+\mathrm{X})$ & + & & \\
\hline 214. & E. castanea (Burmeister, 1839) & $25(24 \mathrm{~A}+\mathrm{X})$ & - & Bolivia & Cumming 1964 \\
\hline 215. & E. chromoptera Borror, 1942 & $23(22 \mathrm{~A}+\mathrm{X})$ & + & Uruguay & Goni and Abenante 1982 \\
\hline 216. & E. cleopatra Ris, 1911 & $25(24 \mathrm{~A}+\mathrm{X})$ & + & Peru & Kiauta and Boyes 1972 \\
\hline \multirow[t]{2}{*}{217.} & \multirow[t]{2}{*}{ E. connata (Burmeister, 1839) } & $25(24 \mathrm{~A}+\mathrm{X})$ & + & Chile & $\begin{array}{c}\text { Kiauta and Boyes } 1972 \\
\text { (E. c. connata (Burmeister, 1839)) }\end{array}$ \\
\hline & & $-»-$ & + & USA & $\begin{array}{c}\text { Kiauta and Brink } 1978 \\
\text { (E. c. minuscula (Rambur, 1842)) }\end{array}$ \\
\hline 218. & E. coralline (Brauer, 1865) & $25(24 \mathrm{~A}+\mathrm{X})$ & + & Argentina & Mola 1996 \\
\hline 219. & E. famula (Erichson, 1848) & $25(24 \mathrm{~A}+\mathrm{X})$ & + & Brazil & Souza Bueno 1982 \\
\hline 220. & E. fusca (Rambur, 1842) & $25(24 \mathrm{~A}+\mathrm{X})$ & - & Bolivia & $\begin{array}{l}\text { Cumming } 1964 \text { as E. connata fusca } \\
\text { (Rambur, 1842) }\end{array}$ \\
\hline & & $-»-$ & - & Guatemala & Cruden 1968 as E. c. fusca \\
\hline & & $-»-$ & + & Surinam & Kiauta 1979 a as E. c. fusca \\
\hline & & $-»-$ & + & Brazil & Ferreira et al. 1979 as E. c. fusca \\
\hline & & $-»-$ & + & Brazil & Souza Bueno 1982 \\
\hline & & $-»-$ & + & Argentina & Mola 1996 \\
\hline 221. & E. fervida (Erichson, 1848) & $25(24 \mathrm{~A}+\mathrm{X})$ & + & Jamaica & Cumming 1964 \\
\hline 222. & E. justiniana (Selys, 1857) & $25(24 \mathrm{~A}+\mathrm{X})$ & + & Jamaica & Cumming 1964 \\
\hline 223. & E. juliana Ris, 1911 & $25(24 \mathrm{~A}+\mathrm{X})$ & + & Brazil & Souza Bueno 1982 \\
\hline 224. & E. latimaculata Ris, 1911 & $25(24 \mathrm{~A}+\mathrm{X})$ & + & Surinam & Kiauta 1979a \\
\hline & & $-n-$ & + & Brasil & Ferreira et al. 1979 \\
\hline 225. & E. lygaea $\mathrm{Ris}, 1911$ & $25(24 \mathrm{~A}+\mathrm{X})$ & + & Argentina & Capitulo et al. 1991 \\
\hline & & $-»-$ & + & Argentina & Mola 1996 \\
\hline 226. & E. media Borror, 1942 & $21(20 \mathrm{~A}+\mathrm{X})$ & + & Bolivia & Cumming 1964 \\
\hline & & $22(20 \mathrm{~A}+\mathrm{XX})^{*}$ & + & Brazil & Kiauta and Boyes 1972 \\
\hline & & $21(20 \mathrm{~A}+\mathrm{X})$ & + & Surinam & Kiauta 1979a \\
\hline & & $-»-$ & + & Brasil & Ferreira et al. 1979 \\
\hline & & $22(20 \mathrm{~A}+$ neo-XY $)$ & + & Argentina & Mola 1996 \\
\hline 227. & E. melanorubra Borror, 1942 & $25(24 \mathrm{~A}+\mathrm{X})$ & + & Bolivia & Cumming 1964 \\
\hline & & $-»-$ & + & Venezuela & Kiauta and Boyes 1972 \\
\hline & & $-»-$ & + & Argentina & Capitulo et al. 1991 \\
\hline & & $-»-$ & + & Argentina & Mola 1996 \\
\hline 228. & E. minuscula (Rambur, 1842) & $25(24 \mathrm{~A}+\mathrm{X})$ & + & USA & Kiauta and Brink 1978 \\
\hline & & $22(20 A+n e o-X Y)$ & + & Argentina & Mola and Agopian 1985 \\
\hline 229. & E. nigricans (Rambur, 1842) & $25(24 \mathrm{~A}+\mathrm{X})$ & + & Uruguay & Goni and Abenante 1982 \\
\hline
\end{tabular}




\begin{tabular}{|c|c|c|c|c|c|}
\hline \multicolumn{2}{|c|}{ Taxon } & \multirow{2}{*}{$\begin{array}{c}\text { Karyotype } \\
\text { formula } 2 \mathbf{n} \delta \\
-»-\end{array}$} & \multirow{2}{*}{$\begin{array}{c}\begin{array}{c}\text { m-chromo } \\
\text { somes }\end{array} \\
+\end{array}$} & \multirow{2}{*}{$\begin{array}{l}\text { Country } \\
\text { Argentina } \\
\end{array}$} & \multirow{2}{*}{$\begin{array}{l}\text { References } \\
\text { Mola } 1996\end{array}$} \\
\hline 229. & E. nigricans (Rambur, 1842) & & & & \\
\hline & & $-»-$ & - & Argentina & De Gennaro 2004 \\
\hline & & $-n-$ & + & Argentina & De Gennaro et al. 2008 \\
\hline 230. & E. ochracea (Burmeister, 1839) & $25(24 A+X)$ & + & Argentina & Mola 1996 \\
\hline \multirow[t]{2}{*}{231.} & \multirow[t]{2}{*}{ E. paraguayensis (Foerster, 1904) } & $23(22 \mathrm{~A}+\mathrm{X})$ & + & Bolivia & Cumming 1964 \\
\hline & & $-n-$ & + & Surinam & Kiauta $1979 a$ \\
\hline \multirow[t]{5}{*}{232.} & \multirow[t]{5}{*}{ E. umbrata (Linnaeus, 1758) } & $25(24 \mathrm{~A}+\mathrm{X})$ & + & Bolivia & Cumming 1964 \\
\hline & & $-»-$ & + & Dominica & Cruden 1968 \\
\hline & & $-n-$ & + & Surinam & Kiauta 1979a \\
\hline & & $-n-$ & + & Brazil & Ferreira et al 1979 \\
\hline & & $-"-$ & + & Argentina & Mola 1996 \\
\hline \multirow[t]{2}{*}{233.} & \multirow[t]{2}{*}{ E. unimaculata (DeGeer, 1773) } & $25(24 \mathrm{~A}+\mathrm{X})$ & + & Bolivia & Cumming 1964 \\
\hline & & $-»-$ & + & Surinam & Kiauta 1979a \\
\hline 234. & Hydrobasileus croceus (Brauer, 1867) & $25(24 \mathrm{~A}+\mathrm{X})$ & + & India & Prasad and Thomas 1992 \\
\hline 235. & Ladona julia (Uhler, 1857) & $25(24 \mathrm{~A}+\mathrm{X})$ & + & USA & Cruden 1968 \\
\hline \multirow[t]{2}{*}{236.} & \multirow[t]{2}{*}{ Lathrecista asiatica (Fabricius, 1798) } & $25(24 \mathrm{~A}+\mathrm{X})$ & + & India & Dasgupta 1957 \\
\hline & & $-»-$ & + & India & Tyagi 1982 \\
\hline 237. & Leucorrhinia albifrons (Burmeister, 1839) & $25(24 \mathrm{~A}+\mathrm{X})$ & + & Former USSR & Makalowskaja 1940 \\
\hline \multirow[t]{2}{*}{238.} & \multirow{2}{*}{ L. dubia (Van der Linden, 1825) } & $26(24 \mathrm{~A}+\mathrm{XX})^{*}$ & - & Finland & Oksala 1939a, 1945 \\
\hline & & $25(24 \mathrm{~A}+\mathrm{X})$ & + & Russia & Kuznetsova et al. 2020b \\
\hline \multirow[t]{2}{*}{239.} & \multirow[t]{2}{*}{ L. frigida Hagen, 1890} & $21(20 A+X)$ & - & USA & Cruden 1968 \\
\hline & & $23(22 \mathrm{~A}+\mathrm{X})$ & + & & \\
\hline 240. & L. glacialis Hagen, 1890 & $25(24 \mathrm{~A}+\mathrm{X})$ & + & USA & Cruden 1968 \\
\hline 241. & L. hudsonica (Selys, 1850) & $25(24 \mathrm{~A}+\mathrm{X})$ & + & USA & Cruden 1968 \\
\hline & & $-»-$ & - & & \\
\hline 242. & L. intacta (Hagen, 1861) & $25(24 \mathrm{~A}+\mathrm{X})$ & + & USA & Cruden 1968 \\
\hline & & $-n-$ & - & & \\
\hline 243. & L. pectoralis (Charpentier, 1825) & $26(24 \mathrm{~A}+\mathrm{XX})^{*}$ & - & Finland & Oksala 1945 \\
\hline 244. & L. proxima Calvert, 1890 & $25(24 \mathrm{~A}+\mathrm{X})$ & + & USA & Cruden 1968 \\
\hline 245. & L. rubicunda (Linnaeus, 1857) & $25(24 \mathrm{~A}+\mathrm{X})$ & - & Finland & Oksala 1939a \\
\hline & & $-n-$ & - & Former USSR & Makalowskaja 1940 \\
\hline & & $-»-$ & - & Russia & Kuznetsova et al. 2018 \\
\hline 246. & Libellula angelina Selys, 1883 & $25(24 \mathrm{~A}+\mathrm{X})$ & + & Japan & Oguma 1915, 1930 \\
\hline & & $-n-$ & + & Japan & Kichijo 1942a \\
\hline 247. & L. auripennis Burmeister, 1839 & $25(24 \mathrm{~A}+\mathrm{X})$ & + & USA & Kiauta and Brink 1978 \\
\hline 248. & L. axilena Westwood, 1837 & $23(22 \mathrm{~A}+\mathrm{X})$ & - & USA & Cumming 1964 \\
\hline 249. & L. basalis (Say, 1840) & $25(24 \mathrm{~A}+\mathrm{X})$ & - & USA & Smith 1916 \\
\hline 250. & L. composita (Hagen, 1873) & $25(24 \mathrm{~A}+\mathrm{X})$ & + & USA & Cruden 1968 \\
\hline 251. & L. croceipennis Selys, 1868 & $25(24 \mathrm{~A}+\mathrm{X})$ & + & USA & Cruden 1968 \\
\hline 252. & L. cyanea Fabricius, 1775 & $25(24 \mathrm{~A}+\mathrm{X})$ & - & USA & Cruden 1968 \\
\hline 253. & L. depress $a$ Linnaeus, 1758 & $23(22 \mathrm{~A}+\mathrm{X})$ & - & Belgium & Carnoy 1885 \\
\hline & & $-»-$ & - & England & Hogben 1921 \\
\hline & & $25(24 \mathrm{~A}+\mathrm{X})$ & + & Austria & Kiauta 1968c, 1969b \\
\hline & & $23(22 \mathrm{~A}+\mathrm{X})$ & - & & \\
\hline & & $25(24 \mathrm{~A}+\mathrm{X})$ & + & France & Kiauta 1973b \\
\hline & & $-n-$ & + & Croatia & Francovič and Jurečic 1986, 1989 \\
\hline & & $-n-$ & + & Russia & Perepelov et al. 1998 \\
\hline & & $-»-$ & + & Russia & Kuznetsova et al. 2018 \\
\hline 254. & L. flavida Rambur, 1842 & $25(24 \mathrm{~A}+\mathrm{X})$ & + & USA & Cruden 1968 \\
\hline 255. & L. forensis Hagen, 1861 & $25(24 \mathrm{~A}+\mathrm{X})$ & + & USA & Cruden 1968 \\
\hline 256. & L. fulva Muller, 1764 & $25(24 \mathrm{~A}+\mathrm{X})$ & + & Switzerland & Kiauta and Kiauta 1979 \\
\hline & & $27(26 \mathrm{~A}+\mathrm{X})$ & + & Croatia & Francovič and Jurečic 1986, 1989 \\
\hline 257. & L. insecta Hagen, 1861 & $25(24 \mathrm{~A}+\mathrm{X})$ & - & USA & Cumming 1964 \\
\hline & & $-»-$ & - & USA & Cruden 1968 \\
\hline 258. & L. luctuosa Burmeister, 1839 & $25(24 \mathrm{~A}+\mathrm{X})$ & - & USA & Smith 1916 \\
\hline 259. & L. pulchella Drury, 1773 & $25(24 \mathrm{~A}+\mathrm{X})$ & + & USA & Cruden 1968 \\
\hline & & $-»-$ & + & Canada & Kiauta 1969a \\
\hline 260. & L. quadrimaculata Linnaeus, 1758 & $25(24 \mathrm{~A}+\mathrm{X})$ & + & Japan & $\begin{array}{c}\text { Oguma } 1915,1930 \\
\text { (L. q. asabinai Schmidt, 1957) }\end{array}$ \\
\hline
\end{tabular}




\begin{tabular}{|c|c|c|c|c|c|}
\hline \multicolumn{2}{|c|}{ Taxon } & \multirow{2}{*}{$\begin{array}{c}\begin{array}{c}\text { Karyotype } \\
\text { formula } \mathbf{2 n} \delta\end{array} \\
25(24 \mathrm{~A}+\mathrm{X}) \\
\end{array}$} & \multirow{2}{*}{$\begin{array}{c}\begin{array}{c}\text { m-chromo } \\
\text { somes }\end{array} \\
+ \\
\end{array}$} & \multirow{2}{*}{$\begin{array}{c}\text { Country } \\
\text { Japan } \\
\end{array}$} & \multirow{2}{*}{$\begin{array}{c}\text { References } \\
\text { Kichijo 1942d (L.q.asabinai) } \\
\end{array}$} \\
\hline \multirow[t]{10}{*}{260.} & \multirow[t]{10}{*}{ L. quadrimaculata Linnaeus, 1758} & & & & \\
\hline & & $-»-$ & + & Japan & Omura 1955 (L.q.asahinai) \\
\hline & & $-n-$ & + & Japan & Kiauta 1968b, c (L.q.asahinai) \\
\hline & & $-n-$ & + & Former USSR & $\begin{array}{c}\text { Fuchsówna and Sawczyńska } 1928 \text { (L. } \\
\text { q. quadrimaculata Linnaeus, 1758) }\end{array}$ \\
\hline & & $-"-$ & + & Finland & $\begin{array}{l}\text { Oksala 1939a, b, } 1945 \\
\text { (L.q. quadrimaculata) }\end{array}$ \\
\hline & & $-n-$ & + & Former USSR & $\begin{array}{l}\text { Makalowskaja } 1940 \\
\text { (L.q. quadrimaculata) }\end{array}$ \\
\hline & & $-"-$ & + & Netherlands & $\begin{array}{c}\text { Kiauta } 1968 \mathrm{~b}, \mathrm{c} \\
\text { (L. q. quadrimaculata) }\end{array}$ \\
\hline & & $-n-$ & + & USA & Cruden 1968 (L.q. quadrimaculata) \\
\hline & & $-n-$ & + & Russia & $\begin{array}{l}\text { Perepelov et al. } 1998 \\
\text { (L. q. quadrimaculata) }\end{array}$ \\
\hline & & $-"-$ & + & Russia & $\begin{array}{l}\text { Kuznetsova et al. } 2018 \\
\text { (L.q. quadrimaculata) }\end{array}$ \\
\hline 261. & L. saturata Uhler, 1857 & $25(24 A+X)$ & + & USA & Cruden 1968 \\
\hline 262. & L. semifasciata Burmeister, 1839 & $25(24 \mathrm{~A}+\mathrm{X})$ & + & USA & Cruden 1968 \\
\hline 263. & L. vibrans Fabricius, 1793 & $25(24 A+X)$ & + & USA & Cruden 1968 \\
\hline 264. & Lyriothemis pachygastra (Selys, 1878) & $25(24 \mathrm{~A}+\mathrm{X})$ & - & Japan & Omura 1955 \\
\hline 265. & Macrothemis declivata Calvert, 1909 & $23(22 \mathrm{~A}+\mathrm{X})$ & + & Brazil & Kiauta and Boyes 1972 \\
\hline 266. & M. hemichlora (Burmeister, 1839) & $6(4 \mathrm{~A}+$ neo-XY $)$ & - & Bolivia & Cumming 1964 \\
\hline 267. & M. imitans Karsch, 1890 & $25(24 \mathrm{~A}+\mathrm{X})$ & + & Brazil & $\begin{array}{l}\text { Kiauta and Boyes } 1972 \\
\text { (M. i. imitans Karsch, 1890) }\end{array}$ \\
\hline 268. & M. mortoni Ris, 1913 & $25(24 \mathrm{~A}+\mathrm{X})$ & + & Bolivia & Cumming 1964 \\
\hline 269. & M. musiva Calvert, 1898 & $25(24 \mathrm{~A}+\mathrm{X})$ & + & Bolivia & Cumming 1964 \\
\hline 270. & Macrothemis sp. & $25(24 \mathrm{~A}+\mathrm{X})$ & + & Argentina & Mola 2007 \\
\hline 271. & Miathyria artemis (Selys, 1857) & $25(24 \mathrm{~A}+\mathrm{X})$ & + & Surinam & Kiauta 1979a \\
\hline \multirow[t]{4}{*}{272.} & \multirow[t]{4}{*}{ M. marcella (Selys, 1857) } & $25(24 \mathrm{~A}+\mathrm{X})$ & + & Bolivia & Cumming 1964 \\
\hline & & $-n-$ & + & Surinam & Kiauta $1979 \mathrm{a}$ \\
\hline & & $-n-$ & + & Argentina & Mola and Agopian 1985 \\
\hline & & $-»-$ & + & Brazil & Ferreira et al. 1979 \\
\hline \multirow[t]{2}{*}{273.} & \multirow[t]{2}{*}{ Micrathyria artemis Ris, 1911} & $25(24 \mathrm{~A}+\mathrm{X})$ & + & Brazil & Ferreira et al. 1979 \\
\hline & & $-n-$ & + & Brazil & Souza Bueno 1982 \\
\hline 274. & M. atra (Martin, 1897) & $25(24 \mathrm{~A}+\mathrm{X})$ & + & Bolivia & Cumming 1964 \\
\hline \multirow[t]{2}{*}{275.} & \multirow[t]{2}{*}{ M. catenata Calvert, 1909} & $25(24 \mathrm{~A}+\mathrm{X})$ & + & Brazil & Souza Bueno 1982 \\
\hline & & $-n-$ & + & Argentina & Mola 2007 \\
\hline 276. & M. didyma (Selys, 1857) & $25(24 A+X)$ & + & Jamaica & Cumming 1964 \\
\hline 277. & M. exima Kirby, 1897 & $25(24 \mathrm{~A}+\mathrm{X})$ & + & Surinam & Kiauta 1979a \\
\hline 278. & M. hagenii Kirby, 1890 & $25(24 A+X)$ & + & Jamaica & Cumming 1964 \\
\hline \multirow[t]{3}{*}{279.} & \multirow[t]{3}{*}{ M. hesperis Ris, 1911} & $25(24 \mathrm{~A}+\mathrm{X})$ & + & Surinam & Kiauta 1979 a \\
\hline & & $-n-$ & + & Brazil & Ferreira et al. 1979 \\
\hline & & $-n-$ & + & Argentina & Mola et al. 1999 \\
\hline \multirow[t]{2}{*}{280.} & \multirow[t]{2}{*}{ M. hypodydima Calvert 1906} & $23(22 \mathrm{~A}+\mathrm{X})$ & + & Brazil & Souza Bueno 1982 \\
\hline & & $25(24 \mathrm{~A}+\mathrm{X})$ & + & Argentina & Agopian and Mola 1988 \\
\hline 281. & M. iheringi Santos, 1946 & $23(22 \mathrm{~A}+\mathrm{X})$ & + & Bolivia & Cumming 1964 \\
\hline \multirow[t]{2}{*}{282.} & \multirow[t]{2}{*}{ M. laevigata Calvert, 1909} & $25(24 \mathrm{~A}+\mathrm{X})$ & + & Bolivia & Cumming 1964 \\
\hline & & $-»-$ & + & Brazil & Kiauta and Boyes 1972 \\
\hline 283. & M. longifasciata Calvert, 1909 & $24(22 \mathrm{~A}+$ neo-XY $)$ & - & Argentina & Agopian and Mola 1988 \\
\hline 284. & M. ocellata (Martin, 1897) & $25(24 \mathrm{~A}+\mathrm{X})$ & + & Bolivia & $\begin{array}{c}\text { Cumming } 1964 \\
\text { (M. o. dentiens Calvert, 1909) } \\
\end{array}$ \\
\hline \multirow[t]{2}{*}{285.} & M. spuria (Selys, 1900) & $25(24 \mathrm{~A}+\mathrm{X})$ & + & Bolivia & Cumming 1964 \\
\hline & & $-n-$ & + & Argentina & Mola et al. 1999 \\
\hline 286. & M. stawiarskii Santos, 1953 & $25(24 \mathrm{~A}+\mathrm{X})$ & + & Brazil & Souza Bueno 1982 \\
\hline 287. & M. ungulata Foerster, 1907 & $23\left(20 \mathrm{~A}+\mathrm{X}_{1} \mathrm{X}_{2} \mathrm{Y}\right)$ & - & Argentina & Mola et al. 1999 \\
\hline 288. & M. cf. eximia Kirby, 1879 & $21(20 \mathrm{~A}+\mathrm{X})$ & - & Bolivia & Cumming 1964 \\
\hline 289. & M. sp. (ungulata Foerster, 1907-group) & $23(22 \mathrm{~A}+\mathrm{X})$ & - & Bolivia & Cumming 1964 \\
\hline 290. & Nannothemis bella (Uhler, 1857) & $25(24 A+X)$ & + & USA & Cruden 1968 \\
\hline 291. & Nesciothemis farinosa (Foerster, 1898) & $25(24 \mathrm{~A}+\mathrm{X})$ & + & Kenya & Kiauta 1969c \\
\hline & & $-n-$ & + & Kenya & Wasscher 1985 \\
\hline
\end{tabular}




\begin{tabular}{|c|c|c|c|c|c|}
\hline \multicolumn{2}{|c|}{ Taxon } & \multirow{2}{*}{$\begin{array}{c}\begin{array}{c}\text { Karyotype } \\
\text { formula } \mathbf{2 n} \delta\end{array} \\
25(24 \mathrm{~A}+\mathrm{X})\end{array}$} & \multirow{2}{*}{\begin{tabular}{c|}
$\begin{array}{c}\text { m-chromo } \\
\text { somes }\end{array}$ \\
+
\end{tabular}} & \multirow{2}{*}{$\begin{array}{l}\text { Country } \\
\text { Hawaii }\end{array}$} & \multirow{2}{*}{$\begin{array}{c}\text { References } \\
\text { Kiauta 1969d }\end{array}$} \\
\hline 292. & Nesogonia blackburni (McLachlan, 1883) & & & & \\
\hline 293. & Neurothemis fulvia (Drury, 1773) & $25(24 \mathrm{~A}+\mathrm{X})$ & + & Nepal & Kiauta 1974,1975 \\
\hline \multirow[t]{2}{*}{294.} & \multirow[t]{2}{*}{ N. intermedia (Rambur, 1842) } & $25(24 \mathrm{~A}+\mathrm{X})$ & + & Nepal & $\begin{array}{c}\text { Kiauta 1974, } 1975 \text { (N. i. intermedia } \\
\text { (Rambur, 1842)) }\end{array}$ \\
\hline & & $-n-$ & + & Nepal & $\begin{array}{l}\text { Kiauta and Kiauta } 1982 \text { (N. i. degener } \\
\text { (Sel, 1842)) }\end{array}$ \\
\hline 295. & N. terminata Ris, 1911 & $25(24 \mathrm{~A}+\mathrm{X})$ & + & Philippines & Kiauta and Kiauta 1980b \\
\hline \multirow[t]{4}{*}{296.} & \multirow{4}{*}{ N. tullia (Drury, 1773) } & $28(26 \mathrm{~A}+$ neo-XY $)$ & + & India & Ray Chaudhuri and Dasgupta 1949 \\
\hline & & $-»-$ & + & India & $\begin{array}{c}\text { Kiauta 1969a (N. t. tullia } \\
\text { (Drury, 1773)) }\end{array}$ \\
\hline & & $-n-$ & + & India & Tyagi 1982 (N.t.tullia) \\
\hline & & $25(24 \mathrm{~A}+\mathrm{X})$ & + & Thailand & Kiauta and Kiauta 1983 \\
\hline 297. & Oligoclada amphinome Ris, 1919 & $25(24 \mathrm{~A}+\mathrm{X})$ & + & Surinam & Kiauta 1979a \\
\hline \multirow[t]{2}{*}{298.} & \multirow[t]{2}{*}{ O. Laetitia Ris, 1911} & $23(22 \mathrm{~A}+\mathrm{X})$ & + & Argentina & Mola and Agopian 1985 \\
\hline & & $21(20 \mathrm{~A}+\mathrm{X})$ & - & Brazil & Souza Bueno 1982 \\
\hline \multirow[t]{2}{*}{299.} & \multirow[t]{2}{*}{ O. monosticha Borror, 1931} & $23(22 \mathrm{~A}+\mathrm{X})$ & + & Surinam & Kiauta 1979 a \\
\hline & & $-n-$ & + & Brazil & Ferreira et al. 1979 \\
\hline 300. & O. pachystigma Karsch, 1890 & $23(22 \mathrm{~A}+\mathrm{X})$ & + & Brazil & Souza Bueno 1982 \\
\hline 301. & Orthemis aequilibris Calvert, 1909 & $12(10 \mathrm{~A}+$ neo-XY $)$ & - & Surinam & Kiauta $1979 \mathrm{a}$ \\
\hline 302. & O. ambinigra Calvert, 1909 & $12(10 \mathrm{~A}+$ neo-XY) & - & Argentina & Agopian and Mola 1984 \\
\hline 303. & O. biolleyi Calvert, 1906 & $23(22 \mathrm{~A}+\mathrm{X})$ & + & Bolivia & Cumming 1964 \\
\hline \multirow[t]{3}{*}{304.} & \multirow[t]{3}{*}{ O. cultiformis Calvert, 1906} & $23(22 \mathrm{~A}+\mathrm{X})$ & + & Bolivia & Cumming 1964 \\
\hline & & $-»-$ & + & Surinam & Kiauta 1979a \\
\hline & & $-n-$ & + & Brazil & Ferreira et al. 1979 \\
\hline 305. & O. discolor Burmeister, 1839 & $23(22 \mathrm{~A}+\mathrm{X})$ & + & Argentina & Mola 2007 \\
\hline \multirow[t]{10}{*}{306.} & \multirow[t]{10}{*}{ O. ferruginea (Fabricius, 1775) } & $10(8 \mathrm{~A}+\mathrm{neo}-\mathrm{XY})^{* * *}$ & - & Bolivia & \multirow[t]{2}{*}{ Cumming 1964} \\
\hline & & $23(22 \mathrm{~A}+\mathrm{X})$ & - & USA & \\
\hline & & $-n-$ & + & $\begin{array}{l}\text { Guatemala, } \\
\text { Dominica }\end{array}$ & Cruden 1968 \\
\hline & & $-»-$ & + & Peru & Kiauta 1969a, 1971c \\
\hline & & $-n-$ & + & Peru & Kiauta and Boyes 1972 \\
\hline & & $23(22 \mathrm{~A}+\mathrm{X})$ & + & \multirow[t]{2}{*}{ Surinam } & \multirow[t]{2}{*}{ Kiauta 1979a } \\
\hline & & $25(24 \mathrm{~A}+\mathrm{X})$ & + & & \\
\hline & & $23(22 \mathrm{~A}+\mathrm{X})$ & + & Brazil & Ferreira et al. 1979 \\
\hline & & $23(22 \mathrm{~A}+\mathrm{X})$ & - & Brazil, Argentina & Mola and Agopian 1985 \\
\hline & & $24(22 \mathrm{~A}+\mathrm{XX})^{*}$ & + & & \\
\hline 307. & O. Levis Calvert, 1906 & $6(4 \mathrm{~A}+\text { neo-XY })^{* * *}$ & - & Bolivia & Cumming 1964 \\
\hline & & $8(6 \mathrm{~A}+\mathrm{neo}-\mathrm{XY})^{* * *}$ & - & & \\
\hline 308. & O. nodiplaga Karsch, 1891 & $41(40 \mathrm{~A}+\mathrm{X})$ & - & Argentina & Agopian and Mola 1984 \\
\hline 309. & Orthetrum abbotti Calvert, 1892 & $25(24 \mathrm{~A}+\mathrm{X})$ & + & $\begin{array}{c}\text { Kingdom of } \\
\text { Eswatini (Former } \\
\text { Swaziland) }\end{array}$ & Boyes et al. 1980 \\
\hline 310. & O. albistylum (Selys, 1848) & $25(24 \mathrm{~A}+\mathrm{X})$ & + & Italy & $\begin{array}{l}\text { Kiauta 1971a (O. a. albistylum } \\
\text { (Selys, 1848)) }\end{array}$ \\
\hline & & $-n-$ & + & Russia & Perepelov et al. 1998 \\
\hline & & $-»-$ & + & Japan & $\begin{array}{c}\text { Oguma 1915, 1917, 1930 } \\
\text { (O. a. speciosum (Uhler, 1858)) }\end{array}$ \\
\hline & & $-n-$ & + & India & Kichijo 1942b (O. a. speciosum) \\
\hline & & $-n-$ & + & Japan & Omura 1955 (O. a. speciosum) \\
\hline 311. & O. azureum (Rambur, 1842) & $25(24 \mathrm{~A}+\mathrm{X})$ & + & Madagascar & Kiauta 1969b, c \\
\hline 312. & O. brachiale (Beauvois, 1805) & $21(20 \mathrm{~A}+\mathrm{X})$ & - & Kenya & Kiauta 1969b, c \\
\hline & & $25(24 \mathrm{~A}+\mathrm{X})$ & + & $\begin{array}{c}\text { Burkina Faso } \\
\text { (Former Voltiac } \\
\text { Republic) }\end{array}$ & $\begin{array}{c}\text { Kiauta and Ochssée } 1979 \\
\text { (O. b. brachiale (Beauvois, 1805)) }\end{array}$ \\
\hline 313. & O. brunneum (Fonscolombe, 1837) & $25(24 \mathrm{~A}+\mathrm{X})$ & + & Italy & Kiauta 1971a \\
\hline & & $-»-$ & + & Russia & Perepelov et al. 1998 \\
\hline 314. & O. cancellatum (Linnaeus, 1758) & $25(24 \mathrm{~A}+\mathrm{X})$ & + & Finland & Oksala 1939a \\
\hline & & $-»-$ & + & India & Dasgupta 1957 \\
\hline & & $-n-$ & + & Netherlands & Kiauta 1969a, b \\
\hline & & $-n-$ & + & India & Tyagi 1982 \\
\hline & & $-n-$ & + & Russia & Kuznetsova et al. 2018 \\
\hline
\end{tabular}




\begin{tabular}{|c|c|c|c|c|c|}
\hline \multicolumn{2}{|c|}{ Taxon } & \multirow{2}{*}{$\begin{array}{c}\text { Karyotype } \\
\text { formula } \mathbf{2 n} \delta \\
25(24 \mathrm{~A}+\mathrm{X})\end{array}$} & \multirow{2}{*}{$\begin{array}{c}\begin{array}{c}\text { m-chromo } \\
\text { somes }\end{array} \\
+\end{array}$} & \multirow{2}{*}{$\begin{array}{c}\text { Country } \\
\text { Burkina Faso } \\
\text { (Former Voltiac } \\
\text { Republic) }\end{array}$} & \multirow{2}{*}{$\begin{array}{c}\text { References } \\
\text { Kiauta and Ochssée } 1979\end{array}$} \\
\hline 315. & O. chrysostigma (Burmeister, 1839) & & & & \\
\hline & & $-n-$ & + & $\begin{array}{l}\text { Kingdom of } \\
\text { Eswatini (Former } \\
\text { Swaziland) }\end{array}$ & Boyes et al. 1980 \\
\hline & & $-"-$ & + & Kenya & Wasscher 1985 \\
\hline \multirow[t]{4}{*}{316.} & \multirow[t]{4}{*}{ O. coerulescens (Fabricius, 1798) } & $25(24 \mathrm{~A}+\mathrm{X})$ & + & \multirow[t]{2}{*}{ Austria } & \multirow[t]{2}{*}{ Kiauta $1969 \mathrm{c}$} \\
\hline & & $23(22 \mathrm{~A}+\mathrm{X})$ & - & & \\
\hline & & $25(24 \mathrm{~A}+\mathrm{X})$ & + & \multirow[t]{2}{*}{ Italy } & \multirow[t]{2}{*}{ Kiauta 1971a } \\
\hline & & $27(26 \mathrm{~A}+\mathrm{X})$ & + & & \\
\hline \multirow[t]{7}{*}{317.} & \multirow[t]{7}{*}{ O. glaucum (Brauer, 1865) } & $25(24 \mathrm{~A}+\mathrm{X})$ & + & India & Dasgupta 1957 \\
\hline & & $-»-$ & + & India & Tyagi $1978 \mathrm{a}, \mathrm{b}$ \\
\hline & & $-"-$ & + & India & Handa and Batra 1980 \\
\hline & & $-»-$ & + & India & Tyagi 1982 \\
\hline & & $-»-$ & + & India & Handa et al. 1984 \\
\hline & & $-»-$ & + & India & Walia and Sandhu 2002 \\
\hline & & $-"-$ & + & India & Kumari and Gautam 2017 \\
\hline 318. & O. guineese (Ris, 1909) & $25(24 \mathrm{~A}+\mathrm{X})$ & + & $\begin{array}{l}\text { Burkina Faso } \\
\text { (Former Voltiac } \\
\text { Republic) }\end{array}$ & Kiauta and Ochssée 1979 \\
\hline \multirow[t]{5}{*}{319.} & \multirow[t]{5}{*}{ O. japonicum (Uhler, 1858) } & $25(24 \mathrm{~A}+\mathrm{X})$ & + & Japan & $\begin{array}{c}\text { Oguma } 1917,1930(O . j . \text { internum } \\
\text { McLachlan, 1894) }\end{array}$ \\
\hline & & $-n-$ & + & Japan & Kichijo 1942b (O.j. internum) \\
\hline & & $-»-$ & + & Japan & Omura 1955 (O. j. internum) \\
\hline & & $-n-$ & + & Nepal & Kiauta $1975(O . j$. internum $)$ \\
\hline & & $-"-$ & + & Nepal & $\begin{array}{c}\text { Kiauta and Kiauta } 1976 \\
(\text { O. j. internum })\end{array}$ \\
\hline \multirow[t]{2}{*}{320.} & \multirow[t]{2}{*}{ O. julia Kirby, 1900} & $25(24 \mathrm{~A}+\mathrm{X})$ & + & $\begin{array}{c}\text { Kingdom of } \\
\text { Eswatini (Former } \\
\text { Swaziland) }\end{array}$ & $\begin{array}{l}\text { Boyes et al. } 1980(O . j . \text { falsum } \\
\quad \text { (Longfeild, 1955)) }\end{array}$ \\
\hline & & $-»-$ & + & Kenya & Wasscher 1985 (O. j. falsum) \\
\hline \multirow[t]{4}{*}{321.} & \multirow[t]{4}{*}{ O. luzonicum (Brauer, 1868) } & $25(24 \mathrm{~A}+\mathrm{X})$ & + & Nepal & Kiauta 1975 \\
\hline & & $-n-$ & + & Nepal & Kiauta and Kiauta 1982 \\
\hline & & $-n-$ & + & India & Thomas and Prasad 1981 \\
\hline & & $-n-$ & + & India & Prasad and Thomas 1992 \\
\hline \multirow[t]{3}{*}{322.} & \multirow[t]{3}{*}{ O. melania (Selys, 1883) } & $25(24 \mathrm{~A}+\mathrm{X})$ & + & Japan & Oguma 1917 \\
\hline & & $-»-$ & + & Japan & Omura 1955 \\
\hline & & $-n-$ & + & Russia & Perepelov 2003 \\
\hline 323. & O. monardi (Schmidt, 1951) & $25(24 \mathrm{~A}+\mathrm{X})$ & + & $\begin{array}{c}\text { Burkina Faso } \\
\text { (Former Voltiac } \\
\text { Republic) } \\
\end{array}$ & Kiauta and Ochssée 1979 \\
\hline 324. & O. poecilops (Ris, 1916) & $25(24 \mathrm{~A}+\mathrm{X})$ & + & Japan & $\begin{array}{c}\text { Suzuki et al. } 1991(O . p . \\
\text { miyajimaensis Yuki et Doi, 1938) }\end{array}$ \\
\hline \multirow[t]{8}{*}{325.} & \multirow[t]{8}{*}{ O. pruinosum (Burmeister, 1839) } & $25(24 \mathrm{~A}+\mathrm{X})$ & + & India & $\begin{array}{c}\text { Dasgupta } 1957 \text { (O.p. neglectum } \\
\text { (Rambur, 1842)) }\end{array}$ \\
\hline & & $-"-$ & + & Taiwan & Kiauta 1969a, c (O.p. neglectum) \\
\hline & & $-"-$ & + & India & Tyagi $1982($ O. p. neglectum $)$ \\
\hline & & $-"-$ & + & India & $\begin{array}{c}\text { Prasad and Thomas } 1992 \\
(\text { O. p. neglectum })\end{array}$ \\
\hline & & $-n-$ & + & India & Tyagi 1978a, b (O. p. neglectum) \\
\hline & & $-"-$ & + & Nepal & $\begin{array}{c}\text { Kiauta and Kiauta } 1982 \\
(\text { O.p. neglectum })\end{array}$ \\
\hline & & $-"-$ & + & India & $\begin{array}{l}\text { Walia and Sandhu } 2002 \\
(\text { O.p. neglectum })\end{array}$ \\
\hline & & $-"-$ & + & India & $\begin{array}{l}\text { Kumari and Gautam } 2017 \\
\text { (O.p. neglectum })\end{array}$ \\
\hline \multirow[t]{5}{*}{326.} & O. sabina (Drury, 1773) & $25(24 \mathrm{~A}+\mathrm{X})$ & + & India & Asana and Makino 1935 \\
\hline & & $-»-$ & + & India & Makino 1935 \\
\hline & & $-n-$ & + & India & Kichijo 1942b \\
\hline & & $-n-$ & + & India & Ray Chaudhuri and Dasgupta 1949 \\
\hline & & $-n-$ & + & Nepal & Kiauta 1975 \\
\hline
\end{tabular}




\begin{tabular}{|c|c|c|c|c|c|}
\hline \multicolumn{2}{|c|}{ Taxon } & \multirow{2}{*}{$\begin{array}{c}\begin{array}{c}\text { Karyotype } \\
\text { formula } 2 \mathbf{n}\end{array} \\
-»-\end{array}$} & \multirow{2}{*}{$\begin{array}{c}\begin{array}{c}\text { m-chromo } \\
\text { somes }\end{array} \\
+\end{array}$} & \multirow{2}{*}{$\begin{array}{c}\text { Country } \\
\text { India }\end{array}$} & \multirow{2}{*}{$\begin{array}{l}\text { References } \\
\text { Tyagi } 1982 \\
\end{array}$} \\
\hline 326. & O. sabina (Drury, 1773) & & & & \\
\hline & & $-n-$ & + & India & Prasad and Thomas 1992 \\
\hline & & $-»-$ & + & India & $\begin{array}{l}\text { Walia and Sandhu } 2002 \\
\text { (O. s. sabina (Drury, 1773)) }\end{array}$ \\
\hline \multirow[t]{9}{*}{327.} & \multirow[t]{9}{*}{ O. taeniolatum (Schneider, 1845) } & $25(24 \mathrm{~A}+\mathrm{X})$ & + & Greece & Kiauta $1972 \mathrm{a}$ \\
\hline & & $-»-$ & + & Nepal & Kiauta 1975 \\
\hline & & $-"-$ & + & India & Tyagi 1978a, b \\
\hline & & $-n-$ & + & India & Handa and Batra 1980 \\
\hline & & $-n-$ & + & India & Tyagi 1982 \\
\hline & & $-n-$ & + & India & Handa et al. 1984 \\
\hline & & $-n-$ & + & India & Thomas and Prasad 1986 \\
\hline & & $-n-$ & + & India & Walia and Sandhu 2002a \\
\hline & & $-»-$ & + & India & Walia et al. 2015 \\
\hline 328. & O. testaceum (Burmeister, 1839) & $25(24 \mathrm{~A}+\mathrm{X})$ & + & Nepal & Kiauta and Kiauta 1982 \\
\hline \multirow[t]{7}{*}{329.} & \multirow[t]{7}{*}{ O. triangulare (Selys, 1878) } & $25(24 \mathrm{~A}+\mathrm{X})$ & + & Japan & $\begin{array}{c}\text { Omura } 1955 \\
\text { (O. t. melania }(\text { Selys, 1883)) }\end{array}$ \\
\hline & & $-n-$ & + & Taiwan & $\begin{array}{c}\text { Kiauta 1969a, b (O. t. triangulare } \\
\text { (Selys, 1878)) }\end{array}$ \\
\hline & & $-n-$ & + & Nepal & Kiauta 1975 (O.t. triangulare) \\
\hline & & $-"-$ & + & India & Tyagi 1978a, b (O. t. triangulare) \\
\hline & & $-»-$ & + & India & $\begin{array}{c}\text { Handa and Batra } 1980 \\
\text { (O.t.triangulare })\end{array}$ \\
\hline & & $-n-$ & + & India & Tyagi 1982 (O. t. triangulare) \\
\hline & & $-"-$ & + & India & $\begin{array}{c}\text { Walia and Sandhu } 2002 \text { (O.t. } \\
\text { triangulare) }\end{array}$ \\
\hline \multirow[t]{3}{*}{330.} & \multirow{3}{*}{$\begin{array}{l}\text { Pachydiplax longipennis (Burmeister, } \\
\text { 1839) }\end{array}$} & $25(24 \mathrm{~A}+\mathrm{X})$ & - & USA & Cumming 1964 \\
\hline & & $-»-$ & + & USA & Cruden 1968 \\
\hline & & $-n-$ & + & USA & Kiauta and Brink 1978 \\
\hline 331. & Palpopleura jucunda Rambur, 1842 & $25(24 \mathrm{~A}+\mathrm{X})$ & + & $\begin{array}{c}\text { Kingdom of } \\
\text { Eswatini (Former } \\
\text { Swaziland) } \\
\end{array}$ & Boyes et al. 1980 \\
\hline \multirow[t]{2}{*}{332.} & \multirow[t]{2}{*}{ P. lucia (Drury, 1773) } & $25(24 \mathrm{~A}+\mathrm{X})$ & + & $\begin{array}{c}\text { Burkina Faso } \\
\text { (Former Voltiac } \\
\text { Republic) }\end{array}$ & $\begin{array}{c}\text { Kiauta and Ochssée } 1979 \text { (P. l. portia } \\
\text { (Drury, 1773)) }\end{array}$ \\
\hline & & $-»-$ & + & Kenya & Wasscher 1985 (P. l. portia) \\
\hline \multirow[t]{2}{*}{333.} & \multirow[t]{2}{*}{ P. sexmaculata (Fabricius, 1787) } & $25(24 \mathrm{~A}+\mathrm{X})$ & + & Nepal & Kiauta 1974,1975 \\
\hline & & $-»-$ & + & India & $\begin{array}{l}\text { Tyagi } 1982 \text { (P. s. sexmaculata } \\
\text { (Fabricius, 1787)) }\end{array}$ \\
\hline \multirow[t]{15}{*}{334.} & \multirow[t]{15}{*}{ Pantala flavescens (Fabricius, 1798) } & $25(24 \mathrm{~A}+\mathrm{X})$ & + & India & Asana and Makino 1935 \\
\hline & & $-»-$ & + & India & Makino 1935 \\
\hline & & $-n-$ & + & India & Kichijo 1942b \\
\hline & & $-»-$ & + & India & Dasgupta 1957 \\
\hline & & $-»-$ & + & India & Seshachar and Bagga 1963 \\
\hline & & $-»-$ & + & Bolivia & Cumming 1964 \\
\hline & & $-»-$ & + & Madagascar & Kiauta 1969b \\
\hline & & $-n-$ & + & Surinam & Kiauta 1979a \\
\hline & & $-»-$ & + & Brazil & Ferreira et al. 1979 \\
\hline & & $-»-$ & + & $\begin{array}{c}\text { Kingdom of } \\
\text { Eswatini (Former } \\
\text { Swaziland) } \\
\end{array}$ & Boyes et al. 1980 \\
\hline & & $-n-$ & + & Brazil & Souza Bueno 1982 \\
\hline & & $-n-$ & + & Argentina & Agopian and Mola 1988 \\
\hline & & $-n-$ & + & India & Prasad and Thomas 1992 \\
\hline & & $-»-$ & + & Russia & Perepelov and Bugrov 2001b \\
\hline & & $23(22 \mathrm{~A}+\mathrm{X})$ & + & India & Walia et al. 2011 \\
\hline \multirow[t]{2}{*}{335.} & \multirow[t]{2}{*}{ P. hymenaea (Say, 1836) } & $25(24 \mathrm{~A}+\mathrm{X})$ & + & Bolivia & Cumming 1964 \\
\hline & & $-»-$ & + & USA & Cruden 1968 \\
\hline 336. & Perithemis cornelia Ris, 1910 & $25(24 \mathrm{~A}+\mathrm{X})$ & - & Bolivia & Cumming 1964 \\
\hline 337. & P. domitia (Drury, 1773) & $25(24 \mathrm{~A}+\mathrm{X})$ & + & Jamaica & Cumming 1964 \\
\hline 338. & P. electra Ris, 1928 & $25(24 \mathrm{~A}+\mathrm{X})$ & - & Bolivia & Cumming 1964 \\
\hline 339. & P. icteroptera (Selys in Sagra, 1857) & $25(24 \mathrm{~A}+\mathrm{X})$ & + & Argentina & Mola and Agopian 1985 \\
\hline
\end{tabular}




\begin{tabular}{|c|c|c|c|c|c|}
\hline \multicolumn{2}{|c|}{ Taxon } & \multirow{2}{*}{$\begin{array}{c}\begin{array}{c}\text { Karyotype } \\
\text { formula } \mathbf{2 n} \delta\end{array} \\
17(16 \mathrm{~A}+\mathrm{X})\end{array}$} & \multirow{2}{*}{$\begin{array}{c}\begin{array}{c}\text { m-chromo } \\
\text { somes }\end{array} \\
-\end{array}$} & \multirow{2}{*}{$\begin{array}{l}\text { Country } \\
\text { Bolivia }\end{array}$} & \multirow{2}{*}{$\begin{array}{c}\text { References } \\
\text { Cumming } 1964\end{array}$} \\
\hline 340. & P. lais (Petry, 1834) & & & & \\
\hline & & $-»-$ & - & Surinam & Kiauta 1979 a \\
\hline & & $-"-$ & - & Brazil & Ferreira et al. 1979 \\
\hline \multirow[t]{4}{*}{341.} & \multirow[t]{4}{*}{ P. mooma Kirby, 1889} & $25(24 \mathrm{~A}+\mathrm{X})$ & + & Bolivia & Cumming 1964 \\
\hline & & $-»-$ & + & Surinam & Kiauta 1979a \\
\hline & & $-n-$ & - & Brazil & Ferreira et al. 1979 \\
\hline & & $-»-$ & + & Argentina & Mola and Agopian 1985 \\
\hline 342. & P. tenera (Say, 1839) & $25(24 \mathrm{~A}+\mathrm{X})$ & + & USA & Kiauta and Brink 1978 \\
\hline 343. & P. seminole Calvert, 1907 & $25(24 \mathrm{~A}+\mathrm{X})$ & + & USA & Cumming 1964 \\
\hline 344. & Perithemis sp. & $25(24 \mathrm{~A}+\mathrm{X})$ & - & Bolivia & Cumming 1964 \\
\hline \multirow[t]{2}{*}{345.} & \multirow[t]{2}{*}{ Planiplax erythropyga (Karsch, 1891) } & $25(24 \mathrm{~A}+\mathrm{X})$ & + & Argentina & Mola et al. 1999 \\
\hline & & $-n-$ & + & $-»-$ & De Gennaro 2004 \\
\hline 346. & P. sanguiniventris (Calvert, 1907) & $25(24 \mathrm{~A}+\mathrm{X})$ & + & USA & Cruden 1968 \\
\hline \multirow[t]{2}{*}{347.} & \multirow[t]{2}{*}{ Plathemis lydia (Drury, 1773) } & $25(24 \mathrm{~A}+\mathrm{X})$ & + & USA & McGill 1907 \\
\hline & & $-»-$ & + & USA & Cruden 1968 \\
\hline \multirow[t]{7}{*}{348.} & \multirow[t]{7}{*}{ Potamarcha congener (Rambur, 1842) } & $25(24 \mathrm{~A}+\mathrm{X})$ & + & India & $\begin{array}{c}\text { Asana and Makino } 1935 \text { as P. obscura } \\
\text { (Rambur, 1842) }\end{array}$ \\
\hline & & $-n-$ & + & India & Makino 1935 as P. obscura \\
\hline & & $-»-$ & + & India & Kichijo $1942 \mathrm{~b}$ as $P$. obscura \\
\hline & & $-"-$ & + & India & Dasgupta 1957 as $P$. obscura \\
\hline & & $-n-$ & + & India & Tyagi 1982 as $P$. obscura \\
\hline & & $-n-$ & + & India & Prasad and Thomas 1992 \\
\hline & & $-»-$ & + & India & Sandhu and Walia 1995 \\
\hline 349. & Pseudothemis zonata (Burmeister, 1839) & $24(22 \mathrm{~A}+$ neo-XY $)$ & - & Japan & Omura 1955 \\
\hline 350. & Pseudotramea prateri Fraser, 1920 & $25(24 \mathrm{~A}+\mathrm{X})$ & + & Nepal & Kiauta 1974,1975 \\
\hline 351. & Rhodopygia cardinalis (Erichson, 1848) & $25(24 \mathrm{~A}+\mathrm{X})$ & + & Bolivia & Cumming 1964 \\
\hline 352. & R. geijskesi Belle, 1964 & $25(24 \mathrm{~A}+\mathrm{X})$ & + & Surinam & Kiauta 1979a \\
\hline 353. & Rhodothemis rufa (Rambur, 1842) & $25(24 \mathrm{~A}+\mathrm{X})$ & + & India & Prasad and Thomas 1992 \\
\hline \multirow[t]{5}{*}{354.} & \multirow[t]{5}{*}{ Rhyothemis fuliginosa Selys, 1883} & $25(24 \mathrm{~A}+\mathrm{X})$ & + & Japan & Toyoshima and Hirai 1953 \\
\hline & & $-»-$ & + & Japan & Omura 1955 \\
\hline & & $-»-$ & + & Japan & Hirai 1956 \\
\hline & & $25(24 \mathrm{~A}+\mathrm{X})$ & + & Japan & Kiauta $1969 \mathrm{c}$ \\
\hline & & $23(22 \mathrm{~A}+\mathrm{X})$ & + & & \\
\hline \multirow[t]{2}{*}{355.} & \multirow[t]{2}{*}{ R. variegata (Linnaeus et Johansson, 1763) } & $25(24 \mathrm{~A}+\mathrm{X})$ & + & India & Ray Chaudhuri and Dasgupta 1949 \\
\hline & & $-»-$ & + & Nepal & Kiauta 1975 \\
\hline 356. & Scapanea frontalis (Burmeister, 1839) & $25(24 \mathrm{~A}+\mathrm{X})$ & + & Jamaica & Cumming 1964 \\
\hline 357. & Sympetrum commixtum (Selys, 1884) & $25(24 A+X)$ & - & India & Tyagi $1978 \mathrm{a}, \mathrm{b}, 1982$ \\
\hline 358. & S. corruptum (Hagen, 1861 & $25(24 \mathrm{~A}+\mathrm{X})$ & + & USA & $\begin{array}{c}\text { Cruden } 1968 \text { as Tarnetrum corruptum } \\
\text { (Hagen, 1861) }\end{array}$ \\
\hline & & $-»-$ & + & USA & Kiauta 1969a, c as T. corruptum \\
\hline 359. & S. costiferum (Hagen, 1861) & $25(24 \mathrm{~A}+\mathrm{X})$ & + & USA & Cruden 1968 \\
\hline 360. & S. croceolum (Selys, 1840) & $25(24 \mathrm{~A}+\mathrm{X})$ & + & Russia & Perepelov 2003 \\
\hline 361. & S. danae (Sulzer, 1776) & $25(24 \mathrm{~A}+\mathrm{X})$ & + & Former USSR & Makalowskaja 1940 \\
\hline & & $-»-$ & + & Finland & Oksala 1945 \\
\hline & & $-»-$ & + & USA & Cruden 1968 \\
\hline & & $-n-$ & + & Russia & Perepelov 2003 \\
\hline & & $-»-$ & + & Russia & Kuznetsova et al. 2018 \\
\hline 362. & S. eroticum (Selys, 1883) & $21(20 \mathrm{~A}+\mathrm{X})$ & - & Japan & Kichijo 1942b, c \\
\hline & & $-n-$ & - & Japan & Hirai 1956 \\
\hline & & $-n-$ & - & Japan & Kiauta $1969 \mathrm{c}$ \\
\hline 363. & S. flaveolum (Linnaeus, 1758) & $25(24 \mathrm{~A}+\mathrm{X})$ & + & Former USSR & Makalowskaja 1940 \\
\hline & & $-n-$ & + & Russia & Perepelov 2003 \\
\hline 364. & S. fonscolombii (Selys, 1840) & $25(24 \mathrm{~A}+\mathrm{X})$ & + & Russia & Perepelov 2003 \\
\hline 365. & S. frequens (Selys, 1883) & $23(22 \mathrm{~A}+\mathrm{X})$ & - & Japan & Oguma 1917, 1930 \\
\hline & & $-»-$ & - & Japan & Kichijo 1942a, b \\
\hline & & $-»-$ & - & Japan & Kiauta $1969 \mathrm{c}$ \\
\hline 366. & S. infuscatum (Selys, 1883) & $25(24 \mathrm{~A}+\mathrm{X})$ & + & Russia & Perepelov 2003 \\
\hline 367. & S. internum Montgomery, 1943 & $27(26 \mathrm{~A}+\mathrm{X})$ & + & Canada & Kiauta $1973 \mathrm{a}$ \\
\hline 368. & S. madidum (Hagen, 1861) & $25(24 \mathrm{~A}+\mathrm{X})$ & + & USA & Cruden 1968 \\
\hline
\end{tabular}




\begin{tabular}{|c|c|c|c|c|c|}
\hline \multicolumn{2}{|c|}{ Taxon } & \multirow{2}{*}{$\begin{array}{c}\begin{array}{c}\text { Karyotype } \\
\text { formula } 2 \mathbf{n}\end{array} \\
-n-\end{array}$} & \multirow{2}{*}{$\begin{array}{c}\begin{array}{c}\text { m-chromo } \\
\text { somes }\end{array} \\
+ \\
\end{array}$} & \multirow{2}{*}{$\begin{array}{l}\text { Country } \\
\text { Canada }\end{array}$} & \multirow{2}{*}{$\begin{array}{c}\text { References } \\
\text { Kiauta 1973a }\end{array}$} \\
\hline 368. & S. madidum (Hagen, 1861) & & & & \\
\hline 369. & S. meridionale (Selys, 1841) & $25(24 \mathrm{~A}+\mathrm{X})$ & + & Switzerland & Kiauta 1966 \\
\hline 370. & S. obtrusum (Hagen, 1867) & $25(24 \mathrm{~A}+\mathrm{X})$ & + & USA & Cruden 1968 \\
\hline 371. & S. parvulum Bartenev, 1912 & $25(24 \mathrm{~A}+\mathrm{X})$ & + & Japan & Kiauta $1968 \mathrm{c}$ \\
\hline \multirow[t]{6}{*}{372.} & \multirow[t]{6}{*}{ S. pedemontanum Müller in Allioni, 1766} & $25(24 \mathrm{~A}+\mathrm{X})$ & + & Japan & $\begin{array}{c}\text { Oguma } 1917,1930 \\
\text { (S. p. elatum (Selys, 1872)) }\end{array}$ \\
\hline & & $-n-$ & + & Japan & Kichijo 1942b (S.p. elatum) \\
\hline & & $-n-$ & + & Japan & Kiauta and Brink 1975 (S. p. elatum) \\
\hline & & $-n-$ & + & Switzerland & $\begin{array}{c}\text { Kiauta and Brink } 1975 \\
\text { (S.p. pedemontanum (Müller, 1766)) }\end{array}$ \\
\hline & & $-"-$ & + & Russia & $\begin{array}{l}\text { Perepelov et al. } 1998 \\
\text { (S.p.pedemontanum })\end{array}$ \\
\hline & & $-»-$ & + & Russia & Perepelov and Bugrov 2001b \\
\hline 373. & S. rubicundulum (Say, 1839) & $25(24 \mathrm{~A}+\mathrm{X})$ & + & USA & Cruden 1968 \\
\hline \multirow[t]{2}{*}{374.} & \multirow[t]{2}{*}{ S. sanguineum (Müller, 1764) } & $25(24 \mathrm{~A}+\mathrm{X})$ & + & Italy & Kiauta 1971a \\
\hline & & $-»-$ & + & Russia & Perepelov and Bugrov 2001b \\
\hline \multirow[t]{2}{*}{375.} & \multirow[t]{2}{*}{ S. semicinctum (Say, 1839) } & $25(24 \mathrm{~A}+\mathrm{X})$ & + & USA & Smith 1916 \\
\hline & & $-»-$ & + & USA & Cruden 1968 \\
\hline 376. & S. striolatum (Charpentier, 1840) & $25(24 \mathrm{~A}+\mathrm{X})$ & - & Luxembourg & Kiauta 1966 \\
\hline 377. & S. vicinum (Hagen, 1861) & $25(24 \mathrm{~A}+\mathrm{X})$ & + & USA & Cruden 1968 \\
\hline \multirow[t]{3}{*}{378.} & \multirow[t]{3}{*}{ S. vulgatum (Linnaeus, 1758) } & $25(24 \mathrm{~A}+\mathrm{X})$ & + & Netherland & Kiauta $1972 \mathrm{c}$ \\
\hline & & $-»-$ & + & Russia & Perepelov 2003 \\
\hline & & $-n-$ & + & Russia & Kuznetsova et al. 2018 \\
\hline \multirow[t]{2}{*}{379.} & \multirow[t]{2}{*}{ Tarnetrum illotum (Hagen, 1861) } & $25(24 \mathrm{~A}+\mathrm{X})$ & + & Jamaica & Cumming 1964 \\
\hline & & $-n-$ & + & USA & Cruden 1968 \\
\hline 380. & Tauriphila australis (Hagen, 1867) & $25(24 \mathrm{~A}+\mathrm{X})$ & + & Bolivia & Cumming 1964 \\
\hline 381. & T. azteca Calvert, 1906 & $25(24 \mathrm{~A}+\mathrm{X})$ & + & Mexico & Cruden 1968 \\
\hline 382. & T. risi Martin 1896 & $25(24 \mathrm{~A}+\mathrm{X})$ & + & $\begin{array}{l}\text { Argentina, } \\
\text { Uruguay }\end{array}$ & Mola and Agopian 1985 \\
\hline \multirow[t]{2}{*}{383.} & \multirow[t]{2}{*}{ Tholymis citrina Hagen, 1867} & $25(24 \mathrm{~A}+\mathrm{X})$ & + & Surinam & Kiauta 1979a \\
\hline & & $-n-$ & + & Brazil & Ferreira et al. 1979 \\
\hline \multirow[t]{3}{*}{384.} & \multirow[t]{3}{*}{ Th. tillagra (Fabricius, 1798) } & $25(24 \mathrm{~A}+\mathrm{X})$ & + & India & Prasad and Thomas 1992 \\
\hline & & $-n-$ & + & Nepal & Kiauta and Kiauta 1982 \\
\hline & & $-»-$ & + & Thailand & Kiauta and Kiauta 1983 \\
\hline 385. & Tramea abdominalis (Rambur, 1842) & $25(24 \mathrm{~A}+\mathrm{X})$ & - & Bolivia & Cumming 1964 \\
\hline \multirow[t]{4}{*}{386.} & \multirow[t]{4}{*}{ T. basilaris (Palisot de Beauvois, 1817) } & $25(24 \mathrm{~A}+\mathrm{X})$ & + & India & $\begin{array}{c}\text { Das } 1956 \text { (T. b. burmeisteri } \\
\text { (Kirby, 1889)) }\end{array}$ \\
\hline & & $-n-$ & + & India & Dasgupta 1957 (T. b. burmeisteri) \\
\hline & & $-n-$ & + & Nepal & $\begin{array}{c}\text { Kiauta and Kiauta } 1982 \\
\text { (T. b. burmeisteri) }\end{array}$ \\
\hline & & $-"-$ & + & India & $\begin{array}{l}\text { Prasad and Thomas } 1992 \\
\text { (T. b. burmeisteri) }\end{array}$ \\
\hline 387. & T. binotata (Rambur, 1842) & $25(24 \mathrm{~A}+\mathrm{X})$ & + & Surinam & Kiauta $1979 \mathrm{a}$ \\
\hline & & $-»-$ & - & Brazil & Ferreira et al. 1979 \\
\hline 388. & T. carolina (Linnaeus, 1763) & $25(24 \mathrm{~A}+\mathrm{X})$ & - & USA & Cumming 1964 \\
\hline & & $-»-$ & - & USA & Cruden 1968 \\
\hline 389. & T. cophysa (Hagen, 1867) & $25(24 \mathrm{~A}+\mathrm{X})$ & + & Bolivia & Cumming 1964 \\
\hline 390. & T. lacerata (Hagen, 1861) & $25(24 \mathrm{~A}+\mathrm{X})$ & - & USA & Cruden 1968 \\
\hline 391. & T. limbata (Desjardins, 1832) & $25(24 \mathrm{~A}+\mathrm{X})$ & + & India & Asana and Makino 1935 \\
\hline & & $-»-$ & + & India & Makino 1935 \\
\hline & & $-»-$ & + & India & Kichijo 1942b \\
\hline 392. & T. virginia (Rambur, 1842) & $25(24 \mathrm{~A}+\mathrm{X})$ & + & India & Oguma and Asana 1932 \\
\hline & & $-n-$ & + & India & Kichijo 1942b \\
\hline & & $-n-$ & + & India & Dasgupta 1957 \\
\hline 393. & $\begin{array}{l}\text { Trithemis annulata (Palisot de Beauvois, } \\
\text { 1805) }\end{array}$ & $25(24 \mathrm{~A}+\mathrm{X})$ & - & $\begin{array}{c}\text { Republic of South } \\
\text { Africa }\end{array}$ & Boyes et al. 1980 \\
\hline & & $-"-$ & + & Kenya & Wasscher 1985 \\
\hline 394. & T. arteriosa (Burmeister, 1839) & $25(24 \mathrm{~A}+\mathrm{X})$ & + & $\begin{array}{c}\text { Kingdom of } \\
\text { Eswatini (Former } \\
\text { Swaziland) }\end{array}$ & Boyes et al. 1980 \\
\hline
\end{tabular}




\begin{tabular}{|c|c|c|c|c|c|}
\hline \multicolumn{2}{|c|}{ Taxon } & \multirow{2}{*}{$\begin{array}{c}\begin{array}{c}\text { Karyotype } \\
\text { formula 2n } 0\end{array} \\
25(24 \mathrm{~A}+\mathrm{X})\end{array}$} & \multirow{2}{*}{$\begin{array}{c}\text { m-chromo } \\
\text { somes }\end{array}$} & \multirow{2}{*}{$\begin{array}{c}\text { Country } \\
\text { Burkina Faso } \\
\text { (Former Voltiac } \\
\text { Republic) } \\
\end{array}$} & \multirow{2}{*}{$\begin{array}{c}\text { References } \\
\text { Kiauta and Ochssée } 1979\end{array}$} \\
\hline 395. & T. atra Pinhey, 1961 & & & & \\
\hline \multirow[t]{3}{*}{396.} & \multirow[t]{3}{*}{ T. aurora (Burmeister, 1839) } & $25(24 \mathrm{~A}+\mathrm{X})$ & + & India & Oguma and Asana 1932 \\
\hline & & $-n-$ & + & Nepal & Kiauta 1975 \\
\hline & & $-n-$ & + & India & Tyagi 1982 \\
\hline 397. & T. dorsalis (Rambur, 1842) & $25(24 \mathrm{~A}+\mathrm{X})$ & + & $\begin{array}{c}\text { Kingdom of } \\
\text { Eswatini (Former } \\
\text { Swaziland) }\end{array}$ & Boyes et al. 1980 \\
\hline \multirow[t]{3}{*}{398.} & \multirow[t]{3}{*}{ T. festiva (Rambur, 1842) } & $25(24 \mathrm{~A}+\mathrm{X})$ & + & Nepal & Kiauta 1974,1975 \\
\hline & & $-»-$ & + & India & Tyagi 1982 \\
\hline & & $-»-$ & + & India & Prasad and Thomas 1992 \\
\hline 399. & T. furva Karsch, 1899 & $25(24 \mathrm{~A}+\mathrm{X})$ & + & Sudan & Wasscher 1985 \\
\hline 400. & T. imiata Pinhey, 1961 & $25(24 \mathrm{~A}+\mathrm{X})$ & - & $\begin{array}{l}\text { Burkina Faso } \\
\text { (Former Voltiac } \\
\text { Republic) }\end{array}$ & Kiauta and Ochssée 1979 \\
\hline \multirow[t]{2}{*}{401.} & \multirow[t]{2}{*}{ T. kirbyi Selys, 1891} & $25(24 \mathrm{~A}+\mathrm{X})$ & - & $\begin{array}{c}\text { Burkina Faso } \\
\text { (Former Voltiac } \\
\text { Republic) }\end{array}$ & $\begin{array}{c}\text { Kiauta and Ochssée } 1979 \\
\text { (T. k. ardens Gerstaecker, 1891) }\end{array}$ \\
\hline & & $-»-$ & + & Kenya & Wasscher 1985 (T. k. ardens) \\
\hline \multirow[t]{5}{*}{402.} & \multirow[t]{5}{*}{ T. pallidinervis (Kirby, 1889) } & $25(24 A+X)$ & + & India & Asana and Makino 1935 \\
\hline & & $-n-$ & + & India & Makino 1935 \\
\hline & & $-"-$ & + & India & Kichijo 1942b \\
\hline & & $-"-$ & + & India & Dasgupta 1957 \\
\hline & & $-"-$ & + & Philippines & Kiauta and Kiauta 1980b \\
\hline 403. & T. werneri $\mathrm{Ris}, 1912$ & $25(24 A+X)$ & + & Kenya & Wasscher 1985 \\
\hline \multirow[t]{2}{*}{404.} & \multirow[t]{2}{*}{ Uracis imbuta (Burmeister, 1839) } & $25(24 \mathrm{~A}+\mathrm{X})$ & + & Surinam & Kiauta 1979a \\
\hline & & $-n-$ & + & Brazil & Ferreira et al. 1979 \\
\hline \multirow[t]{2}{*}{405.} & \multirow[t]{2}{*}{ U. ovipositrix Calvert, 1909} & $25(24 \mathrm{~A}+\mathrm{X})$ & + & Surinam & Kiauta 1979 a \\
\hline & & $-»-$ & - & Brazil & Ferreira et al. 1979 \\
\hline 406. & Urothemis edwardsi (Selys, 1849) & $25(24 \mathrm{~A}+\mathrm{X})$ & + & Sudan & Wasscher 1985 \\
\hline \multirow[t]{4}{*}{407.} & \multirow[t]{4}{*}{ U. signata (Rambur, 1842) } & $25(24 \mathrm{~A}+\mathrm{X})$ & + & India & $\begin{array}{l}\text { Das } 1956 \text { (U. s. signata } \\
\text { (Rambur, 1842)) }\end{array}$ \\
\hline & & $-"-$ & + & India & Dasgupta 1957 (U. s. signata) \\
\hline & & $-"-$ & + & Nepal & Kiauta 1975 \\
\hline & & $-"-$ & + & India & Prasad and Thomas 1992 \\
\hline 408. & Zenithoptera fasciata (Linnaeus, 1758) & $25(24 \mathrm{~A}+\mathrm{X})$ & + & Surinam & Kiauta 1979a \\
\hline \multirow[t]{2}{*}{409.} & \multirow[t]{2}{*}{ Z. lanei Santos, 1941} & $25(24 \mathrm{~A}+\mathrm{X})$ & + & Surinam & Kiauta 1979 a \\
\hline & & $-"-$ & + & Brazil & Ferreira et al. 1979 \\
\hline 410. & Z. viola Ris, 1910 & $25(24 \mathrm{~A}+\mathrm{X})$ & + & Bolivia & Cumming 1964 \\
\hline 411. & Zygonyx iris Kirby, 1900 & $23(22 \mathrm{~A}+\mathrm{X})$ & + & Thailand & $\begin{array}{c}\text { Kiauta and Kiauta } 1983 \\
\text { (Z. i. malayanus (Laidlaw, 1902)) }\end{array}$ \\
\hline 412. & Z. torrida (Kirby, 1889) & $25(24 \mathrm{~A}+\mathrm{X})$ & + & India & Tyagi $1978 \mathrm{a}, \mathrm{b}$ \\
\hline 413. & Zyxomma petiolatum (Rambur, 1842) & $25(24 \mathrm{~A}+\mathrm{X})$ & + & India & Prasad and Thomas 1992 \\
\hline
\end{tabular}

\begin{tabular}{|c|c|c|c|c|c|}
\hline \multicolumn{6}{|c|}{ Chlorogomphidae } \\
\hline 414. & Watanabeopetalia atkinsoni (Selys, 1878) & $25(24 \mathrm{~A}+\mathrm{X})$ & + & India & Walia and Chahal 2019 \\
\hline \multicolumn{6}{|c|}{ Cordulegastridae } \\
\hline 415. & Anotogaster basalis Selys, 1854 & $23(22 \mathrm{~A}+\mathrm{X})$ & 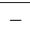 & India & Sandhu and Malhotra 1994b \\
\hline 416. & A. kuchenbeiseri (Förster, 1899) & $25(24 \mathrm{~A}+\mathrm{X})$ & + & China & Zhu and Wu 1986 \\
\hline \multirow[t]{4}{*}{417.} & \multirow[t]{4}{*}{ A. sieboldii (Selis, 1854) } & $25(24 \mathrm{~A}+\mathrm{X})$ & + & Japan & Oguma 1930 \\
\hline & & $-n-$ & + & Japan & Kichijo 1942a \\
\hline & & $-»-$ & + & Japan & Kiauta 1969a \\
\hline & & $-»-$ & + & Russia & Perepelov et al. 2001 \\
\hline \multirow[t]{3}{*}{418.} & \multirow[t]{3}{*}{ Cordulegaster boltoni (Donovan, 1807) } & $25(24 \mathrm{~A}+\mathrm{X})$ & + & Finland & Oksala 1939a, b \\
\hline & & $-»-$ & - & Austria & Kichijo $1942 \mathrm{a}$ \\
\hline & & $-»-$ & + & Sweden & Kiauta 1968d, e, 1969a \\
\hline 419. & C. brevistigma Selys, 1854 & $25(24 \mathrm{~A}+\mathrm{X})$ & + & India & Walia and Chahal 2019 \\
\hline 420. & C. diastatops (Selys, 1854) & $25(24 \mathrm{~A}+\mathrm{X})$ & + & USA & Cruden 1968 \\
\hline 421. & C. dorsalis Hagen, 1857 & $25(24 \mathrm{~A}+\mathrm{X})$ & + & USA & Cruden 1968 \\
\hline
\end{tabular}




\begin{tabular}{|c|c|c|c|c|c|}
\hline \multicolumn{2}{|c|}{ Taxon } & \multirow{2}{*}{$\begin{array}{c}\begin{array}{c}\text { Karyotype } \\
\text { formula } \mathbf{2 n}\end{array} \\
25(24 \mathrm{~A}+\mathrm{X}) \\
\end{array}$} & \multirow{2}{*}{$\begin{array}{c}\begin{array}{c}\text { m-chromo } \\
\text { somes }\end{array} \\
+ \\
\end{array}$} & \multirow{2}{*}{$\begin{array}{c}\text { Country } \\
\text { USA } \\
\end{array}$} & \multirow{2}{*}{$\begin{array}{c}\text { References } \\
\text { Cruden } 1968 \\
\end{array}$} \\
\hline 422. & C. maculata Selys, 1854 & & & & \\
\hline 423. & Neallogaster hermionae (Fraser, 1927) & $25(24 \mathrm{~A}+\mathrm{X})$ & + & Nepal & Kiauta and Kiauta 1976 \\
\hline \multicolumn{6}{|c|}{ ZYGOPTERA } \\
\hline \multicolumn{6}{|c|}{ LESTOIDEA } \\
\hline \multicolumn{6}{|c|}{ Lestidae } \\
\hline 424. & Austrolestes colensonis (White, 1846) & $25(24 \mathrm{~A}+\mathrm{X})$ & + & New Zealand & Jensen 1980 \\
\hline 425. & $\begin{array}{l}\text { Chalcolestes viridis (Van der Linden, } \\
1825 \text { ) }\end{array}$ & $25(24 \mathrm{~A}+\mathrm{X})$ & + & Netherlands & Kiauta 1969a \\
\hline 426. & Indolestes cyaneus (Selys, 1862) & $25(24 \mathrm{~A}+\mathrm{X})$ & + & Nepal & $\begin{array}{c}\text { Kiauta and Kiauta } 1976 \text { as I. cyanea } \\
\text { (Selys, 1862) }\end{array}$ \\
\hline 427. & Lestes barbarus (Fabricius, 1798) & $25(24 \mathrm{~A}+\mathrm{X})$ & + & Former Yugoslavia & Kiauta 1972a \\
\hline 428. & L. congener Hagen, 1861 & $25(24 \mathrm{~A}+\mathrm{X})$ & + & USA & Cruden 1968 \\
\hline 429. & L. disjunctus Selys, 1862 & $25(24 \mathrm{~A}+\mathrm{X})$ & - & USA & Cruden 1968 \\
\hline 430. & L. dorothea Fraser, 1924 & $25(24 A+X)$ & + & Nepal & Kiauta 1974,1975 \\
\hline \multirow[t]{2}{*}{431.} & \multirow[t]{2}{*}{ L. dryas Kirby, 1890} & $25(24 \mathrm{~A}+\mathrm{X})$ & - & USA & Cruden 1968 \\
\hline & & $-»-$ & + & Russia & Perepelov and Bugrov 2001b \\
\hline 432. & L. forcipatus Rambur, 1842 & $21(20 A+X)$ & - & USA & Cruden 1968 \\
\hline 433. & L. forficula Rambur, 1842 & $25(24 \mathrm{~A}+\mathrm{X})$ & + & Jamaica & Cumming 1964 \\
\hline 434. & L. paulistus Calvert, 1909 & $25(24 \mathrm{~A}+\mathrm{X})$ & + & Brazil & Souza Bueno 1982 \\
\hline 435. & L. rectangularis Say, 1839 & $25(24 \mathrm{~A}+\mathrm{X})$ & + & USA & Cruden 1968 \\
\hline 436. & L. similatrix McLachlan, 1895 & $25(24 \mathrm{~A}+\mathrm{X})$ & + & Madagascar & Kiauta $1969 b$ \\
\hline \multirow[t]{3}{*}{437.} & \multirow[t]{3}{*}{ L. sponsa (Hansemann, 1823) } & $25(24 \mathrm{~A}+\mathrm{X})$ & - & Former USSR & Makalowskaja 1940 \\
\hline & & $-»-$ & + & Japan & Kichijo 1941, 1942a, d, e \\
\hline & & $-n-$ & + & Russia & Perepelov and Bugrov 2001b \\
\hline 438. & L. stultus Hagen, 1861 & $25(24 \mathrm{~A}+\mathrm{X})$ & + & USA & Cruden 1968 \\
\hline 439. & L. vidua Hagen, 1861 & $25(24 A+X)$ & + & USA & Cumming 1964 \\
\hline 440. & L. vigilax Selys, 1862 & $19(18 \mathrm{~A}+\mathrm{X})$ & - & USA & Kiauta and Brink 1978 \\
\hline 441. & L. virens Charpentier, 1825 & $25(24 \mathrm{~A}+\mathrm{X})$ & + & Netherlands & $\begin{array}{c}\text { Kiauta 1969a } \\
\text { (L. v. vestalis Rambur, 1842) }\end{array}$ \\
\hline 442. & Sympecma fusca (Van der Linden, 1823) & $25(24 \mathrm{~A}+\mathrm{X})$ & + & Japan & Kichijo 1941, 1942d, e \\
\hline \multirow[t]{2}{*}{443.} & \multirow[t]{2}{*}{ S. paedisca (Brauer, 1877) } & $25(24 \mathrm{~A}+\mathrm{X})$ & + & Netherlands & $\begin{array}{l}\text { Kiauta and Kiauta-Brink } 1975 \\
\text { (S. annulata braueri (Bianchi, 1904)) }\end{array}$ \\
\hline & & $-n-$ & + & Russia & Perepelov 2003 (S. a. braueri) \\
\hline \multicolumn{6}{|c|}{ Synlestidae } \\
\hline 444. & Megalestes major Selys, 1862 & $25(24 \mathrm{~A}+\mathrm{X})$ & - & Nepal & Kiauta 1974,1975 \\
\hline \multicolumn{6}{|c|}{ Platystictoidea } \\
\hline \multicolumn{6}{|c|}{ Platystictidae } \\
\hline 445. & Drepanosticta sp. & $25(24 \mathrm{~A}+\mathrm{X})$ & - & Nepal & Kiauta and Kiauta 1976 \\
\hline 446. & Drepanosticta sp. & $25(24 \mathrm{~A}+\mathrm{X})$ & - & India & Tyagi $1978 \mathrm{a}, \mathrm{b}$ \\
\hline 447. & Palaemnema paulina (Drury, 1773) & $25(24 \mathrm{~A}+\mathrm{X})$ & + & Costa Rica & Cumming 1964 \\
\hline 448. & Protosticta sp. & $25(24 A+X)$ & - & Tailand & Kiauta and Kiauta 1983 \\
\hline \multicolumn{6}{|c|}{ CALOPTERYGoideA } \\
\hline \multicolumn{6}{|c|}{ Calopterygidae } \\
\hline 449. & $\begin{array}{l}\text { Atrocalopteryx atrata } \\
\text { (Selys, 1853) }\end{array}$ & $25(24 \mathrm{~A}+\mathrm{X})$ & + & Japan & $\begin{array}{c}\text { Oguma } 1930 \text { as Calopteryx atrata } \\
\text { Selys, } 1853\end{array}$ \\
\hline & & $-n-$ & + & Japan & Kichijo $1942 \mathrm{~d}$ as C. atrata \\
\hline & & $-n-$ & + & Japan & Omura 1957 as C. atrata \\
\hline 450. & Calopteryx aequabilis Say, 1839 & $25(24 \mathrm{~A}+\mathrm{X})$ & + & USA & Cruden 1968 \\
\hline 451. & C. cornelia (Selys, 1853) & $25(24 \mathrm{~A}+\mathrm{X})$ & + & Japan & $\begin{array}{c}\text { Oguma } 1930 \text { as Anaciagrion cornelia } \\
\text { (Selys, 1853) }\end{array}$ \\
\hline & & $-»-$ & + & Japan & Kichijo 1942 a as $A$. cornelia \\
\hline 452. & C. dimidiata Burmeister, 1839 & $25(24 \mathrm{~A}+\mathrm{X})$ & + & USA & Kiauta and Brink 1978 \\
\hline 453. & C. japonica Selys, 1869 & $25(24 \mathrm{~A}+\mathrm{X})$ & + & Japan & Kichijo 1942a \\
\hline & & $-n-$ & + & Japan & Hirai 1956 \\
\hline & & $-n-$ & + & Japan & Omura 1957 \\
\hline & & $-»-$ & + & Japan & Kiauta $1968 \mathrm{e}, \mathrm{f}$ \\
\hline 454. & C. maculata (Beauvois, 1805) & $25(24 \mathrm{~A}+\mathrm{X})$ & + & USA & Cumming 1964a \\
\hline & & $-»-$ & + & USA & Cruden 1968 \\
\hline 455. & C. splendens (Harris, 1780) & $25(24 \mathrm{~A}+\mathrm{X})$ & + & Turkey & $\begin{array}{c}\text { Kiauta } 1972 \mathrm{a} \\
\text { (C. s. amasina Bartenev, 1912) }\end{array}$ \\
\hline
\end{tabular}




\begin{tabular}{|c|c|c|c|c|c|}
\hline \multicolumn{2}{|c|}{ Taxon } & \multirow{2}{*}{$\begin{array}{c}\text { Karyotype } \\
\text { formula } 2 \mathbf{n} \delta \\
-"-\end{array}$} & \multirow{2}{*}{$\begin{array}{c}\begin{array}{c}\text { m-chromo } \\
\text { somes }\end{array} \\
+\end{array}$} & \multirow{2}{*}{$\begin{array}{c}\text { Country } \\
\text { Italy }\end{array}$} & \multirow{2}{*}{$\begin{array}{c}\text { References } \\
\text { Kiauta 1971a } \\
\text { (C. s. caprai Conci, 1956) }\end{array}$} \\
\hline 455. & C. splendens (Harris, 1780) & & & & \\
\hline & & $-"-$ & - & Former USSR & $\begin{array}{c}\text { Makalowskaja } 1940 \text { (C. s. splendens } \\
\text { (Harris, 1782)) }\end{array}$ \\
\hline & & $-n-$ & - & Finland & Oksala 1945 (C. s. splendens) \\
\hline & & $-n-$ & - & Germany & Kiauta 1969a, 1971b (C. s. splendens) \\
\hline & & $-n-$ & - & France & Kiauta 1973b (C.s. splendens) \\
\hline & & $-»-$ & - & Russia & Perepelov et al. 1998 (C. s. splendens) \\
\hline & & $-»-$ & + & Russia & Kuznetsova et al. 2020b \\
\hline \multirow[t]{10}{*}{456.} & \multirow[t]{10}{*}{ C. virgo (Linnaeus, 1758) } & $25(24 \mathrm{~A}+\mathrm{X})$ & + & \multirow[t]{2}{*}{ Spain } & \multirow{2}{*}{$\begin{array}{c}\text { Kiauta 1971b } \\
\text { (C. v. meridionalis Selys, 1873) }\end{array}$} \\
\hline & & $27(26 \mathrm{~A}+\mathrm{X})$ & + & & \\
\hline & & $25(24 \mathrm{~A}+\mathrm{X})$ & + & Slovenija & $\begin{array}{c}\text { Kiauta 1967a, 1968b, c (C. v. padana } \\
\text { Conci, 1956) }\end{array}$ \\
\hline & & $-"-$ & + & Austria & Kiauta 1967a, 1968b, c (C. v. padana) \\
\hline & & $-"-$ & - & Belgium & $\begin{array}{c}\text { Carnoy } 1885 \\
\text { (C. v. virgo (Linnaeus, 1758)) }\end{array}$ \\
\hline & & $-n-$ & + & Finland & Oksala 1939 (C.v. virgo) \\
\hline & & $-n-$ & + & Former USSR & Makalowskaja 1940 (C. v. virgo) \\
\hline & & $-»-$ & + & $\begin{array}{l}\text { Germany, } \\
\text { Luxembourg }\end{array}$ & Kiauta 1968e, $\mathrm{f}($ C. v. virgo $)$ \\
\hline & & $-n-$ & + & Netherlands & Kiauta $1972 \mathrm{c}(C . v$. virgo $)$ \\
\hline & & $-»-$ & + & Russia & Kuznetsova et al. 2020b \\
\hline \multirow[t]{2}{*}{457.} & \multirow[t]{2}{*}{ Hetaerina americana (Fabricius, 1798) } & $25(24 \mathrm{~A}+\mathrm{X})$ & + & USA & Cumming 1964 \\
\hline & & $-»-$ & & USA & Cruden 1968 \\
\hline 458. & H. charca Calvert, 1909 & $25(24 \mathrm{~A}+\mathrm{X})$ & + & Bolivia & Cumming 1964 \\
\hline \multirow[t]{2}{*}{459.} & \multirow[t]{2}{*}{ H. longipes (Hagen in Selys, 1853) } & $25(24 \mathrm{~A}+\mathrm{X})$ & + & Brazil & $\begin{array}{c}\text { Souza Bueno } 1982 \text { as } \\
\text { H. carnifex Hagen in Selys, } 1853\end{array}$ \\
\hline & & $-"-$ & + & Brazil & $\begin{array}{c}\text { Agopian and Mola } 1984 \text { as } \\
\text { H. carnifex }\end{array}$ \\
\hline \multirow[t]{4}{*}{460.} & \multirow[t]{4}{*}{ H. rosea Selys, 1853} & $27(26 \mathrm{~A}+\mathrm{X})$ & + & Bolivia & Cumming 1964 \\
\hline & & $-»-$ & + & Bolivia & Kiauta $1969 \mathrm{c}$ \\
\hline & & $25(24 \mathrm{~A}+\mathrm{X})$ & - & \multirow[t]{2}{*}{ Brazil } & \multirow[t]{2}{*}{ Ferreira et al. 1979} \\
\hline & & $27(26 \mathrm{~A}+\mathrm{X})$ & + & & \\
\hline 461. & H. sanguinea Selys, 1853 & $25(24 \mathrm{~A}+\mathrm{X})$ & - & Bolivia & Cumming 1964 \\
\hline \multirow[t]{2}{*}{462.} & \multirow[t]{2}{*}{ H. titia (Drury, 1773) } & $25(24 \mathrm{~A}+\mathrm{X})$ & + & USA & Cumming 1964 \\
\hline & & $-»-$ & + & Mexico & $\begin{array}{l}\text { Kiauta 1970a as H. tricolor } \\
\text { (Burmeister, 1839) }\end{array}$ \\
\hline 463. & H. vulnerata (Selys, 1853) & $25(24 \mathrm{~A}+\mathrm{X})$ & + & Mexico & Kiauta $1970 \mathrm{a}$ \\
\hline 464. & Matrona basilaris Selys, 1853 & $25(24 \mathrm{~A}+\mathrm{X})$ & - & Taiwan & Kiauta $1968 c$ \\
\hline \multirow[t]{2}{*}{465.} & \multirow[t]{2}{*}{ Mnais costalis Selys, 1869} & $25(24 \mathrm{~A}+\mathrm{X})$ & + & Japan & Oguma 1930 \\
\hline & & $-n-$ & + & Japan & Kichijo $1942 \mathrm{a}$ \\
\hline \multirow[t]{3}{*}{466.} & M. pruinosa Selys, 1853 & $25(24 \mathrm{~A}+\mathrm{X})$ & + & Japan & $\begin{array}{c}\text { Oguma } 1930 \text { as } \\
\text { M. strigata Selys, } 1853\end{array}$ \\
\hline & & $-n-$ & + & Japan & Kichijo 1942 a as $M$. strigata \\
\hline & & $-»-$ & + & Japan & Omura 1957 as $M$. strigata \\
\hline 467. & Neurobasis chinensis (Linnaeus, 1758) & $23(22 \mathrm{~A}+\mathrm{X})$ & - & Nepal & Kiauta 1975 (N. c. chinensis \\
\hline & & $25(24 \mathrm{~A}+\mathrm{X})$ & - & & (Linnaeus, 1758)) \\
\hline & & $23(22 \mathrm{~A}+\mathrm{X})$ & - & India & Tyagi 1978b (N. c. chinensis) \\
\hline & & $-»-$ & + & Nepal & $\begin{array}{c}\text { Kiauta and Kiauta } 1982 \\
(\text { N. c. chinensis })\end{array}$ \\
\hline & & $-»-$ & - & Thailand & $\begin{array}{l}\text { Kiauta and Kiauta } 1983 \\
\quad \text { (N. c. chinensis })\end{array}$ \\
\hline & & $-"-$ & + & India & $\begin{array}{c}\text { Walia and Sandhu } 2002 \\
\text { (N. c. chinensis })\end{array}$ \\
\hline & & $-n-$ & - & India & Walia et al. 2016 (N.c. chinensis) \\
\hline & & $-»-$ & - & India & $\begin{array}{c}\text { Walia and Katnoria } 2018 \\
(\text { N. c. chinensis })\end{array}$ \\
\hline 468. & Phaon iridipennis (Burmeister, 1839) & $25(24 \mathrm{~A}+\mathrm{X})$ & + & $\begin{array}{c}\text { Republic of South } \\
\text { Africa }\end{array}$ & Boyes et al. 1980 \\
\hline Chlo & rocyphidae & & & & \\
\hline 469. & Aristocypha fenestrella Rambur, 1842 & $23(22 \mathrm{~A}+\mathrm{X})$ & - & Thailand & $\begin{array}{c}\text { Kiauta and Kiauta } 1983 \text { as } \\
\text { Rhinocypha fenestrella Rambur, } 1842\end{array}$ \\
\hline
\end{tabular}




\begin{tabular}{|c|c|c|c|c|c|}
\hline \multicolumn{2}{|c|}{ Taxon } & \multirow{2}{*}{$\begin{array}{c}\begin{array}{c}\text { Karyotype } \\
\text { formula } \mathbf{2 n}\end{array} \\
23(22 \mathrm{~A}+\mathrm{X})\end{array}$} & \multirow{2}{*}{\begin{tabular}{c|}
$\begin{array}{c}\text { m-chromo } \\
\text { somes }\end{array}$ \\
+
\end{tabular}} & \multirow{2}{*}{$\begin{array}{c}\text { Country } \\
\text { India }\end{array}$} & \multirow{2}{*}{$\begin{array}{c}\text { References } \\
\text { Chatterjee and Kiauta } 1973 \text { as } \\
\text { Rhinocypha quadrimaculata Selys, } \\
1853\end{array}$} \\
\hline 470. & A. quadrimaculata (Selys, 1853) & & & & \\
\hline & & $-»-$ & + & Nepal & $\begin{array}{c}\text { Kiauta and Kiauta } 1982 \text { as } \\
\text { Rh. quadrimaculata }\end{array}$ \\
\hline \multirow[t]{2}{*}{471.} & \multirow[t]{2}{*}{ A. trifasciata (Selys, 1853) } & $23(22 \mathrm{~A}+\mathrm{X})$ & - & India & $\begin{array}{l}\text { Tyagi } 1978 \mathrm{a}, \mathrm{b} \text { as Rhinocypha } \\
\text { trifasciata Selys, } 1853\end{array}$ \\
\hline & & $-"-$ & + & Nepal & $\begin{array}{c}\text { Kiauta and Kiauta } 1982 \text { as } \\
R h . \text { trifasciata }\end{array}$ \\
\hline 472. & Heliocypha biforata (Selys, 1859) & $23(22 \mathrm{~A}+\mathrm{X})$ & - & India & $\begin{array}{c}\text { Tyagi } 1978 \text { a, b as Rhinocypha biforata } \\
\text { beesoni Selys, } 1859\end{array}$ \\
\hline 473. & H. biseriata (Selys, 1859) & $23(22 \mathrm{~A}+\mathrm{X})$ & - & Thailand & $\begin{array}{c}\text { Kiauta and Kiauta } 1983 \text { as } \\
\text { Rhinocypha b. biforata Selys, } 1859\end{array}$ \\
\hline \multirow[t]{2}{*}{474.} & \multirow[t]{2}{*}{ Libellago lineata (Burmeister, 1839) } & $23(22 \mathrm{~A}+\mathrm{X})$ & - & \multirow[t]{2}{*}{ India } & Walia et al. 2018 \\
\hline & & $25(24 \mathrm{~A}+\mathrm{X})$ & - & & (L. l. lineata (Burmeister, 1839)) \\
\hline \multirow[t]{2}{*}{475.} & \multirow[t]{2}{*}{ Paracypha unimaculata (Selys, 1879) } & $23(22 \mathrm{~A}+\mathrm{X})$ & + & Nepal & $\begin{array}{c}\text { Kiauta } 1974,1975 \text { as Rhinocypha } \\
\text { unimaculata Selys, } 1879\end{array}$ \\
\hline & & $-"-$ & + & Nepal & $\begin{array}{c}\text { Kiauta and Kiauta } 1982 \text { as } \\
\text { Rh. unimaculata }\end{array}$ \\
\hline \multirow[t]{2}{*}{476.} & \multirow[t]{2}{*}{ Rhinocypha colorata Selys, 1869} & $23(22 \mathrm{~A}+\mathrm{X})$ & - & \multirow[t]{2}{*}{ Philippines } & \multirow[t]{2}{*}{ Kiauta and Kiauta 1980b } \\
\hline & & $25(24 \mathrm{~A}+\mathrm{X})$ & - & & \\
\hline 477. & Vestalis gracilis (Rambur, 1842) & $25(24 \mathrm{~A}+\mathrm{X})$ & + & Thailand & Kiauta and Kiauta 1983 \\
\hline \multicolumn{6}{|c|}{ Polythoridae } \\
\hline 478. & Cora irene Ris, 1918 & $23(22 \mathrm{~A}+\mathrm{X})$ & - & Bolivia & Cumming 1964 \\
\hline 479. & Polythore boliviana (McLachlan, 1878) & $23(22 \mathrm{~A}+\mathrm{X})$ & - & Bolivia & Cumming 1964 \\
\hline \multicolumn{6}{|c|}{ Euphaeidae } \\
\hline 480. & Anisopleura comes Hagen, 1880 & $25(24 \mathrm{~A}+\mathrm{X})$ & + & Nepal & Kiauta and Kiauta 1976, 1982 \\
\hline \multirow[t]{2}{*}{481.} & \multirow[t]{2}{*}{ Bayadera indica (Selys, 1853) } & $25(24 \mathrm{~A}+\mathrm{X})$ & + & Nepal & Chatterjee and Kiauta 1973 \\
\hline & & $-n-$ & + & Nepal & Kiauta 1975 \\
\hline 482. & Euphaea guerini Rambur, 1842 & $25(24 \mathrm{~A}+\mathrm{X})$ & - & Thailand & Kiauta and Kiauta 1983 \\
\hline \multirow[t]{2}{*}{483.} & Epallage fatime (Charpentier, 1840) & $25(24 \mathrm{~A}+\mathrm{X})$ & - & Greece & Kiauta $1970 \mathrm{~b}$ \\
\hline & & $-n-$ & - & Greece & Chatterjee and Kiauta 1973 \\
\hline Mega & apodagrionidae & & & & \\
\hline 484. & Allopodagrion contortum (Selys, 1862) & $25(24 \mathrm{~A}+\mathrm{X})$ & + & Brazil & $\begin{array}{l}\text { Kiauta } 1972 \mathrm{~b} \text { as Megapodagrion } \\
\text { contortum (Selys, 1862) }\end{array}$ \\
\hline 485. & Teinopodagrion macropus (Selys, 1862) & $25(24 \mathrm{~A}+\mathrm{X})$ & - & Bolivia & $\begin{array}{c}\text { Cumming } 1964 \text { as Megapodagrion } \\
\text { macropus (Selys, 1862) }\end{array}$ \\
\hline 486. & T. setigerum (Selys, 1886) & $25(24 \mathrm{~A}+\mathrm{X})$ & - & Bolivia & $\begin{array}{c}\text { Cumming } 1964 \text { as Megapodagrion } \\
\text { setigerum Selys, } 1886\end{array}$ \\
\hline Heter & ragrionidae & & & & \\
\hline 487. & Heteragrion flavidorsum Calvert, 1909 & $25(24 \mathrm{~A}+\mathrm{X})$ & - & Bolivia & Cumming 1964 \\
\hline 488. & H. inca Calvert, 1909 & $25(24 \mathrm{~A}+\mathrm{X})$ & + & Bolivia & Cumming 1964 \\
\hline Philo & ogeniidae & & & & \\
\hline 489. & Philogenia carrillica Calvert, 1907 & $25(24 \mathrm{~A}+\mathrm{X})$ & + & Costa Rica & Cumming 1964 \\
\hline Hypo & olestidae & & & & \\
\hline 490. & Hypolestes clara (Calvert, 1891) & $17(16 \mathrm{~A}+\mathrm{X})$ & - & Jamaica & Cumming 1964 \\
\hline COEN & NAGRIONOIDEA & & & & \\
\hline Platyc & crnemididae & & & & \\
\hline 491. & Calicnemia miniata (Selys, 1886) & $25(24 A+X)$ & + & Nepal & Kiauta and Kiauta 1982 \\
\hline 492. & C. pulverulans (Selys, 1886) & $25(24 \mathrm{~A}+\mathrm{X})$ & - & Nepal & Kiauta 1975 \\
\hline 493. & Calicnemia sp. & $25(24 \mathrm{~A}+\mathrm{X})$ & - & Nepal & Kiauta 1975 \\
\hline 494. & Calicnemia sp. & $25(24 \mathrm{~A}+\mathrm{X})$ & - & India & Tyagi $1978 \mathrm{~b}$ \\
\hline 495. & Coeliccia chromothorax (Selys, 1891) & $25(24 \mathrm{~A}+\mathrm{X})$ & - & India & Walia and Devi 2020b \\
\hline 496. & C. bimaculata (Laidlaw, 1914) & $25(24 \mathrm{~A}+\mathrm{X})$ & - & India & Walia and Devi 2020b \\
\hline 497. & C. didyma (Selys, 1863) & $25(24 \mathrm{~A}+\mathrm{X})$ & - & India & Walia and Devi 2020b \\
\hline 498. & C. fraseri (Laidlaw, 1932) & $25(24 \mathrm{~A}+\mathrm{X})$ & - & India & Walia and Devi 2020b \\
\hline 499. & C. renifera (Selys, 1886) & $25(24 \mathrm{~A}+\mathrm{X})$ & - & Nepal & Kiauta 1974,1975 \\
\hline & & $-»-$ & - & India & Walia and Devi 2020b \\
\hline 500. & Copera annulata (Selys, 1863) & $25(24 \mathrm{~A}+\mathrm{X})$ & + & Japan & Kichijo 1941, 1942a, c \\
\hline & & $-»-$ & + & India & Dasgupta 1957 \\
\hline & & $-n-$ & - & Thailand & Kiauta and Kiauta 1983 \\
\hline & & $-n-$ & + & India & Walia and Devi 2018 \\
\hline
\end{tabular}




\begin{tabular}{|c|c|c|c|c|c|}
\hline \multicolumn{2}{|c|}{ Taxon } & \multirow{2}{*}{$\begin{array}{c}\begin{array}{c}\text { Karyotype } \\
\text { formula 2n }\end{array} \\
25(24 \mathrm{~A}+\mathrm{X})\end{array}$} & \multirow{2}{*}{$\begin{array}{c}\begin{array}{c}\text { m-chromo } \\
\text { somes }\end{array} \\
- \\
\end{array}$} & \multirow{2}{*}{$\begin{array}{c}\text { Country } \\
\text { India }\end{array}$} & \multirow{2}{*}{$\begin{array}{c}\text { References } \\
\text { Tyagi 1978a, b }\end{array}$} \\
\hline 501. & C. marginipes (Rambur, 1842) & & & & \\
\hline & & $-»-$ & - & Thailand & Kiauta and Kiauta 1983 \\
\hline & & $-n-$ & + & India & Walia and Devi 2018 \\
\hline \multirow[t]{2}{*}{502.} & \multirow[t]{2}{*}{ C. vittata (Selys, 1863) } & $25(24 A+X)$ & + & India & Walia and Devi 2018 \\
\hline & & $-»-$ & + & India & $\begin{array}{c}\text { Walia and Devi } 2018 \\
\text { (C. v. assamensis (Laidlaw, 1914)) }\end{array}$ \\
\hline 503. & $\begin{array}{l}\text { Disparoneura quadrimaculata (Rambur, } \\
\text { 1842) }\end{array}$ & $25(24 \mathrm{~A}+\mathrm{X})$ & - & India & Walia and Devi 2020a \\
\hline 504. & Esme cyaneovittata Fraser, 1922 & $25(24 \mathrm{~A}+\mathrm{X})$ & - & India & Walia and Devi 2020a \\
\hline 505. & E. longistyla Fraser, 1931 & $25(24 A+X)$ & - & India & Walia and Devi 2020a \\
\hline 506. & Onychargia atrocyana (Selys, 1865) & $25(24 \mathrm{~A}+\mathrm{X})$ & - & Thailand & Kiauta and Kiauta 1983 \\
\hline \multirow[t]{3}{*}{507.} & \multirow[t]{3}{*}{ Platycnemis pennipes (Pallas, 1771) } & $25(24 \mathrm{~A}+\mathrm{X})$ & - & Finland & Oksala 1945 \\
\hline & & $-»-$ & - & Italy & Kiauta 1971a \\
\hline & & $-n-$ & - & Russia & Perepelov and Bugrov 2001b \\
\hline 508. & Prodasineura autumnalis (Fraser, 1922) & $25(24 \mathrm{~A}+\mathrm{X})$ & + & Thailand & Kiauta and Kiauta 1983 \\
\hline 509. & P. nigra (Fraser, 1922) & $25(24 \mathrm{~A}+\mathrm{X})$ & - & India & Walia and Devi 2020a \\
\hline 510. & P. verticalis (Selys, 1860) & $25(24 \mathrm{~A}+\mathrm{X})$ & - & India & Walia and Devi 2020a \\
\hline 511. & Prodasineura sp.1 & $25(24 \mathrm{~A}+\mathrm{X})$ & - & Thailand & Kiauta and Kiauta 1983 \\
\hline 512. & Prodasineura sp. 2 & $25(24 \mathrm{~A}+\mathrm{X})$ & - & Thailand & Kiauta and Kiauta 1983 \\
\hline \multicolumn{6}{|c|}{ Coenagrionidae } \\
\hline 513. & Acanthagrion ascendens Calvert, 1909 & $27(26 \mathrm{~A}+\mathrm{X})$ & + & Bolivia & Cumming 1964 \\
\hline 514. & A. chacoense Calvert, 1909 & $27(26 \mathrm{~A}+\mathrm{X})$ & + & Bolivia & Cumming 1964 \\
\hline \multirow[t]{2}{*}{515.} & \multirow[t]{2}{*}{ A. gracile (Rambur, 1842) } & $27(26 \mathrm{~A}+\mathrm{X})$ & - & Surinam & $\begin{array}{c}\text { Kiauta } 1979 \mathrm{a} \\
\text { (A. g. minarum Selys, 1876) }\end{array}$ \\
\hline & & $-n-$ & - & Brazil & $\begin{array}{c}\text { Ferreira et al. } 1979 \\
\text { (A. g. minarum Selys, 1876) }\end{array}$ \\
\hline 516. & Aeolagrion inca Selys, 1876 & $27(26 \mathrm{~A}+\mathrm{X})$ & - & Bolivia & $\begin{array}{l}\text { Cumming } 1964 \text { as } A \text {. foliaceum } \\
\text { (Sjöstedt, 1918) }\end{array}$ \\
\hline 517. & Agriocnemis clauseni Fraser, 1922 & $27(26 \mathrm{~A}+\mathrm{X})$ & + & India & Tyagi $1978 \mathrm{a}, \mathrm{b}$ \\
\hline \multirow[t]{2}{*}{518.} & \multirow[t]{2}{*}{ A. femina (Brauer, 1868) } & $27(26 \mathrm{~A}+\mathrm{X})$ & - & Philippines & Kiauta and Kiauta 1980b \\
\hline & & $-»-$ & + & Thailand & Kiauta and Kiauta 1983 \\
\hline \multirow[t]{2}{*}{519.} & \multirow[t]{2}{*}{ A. pygmaea (Rambur, 1842) } & $27(26 \mathrm{~A}+\mathrm{X})$ & - & India & Tyagi $1978 \mathrm{~b}$ \\
\hline & & $-n-$ & + & Thailand & Kiauta and Kiauta 1983 \\
\hline 520. & Amphiagrion abbreviatum (Selys, 1876) & $27(26 \mathrm{~A}+\mathrm{X})$ & - & USA & Cruden 1968 \\
\hline 521. & Amphiallagma parvum (Selys, 1876) & $27(26 \mathrm{~A}+\mathrm{X})$ & + & India & $\begin{array}{l}\text { Handa and Kochhar } 1985 \text { as } \\
\text { Enallagma parvum Selys, } 1876\end{array}$ \\
\hline 522. & Argia apicalis (Say, 1839) & $37(36 \mathrm{~A}+\mathrm{X})$ & - & USA & Kiauta and Kiauta 1980b \\
\hline \multirow[t]{4}{*}{523.} & \multirow[t]{4}{*}{ A. fumipennis (Burmeister, 1839) } & $27(26 \mathrm{~A}+\mathrm{X})$ & - & USA & $\begin{array}{l}\text { Kiauta and Kiauta 1980c } \\
\text { (A. f. atra Gloyd, 1968) }\end{array}$ \\
\hline & & $-n-$ & - & USA & $\begin{array}{c}\text { Kiauta and Brink } 1978 \\
\text { (A.f. fumipennis (Burmeister, 1839)) }\end{array}$ \\
\hline & & $-»-$ & - & USA & $\begin{array}{l}\text { Kiauta and Kiauta 1980c } \\
\text { (A.f. fumipennis) }\end{array}$ \\
\hline & & $-"-$ & + & Canada & $\begin{array}{l}\text { Kiauta and Kiauta 1980c } \\
\text { (A.f. violaced (Hagen, 1861)) }\end{array}$ \\
\hline \multirow[t]{2}{*}{524.} & \multirow[t]{2}{*}{ A. funebris (Hagen, 1861) } & $27(26 \mathrm{~A}+\mathrm{X})$ & - & USA & Kiauta $1972 \mathrm{~b}$ \\
\hline & & $28(26 \mathrm{~A}+\mathrm{XX})^{*}$ & - & Mexico & Kiauta and Kiauta 1980c \\
\hline 525. & A. immunda (Hagen, 1861) & $27(26 \mathrm{~A}+\mathrm{X})$ & - & USA & Kiauta and Kiauta 1980c \\
\hline \multirow[t]{2}{*}{526.} & A. moesta (Hagen, 1861) & $25(24 \mathrm{~A}+\mathrm{X})$ & - & Canada & Kiauta 1978 \\
\hline & & $-»-$ & - & USA & Kiauta and Kiauta 1980c \\
\hline 527. & A. nahuana Calvert, 1902 & $25(24 \mathrm{~A}+\mathrm{X})$ & - & USA & Kiauta and Kiauta 1980c \\
\hline 528. & A. sedula (Hagen, 1861) & $27(26 \mathrm{~A}+\mathrm{X})$ & - & USA & Cruden 1968 \\
\hline & & $-»-$ & - & USA & Kiauta and Kiauta 1980c \\
\hline 529. & A. tibialis (Rambur, 1842) & $37(36 \mathrm{~A}+\mathrm{X})$ & - & USA & Kiauta and Kiauta 1980c \\
\hline 530. & A. translata Hagen, 1865 & $25(24 \mathrm{~A}+\mathrm{X})$ & + & USA & Kiauta and Kiauta 1980c \\
\hline 531. & A. violacea (Hagen, 1861) & $27(26 \mathrm{~A}+\mathrm{X})$ & - & USA & Cruden 1968 \\
\hline 532. & A. vivida (Hagen, 1861) & $27(26 A+X)$ & - & USA & Cruden 1968 \\
\hline 533. & Ceriagrion auranticum Fraser, 1922 & $27(26 A+X)$ & + & Thailand & $\begin{array}{l}\text { Kiauta and Kiauta } 1983 \text { as } \\
\text { C. latericium Lieftinck, } 1951\end{array}$ \\
\hline 534. & C. azureum (Selys, 1891) & $27(26 \mathrm{~A}+\mathrm{X})$ & - & Nepal & Kiauta 1974,1975 \\
\hline 535. & C. cerinomelas Lieftinck, 1927 & $27(26 A+X)$ & - & Nepal & Kiauta 1974,1975 \\
\hline
\end{tabular}




\begin{tabular}{|c|c|c|c|c|c|}
\hline \multicolumn{2}{|c|}{ Taxon } & \multirow{2}{*}{$\begin{array}{c}\begin{array}{c}\text { Karyotype } \\
\text { formula } \mathbf{2 n}\end{array} \\
27(26 \mathrm{~A}+\mathrm{X}) \\
\end{array}$} & \multirow{2}{*}{$\begin{array}{c}\begin{array}{c}\text { m-chromo } \\
\text { somes }\end{array} \\
+ \\
\end{array}$} & \multirow{2}{*}{$\begin{array}{c}\text { Country } \\
\text { India }\end{array}$} & \multirow{2}{*}{$\begin{array}{c}\text { References } \\
\text { Dasgupta } 1957\end{array}$} \\
\hline 536. & C. cerinorubellum (Brauer, 1866) & & & & \\
\hline & & $-n-$ & + & India & Prasad and Thomas 1992 \\
\hline \multirow[t]{5}{*}{537.} & \multirow[t]{5}{*}{ C. coromandelianum (Fabricius, 1798) } & $27(26 \mathrm{~A}+\mathrm{X})$ & + & India & Ray Chaudhuri and Dasgupta 1949 \\
\hline & & $-»-$ & + & India & Srivastava and Das 1953 \\
\hline & & $-n-$ & + & India & Das 1956 \\
\hline & & $-n-$ & + & Nepal & Kiauta and Kiauta 1982 \\
\hline & & $-n-$ & + & India & Prasad and Thomas 1992 \\
\hline 538. & C. fallax Ris, 1914 & $27(26 \mathrm{~A}+\mathrm{X})$ & + & $\begin{array}{c}\text { Republic of South } \\
\text { Africa }\end{array}$ & Dasgupta 1957 \\
\hline 539. & C. glabrum (Burmeister, 1839) & $27(26 \mathrm{~A}+\mathrm{X})$ & - & $\begin{array}{c}\text { Kingdom of } \\
\text { Eswatini (Former } \\
\text { Swaziland) } \\
\end{array}$ & Boyes et al. 1980 \\
\hline \multirow[t]{3}{*}{540.} & \multirow[t]{3}{*}{ C. rubiae Laidlaw, 1916} & $27(26 \mathrm{~A}+\mathrm{X})$ & + & India & Asana and Makino 1935 \\
\hline & & $-»-$ & + & India & Makino 1935 \\
\hline & & $-n-$ & + & India & Kichijo 1942a \\
\hline 541. & C. tenellum (Villers, 1789) & $27(26 \mathrm{~A}+\mathrm{X})$ & + & Italy & $\begin{array}{c}\text { Kiauta 1971a } \\
\text { (C.t.tenellum (Villers, 1789)) }\end{array}$ \\
\hline 542. & Chromagrion conditum (Hagen, 1876) & $27(26 \mathrm{~A}+\mathrm{X})$ & - & USA & Cruden 1968 \\
\hline \multirow[t]{2}{*}{543.} & \multirow[t]{2}{*}{ Coenagrion armatum (Charpentier, 1840) } & $27(26 \mathrm{~A}+\mathrm{X})$ & - & Finland & Oksala 1939a \\
\hline & & $-»-$ & - & Former USSR & Makalowskaja 1940 \\
\hline \multirow[t]{2}{*}{544.} & \multirow[t]{2}{*}{ C. hastulatum (Charpentier, 1825) } & $27(26 \mathrm{~A}+\mathrm{X})$ & - & Former USSR & Makalowskaja 1940 \\
\hline & & $-n-$ & - & Russia & Perepelov and Bugrov 2001b \\
\hline 545. & C. hylas (Trybom, 1889) & $27(26 \mathrm{~A}+\mathrm{X})$ & - & Austria & $\begin{array}{l}\text { Kiauta and Kiauta 1991 } \\
\text { (C. h. freyi (Bilek, 1954)) }\end{array}$ \\
\hline 546. & C. lunulatum (Charpentier, 1840) & $27(26 A+X)$ & + & Russia & Perepelov and Bugrov 2001b \\
\hline \multirow[t]{3}{*}{547.} & \multirow[t]{3}{*}{ C. pulchellum (Vander Linden, 1823) } & $27(26 \mathrm{~A}+\mathrm{X})$ & - & Former USSR & Makalowskaja 1940 \\
\hline & & $-»-$ & - & Netherlands & Kiauta $1969 \mathrm{c}$ \\
\hline & & $-»-$ & + & Russia & Kuznetsova et al. 2020b \\
\hline 548. & C. puella (Linnaeus, 1758) & $27(26 \mathrm{~A}+\mathrm{X})$ & + & Russia & Kuznetsova et al. 2020b \\
\hline 549. & C. resolutum (Hagen, 1876) & $27(26 \mathrm{~A}+\mathrm{X})$ & - & USA & Cruden 1968 \\
\hline 550. & Coenagrion sp. & $27(26 \mathrm{~A}+\mathrm{X})$ & + & Japan & Kichijo 1941, 1942d, e \\
\hline 551. & Diceratobasis macrogaster (Selys, 1875) & $27(26 \mathrm{~A}+\mathrm{X})$ & + & Jamaica & Cumming 1964 \\
\hline 552. & Enallagma aspersum (Hagen, 1861) & $27(26 \mathrm{~A}+\mathrm{X})$ & - & USA & Cruden 1968 \\
\hline 553. & E. boreale Selys, 1875 & $27(26 \mathrm{~A}+\mathrm{X})$ & - & USA & Cruden 1968 \\
\hline 554. & E. carunculatum Morse, 1895 & $27(26 \mathrm{~A}+\mathrm{X})$ & - & USA & Cruden 1968 \\
\hline 555. & E. circulatum Selys, 1883 & $27(26 A+X)$ & + & Russia & Perepelov and Bugrov 2001b \\
\hline 556. & E. civile (Hagen, 1861) & $27(26 \mathrm{~A}+\mathrm{X})$ & - & USA & Cruden 1968 \\
\hline \multirow[t]{7}{*}{557.} & \multirow[t]{7}{*}{ E. cyathigerum (Charpentier, 1840) } & $27(26 \mathrm{~A}+\mathrm{X})$ & - & Finland & Oksala 1939a, 1945 \\
\hline & & $-»-$ & - & Former USSR & Makalowskaja 1940 \\
\hline & & $-»-$ & + & USA & Brink and Kiauta 1964 \\
\hline & & $27(26 \mathrm{~A}+\mathrm{X})$ & - & \multirow[t]{2}{*}{ USA } & \multirow[t]{2}{*}{ Cruden 1968} \\
\hline & & $29(28 \mathrm{~A}+\mathrm{X})$ & - & & \\
\hline & & $27(26 \mathrm{~A}+\mathrm{X})$ & + & \multirow[t]{2}{*}{ Netherlands } & Kiauta 1969a, c \\
\hline & & $29(28 \mathrm{~A}+\mathrm{X})$ & + & & \\
\hline 558. & E. ebrium (Hagen, 1861) & $27(26 A+X)$ & - & USA & Cruden 1968 \\
\hline 559. & E. praevarum (Hagen, 1861$)$ & $27(26 \mathrm{~A}+\mathrm{X})$ & - & USA & Cruden 1968 \\
\hline 560. & Erythromma lindeni (Selys, 1840) & $27(26 \mathrm{~A}+\mathrm{X})$ & + & Italy & Kiauta 1971a \\
\hline 561. & E. najas (Hansemann, 1823) & $27(26 \mathrm{~A}+\mathrm{X})$ & - & Finland & Oksala 1939a \\
\hline & & $-»-$ & - & Former USSR & Makalowskaja 1940 \\
\hline & & $-»-$ & - & Netherlands & Kiauta 1969a \\
\hline & & $-»-$ & - & Russia & Perepelov and Bugrov 2001b \\
\hline & & $-»-$ & + & Russia & Kuznetsova et al. 2020b \\
\hline 562. & Homeoura chelifera (Selys, 1876) & $27(26 \mathrm{~A}+\mathrm{X})$ & + & Surinam & $\begin{array}{c}\text { Kiauta 1979a as Enallagma cheliferum } \\
\text { (Selys, 1876) }\end{array}$ \\
\hline & & $-»-$ & + & Brazil & Ferreira et al. 1979 as E. cheliferum \\
\hline 563. & Ischnura aurora (Brauer, 1865) & $27(26 \mathrm{~A}+\mathrm{X})$ & - & Nepal & Kiauta 1974,1975 \\
\hline & & $-»-$ & - & India & Handa and Kochhar 1985 \\
\hline 564. & I. capreola (Hagen, 1861) & $27(26 \mathrm{~A}+\mathrm{X})$ & - & Bolivia & $\begin{array}{c}\text { Cumming } 1964 \text { as Ceratura capreola } \\
\text { (Hagen, 1861) }\end{array}$ \\
\hline
\end{tabular}




\begin{tabular}{|c|c|c|c|c|c|}
\hline \multicolumn{2}{|c|}{ Taxon } & \multirow{2}{*}{$\begin{array}{c}\begin{array}{c}\text { Karyotype } \\
\text { formula } \mathbf{2 n}\end{array} \\
27(26 \mathrm{~A}+\mathrm{X}) \\
\end{array}$} & \multirow{2}{*}{$\begin{array}{c}\begin{array}{c}\text { m-chromo } \\
\text { somes }\end{array} \\
-\end{array}$} & \multirow{2}{*}{$\begin{array}{c}\text { Country } \\
\text { USA }\end{array}$} & \multirow{2}{*}{$\begin{array}{c}\text { References } \\
\text { Cruden } 1968 \\
\end{array}$} \\
\hline 565. & I. cervula Selys, 1876 & & & & \\
\hline 566. & I. denticollis (Burmeister, 1839) & $27(26 \mathrm{~A}+\mathrm{X})$ & - & USA & Cruden 1968 \\
\hline \multirow[t]{3}{*}{567.} & \multirow[t]{3}{*}{ I. elegans (Van der Linden, 1823) } & $27(26 \mathrm{~A}+\mathrm{X})$ & - & Finland & Oksala 1939a, 1945 \\
\hline & & $-n-$ & - & Netherlands & Kiauta 1969 a \\
\hline & & $-n-$ & - & Russia & Perepelov 2003 \\
\hline 568. & I. fluviatilis Selys, 1876 & $27(26 \mathrm{~A}+\mathrm{X})$ & - & Bolivia & Cumming 1964 \\
\hline 569. & I. forcipata Morton, 1907 & $27(26 \mathrm{~A}+\mathrm{X})$ & - & Nepal & Kiauta 1974,1975 \\
\hline 570. & I. nursei (Morton, 1907) & $25(24 \mathrm{~A}+\mathrm{X})$ & + & India & $\begin{array}{l}\text { Tyagi } 1978 \mathrm{~b} \text { as Rhodischnura nursei } \\
\text { (Morton, 1907) }\end{array}$ \\
\hline 571. & I. pumilio (Charpentier, 1825) & $27(26 \mathrm{~A}+\mathrm{X})$ & + & Netherlands & Kiauta 1979b \\
\hline 572. & I. perparva Selys, 1876 & $27(26 \mathrm{~A}+\mathrm{X})$ & - & USA & Cruden 1968 \\
\hline 573. & I. ramburii (Selys, 1850) & $27(26 \mathrm{~A}+\mathrm{X})$ & + & USA & Kiauta and Brink 1978 \\
\hline 574. & I. rufostigma Selys, 1876 & $27(26 \mathrm{~A}+\mathrm{X})$ & - & Nepal & $\begin{array}{c}\text { Kiauta 1974, } 1975 \\
\text { (I. r. annandalei Laidlaw, 1919) }\end{array}$ \\
\hline \multirow[t]{6}{*}{575.} & \multirow[t]{6}{*}{ I. senegalensis (Rambur, 1842) } & $27(26 \mathrm{~A}+\mathrm{X})$ & + & Japan & Kichijo 1941, 1942d, e \\
\hline & & $-»-$ & + & India & Dasgupta 1957 \\
\hline & & $-n-$ & + & Ethiopia & Kiauta $1969 \mathrm{~b}$ \\
\hline & & $-n-$ & + & Philippines & Kiauta and Kiauta 1980b \\
\hline & & $-»-$ & - & Thailand & Kiauta and Kiauta 1983 \\
\hline & & $-»-$ & + & India & Prasad and Thomas 1992 \\
\hline 576. & I. verticalis (Say, 1839) & $27(26 \mathrm{~A}+\mathrm{X})$ & - & USA & Cruden 1968 \\
\hline 577. & I. ultima Ris, 1908 & $27(26 \mathrm{~A}+\mathrm{X})$ & - & Bolivia & Cumming 1964 \\
\hline 578. & Leptagrion macrurum (Burmeister, 1839) & $30(28 \mathrm{~A}+$ neo-XY $)$ & - & Brazil & Kiauta $1971 \mathrm{c}, 1972 \mathrm{~d}$ \\
\hline 579. & Mecistogaster. sp. 1 & $29(28 \mathrm{~A}+\mathrm{X})$ & + & Bolivia & Cumming 1964 \\
\hline 580. & Mecistogaster sp. 2 & $12(10 \mathrm{~A}+$ neo-XY $)$ & - & Bolivia & Cumming 1964 \\
\hline 581. & Megalagrion oahuense (Blackburn, 1884) & $27(26 \mathrm{~A}+\mathrm{X})$ & + & Hawaii & Kiauta 1969b \\
\hline 582. & Mortonagrion selenion (Ris, 1916) & $27(26 \mathrm{~A}+\mathrm{X})$ & + & Japan & Kichijo 1941, 1942a, d, e \\
\hline 583. & Nehalennia irene (Hagen, 1861) & $27(26 \mathrm{~A}+\mathrm{X})$ & - & USA & Cruden 1968 \\
\hline 584. & N. speciosa (Charpentier, 1840) & $28(26 \mathrm{~A}+\mathrm{XX})^{*}$ & - & Finland & Oksala 1945 \\
\hline 585. & Oxyagrion hempeli Calvert, 1909 & $27(26 \mathrm{~A}+\mathrm{X})$ & - & Brazil & Souza Bueno 1982 \\
\hline \multirow[t]{2}{*}{586.} & \multirow[t]{2}{*}{ O. terminale Selys, 1876} & $27(26 \mathrm{~A}+\mathrm{X})$ & - & Surinam & Kiauta $1979 \mathrm{a}$ \\
\hline & & $-»-$ & - & Brazil & Ferreira et al. 1979 \\
\hline 587. & Paracercion hieroglyphicum (Brauer, 1865) & $27(26 \mathrm{~A}+\mathrm{X})$ & + & Japan & $\begin{array}{c}\text { Kichijo 1941, 1942d, e as Coenagrion } \\
\text { hieroglyphicum (Brauer, 1865) }\end{array}$ \\
\hline 588. & P. malayanum (Selys, 1876) & $27(26 \mathrm{~A}+\mathrm{X})$ & + & Nepal & Kiauta 1974,1975 \\
\hline 589. & Proischnura subfurcata (Selys, 1876) & $27(26 \mathrm{~A}+\mathrm{X})$ & - & Kenya & $\begin{array}{l}\text { Wasscher } 1985 \text { as Enallagma } \\
\text { subfurcatum Selys, } 1876\end{array}$ \\
\hline 590. & Pseudagrion acaciae Förster, 1906 & $27(26 \mathrm{~A}+\mathrm{X})$ & + & $\begin{array}{c}\text { Republic of South } \\
\text { Africa }\end{array}$ & Boyes et al. 1980 \\
\hline 591. & P. australasiae Selys, 1876 & $27(26 \mathrm{~A}+\mathrm{X})$ & + & India & Dasgupta 1957 \\
\hline 592. & P. decorum (Rambur, 1842) & $27(26 \mathrm{~A}+\mathrm{X})$ & + & India & Dasgupta 1957 \\
\hline 593. & P. kersteni (Gerstaker, 1869) & $27(26 \mathrm{~A}+\mathrm{X})$ & - & $\begin{array}{c}\text { Kingdom of } \\
\text { Eswatini (Former } \\
\text { Swaziland) } \\
\end{array}$ & Boyes et al. 1980 \\
\hline \multirow[t]{2}{*}{594.} & \multirow[t]{2}{*}{ P. microcephalum (Rambur, 1842) } & $27(26 \mathrm{~A}+\mathrm{X})$ & + & India & Dasgupta 1957 \\
\hline & & $-n-$ & + & Philippines & Kiauta and Kiauta 1980b \\
\hline 595. & P. pruinosum (Burmeister, 1839) & $27(26 \mathrm{~A}+\mathrm{X})$ & + & Thailand & Kiauta and Kiauta 1983 \\
\hline \multirow[t]{3}{*}{596.} & \multirow[t]{3}{*}{ P. rubripes (Selys, 1876) } & $27(26 \mathrm{~A}+\mathrm{X})$ & + & India & Dasgupta 1957 \\
\hline & & $-»-$ & + & Philippines & Kiauta and Kiauta $1980 \mathrm{~b}$ \\
\hline & & $-»-$ & + & Thailand & Kiauta and Kiauta 1983 \\
\hline 597. & P. salisburyense Ris, 1921 & $27(26 A+X)$ & + & $\begin{array}{c}\text { Kingdom of } \\
\text { Eswatini (Former } \\
\text { Swaziland) }\end{array}$ & Boyes et al. 1980 \\
\hline 598. & P. spencei Fraser, 1922 & $27(26 A+X)$ & + & India & Dasgupta 1957 \\
\hline 599. & P. whellani Pinhey, 1956 & $25(24 \mathrm{~A}+\mathrm{X})$ & + & $\begin{array}{c}\text { Burkina Faso } \\
\text { (Former Voltiac } \\
\text { Republic) }\end{array}$ & Kiauta and Ochssée 1979 \\
\hline 600. & Pyrrhosoma nymphula (Sutzer, 1776) & $28(26 \mathrm{~A}+\mathrm{XX})^{*}$ & - & Finland & Oksala 1945 \\
\hline \multirow[t]{2}{*}{601.} & \multirow[t]{2}{*}{ Telebasis carmesina Calvert, 1909} & $27(26 \mathrm{~A}+\mathrm{X})$ & - & Surinam & Kiauta 1979a \\
\hline & & $-»-$ & - & Brazil & Ferreira et al. 1979 \\
\hline 602. & Tigriagrion aurantinigrum Calvert, 1909 & $27(26 \mathrm{~A}+\mathrm{X})$ & - & Bolivia & Cumming 1964 \\
\hline
\end{tabular}




\begin{tabular}{|c|c|c|c|c|c|}
\hline \multicolumn{2}{|c|}{ Taxon } & \multirow{2}{*}{$\begin{array}{c}\begin{array}{c}\text { Karyotype } \\
\text { formula } \mathbf{2 n} \delta\end{array} \\
27(26 \mathrm{~A}+\mathrm{X})\end{array}$} & \multirow{2}{*}{\begin{tabular}{|c|}
$\begin{array}{c}\text { m-chromo } \\
\text { somes }\end{array}$ \\
- \\
\end{tabular}} & \multirow{2}{*}{$\begin{array}{c}\text { Country } \\
\text { New Zealand }\end{array}$} & \multirow{2}{*}{$\begin{array}{c}\text { References } \\
\text { Jensen } 1980 \text { as X. zelandica } \\
\text { (McLachlan, 1873) }\end{array}$} \\
\hline 603. & $\begin{array}{l}\text { Xanthocnemis zealandica (McLachlan, } \\
1873 \text { ) }\end{array}$ & & & & \\
\hline 604. & Zoniagrion exclamationis (Selys, 1876) & $27(26 \mathrm{~A}+\mathrm{X})$ & - & USA & Cruden 1968 \\
\hline \multicolumn{6}{|c|}{ Protoneuridae } \\
\hline 605. & Caconeura autumnalis Fraser, 1922 & $25(24 \mathrm{~A}+\mathrm{X})$ & + & India & Tyagi 1978b \\
\hline 606. & Epipleoneura sp. & $27(26 \mathrm{~A}+\mathrm{X})$ & - & Bolivia & Cumming 1964 \\
\hline 607. & Protoneura rubriventris (Selys, 1860) & $27(26 \mathrm{~A}+\mathrm{X})$ & + & Bolivia & $\begin{array}{c}\text { Cumming } 1964 \text { as Neoneura } \\
\text { rubriventris Selys, } 1860\end{array}$ \\
\hline
\end{tabular}

* In the original publication, the female karyotype is given.

** Jensen (1980) considers these data as erroneous (but see section "Concluding remarks and future directions" in the present paper).

*** Karyotype formula is extrapolated based on vague descriptions by Cumming (1964).

Table 2. The diversity of chromosome numbers and sex chromosome mechanisms, and modal karyotypes in 23 families of Odonata: a summary.

\begin{tabular}{|c|c|c|c|c|c|}
\hline \multicolumn{2}{|c|}{$\begin{array}{c}\text { Taxa } \\
\left(\mathrm{N} \text { of species/genera described }{ }^{*}\right)\end{array}$} & $\begin{array}{l}\mathrm{N} \text { of species/ } \\
\text { genera } \\
\text { studied }\end{array}$ & Male karyotypes & $\begin{array}{c}\text { Modal } \\
\text { karyotype }\end{array}$ & $\begin{array}{c}\mathrm{N} \text { of species/genera } \\
\text { with modal karyotype } \\
\text { (occurrence in percent) }\end{array}$ \\
\hline \multicolumn{6}{|l|}{ ANISOZYGOPTERA } \\
\hline Epiophlebioidea & Epiophlebiidae (4/1) & $1 / 1$ & $25, \mathrm{X} 0$ & $24 \mathrm{~A}+\mathrm{X}$ & $1(100) / 1(100)$ \\
\hline \multicolumn{6}{|l|}{ ANISOPTERA } \\
\hline Aeshnoidea & Aeshnidae $(456 / 51)$ & $58 / 18$ & $\begin{array}{c}\text { 13, X0; 14, neo-XY; 15, X0; 16, } \\
\text { neo-XY; 19, X0; 21, X0; 24, } \\
\text { neo-XY; 25, X0; 26, neo-XY; } \\
\text { 27, X0 }\end{array}$ & $26 \mathrm{~A}+\mathrm{X}$ & $44(76) / 14(78)$ \\
\hline Petaluroidea & Petaluridae (10/5) & $4 / 3$ & $17, \mathrm{X} 0 ; 19, \mathrm{X} 0 ; 25, \mathrm{X} 0$ & $16 \mathrm{~A}+\mathrm{X}$ & $3(75) / 2(67)$ \\
\hline Gomphoidea & Gomphidae (980/87) & $66 / 31$ & $\begin{array}{c}\text { 12, neo-neo-XY; 21, X0; 22, } \\
\text { neo-XY; 23, X0; 24, neo-XY; } \\
25, \mathrm{X} 0\end{array}$ & $22 \mathrm{~A}+\mathrm{X}$ & $57(86) / 28(90)$ \\
\hline \multirow[t]{3}{*}{ Libelluloidea } & Macromiidae (125/4) & $6 / 3$ & $25, \mathrm{X} 0$ & $24 \mathrm{~A}+\mathrm{X}$ & $6(100) / 3(100)$ \\
\hline & Corduliidae (154/20) & $23 / 7$ & $\begin{array}{c}\text { 10, neo-XY; } 11, \mathrm{X} 0 ; 13, \mathrm{X} 0 \\
14, \text { neo-XY, } 20, \mathrm{XY} ; 21, \mathrm{X} 0 ; 25 \\
\mathrm{X} 0 ; 26 \text {, neo-XY; } 27, \mathrm{X} 0\end{array}$ & $24 \mathrm{~A}+\mathrm{X}$ & $19(83) / 6(86)$ \\
\hline & Libellulidae (1037/142) & $255 / 59$ & \begin{tabular}{|} 
6, neo-XY; 6 neo-XY; 8, neo- \\
XY; 10, neo-XY; 12, neo-XY; \\
17, X0; 21, X0; 22, neo-XY; 23, \\
X0; 23, X1X2Y; 24, neo-XY; \\
25, X0; 27, X0; 28, neo-XY; 29, \\
X0; 41, X0
\end{tabular} & $24 \mathrm{~A}+\mathrm{X}$ & $227(89) / 57(97)$ \\
\hline \multirow[t]{2}{*}{ Cordulegastroidea } & Cordulegastridae (46/3) & $9 / 3$ & $23, \mathrm{X} 0 ; 25, \mathrm{X} 0$ & $24 \mathrm{~A}+\mathrm{X}$ & $8(89) / 3(100)$ \\
\hline & Chlorogomphidae (47/3) & $1 / 1$ & $25, \mathrm{X} 0$ & $24 \mathrm{~A}+\mathrm{X}$ & $1(100) / 1(100)$ \\
\hline \multicolumn{6}{|l|}{ ZYGOPTERA } \\
\hline \multirow[t]{2}{*}{ Lestoidea } & Lestidae (151/9) & $20 / 5$ & $19, \mathrm{X} 0 ; 21, \mathrm{X} 0 ; 25, \mathrm{X} 0$ & $24 \mathrm{~A}+\mathrm{X}$ & $18(90) / 5(100)$ \\
\hline & Synlestidae (39/9) & $1 / 1$ & $25, \mathrm{X} 0$ & $24 \mathrm{~A}+\mathrm{X}$ & $1(100) / 1(100)$ \\
\hline Platystictoidea & Platystictidae (224/6) & $4 / 3$ & $25, \mathrm{X} 0$ & $24 \mathrm{~A}+\mathrm{X}$ & $4(100) / 3(100)$ \\
\hline \multirow[t]{8}{*}{ Calopterygoidea } & Calopterygidae (185/21) & $20 / 8$ & $23, \mathrm{X} 0 ; 25, \mathrm{X} 0 ; 27, \mathrm{X} 0$ & $24 \mathrm{~A}+\mathrm{X}$ & $20(100) / 8(100)$ \\
\hline & Chlorocyphidae (144/19) & $9 / 6$ & $23, \mathrm{X} 0 ; 25, \mathrm{X} 0$ & $22 \mathrm{~A}+\mathrm{X}$ & $8(89) / 5(84)$ \\
\hline & Polythoridae (59/7) & $2 / 2$ & $23, \mathrm{X} 0$ & $22 \mathrm{~A}+\mathrm{X}$ & $2(100) / 2(100)$ \\
\hline & Euphaeidae (68/12) & $4 / 4$ & $25, \mathrm{X} 0$ & $24 \mathrm{~A}+\mathrm{X}$ & $4(100) / 4(100)$ \\
\hline & Megapodagrionidae (296/42) & $3 / 2$ & $25, \mathrm{X} 0$ & $24 \mathrm{~A}+\mathrm{X}$ & $3(100) / 2(100)$ \\
\hline & Heteragrionidae (57/2) & $2 / 1$ & $25, \mathrm{X} 0$ & $24 \mathrm{~A}+\mathrm{X}$ & $2(100) / 1(100)$ \\
\hline & Philogeniidae (40/2) & $1 / 1$ & $25, \mathrm{X} 0$ & $24 \mathrm{~A}+\mathrm{X}$ & $1(100) / 1(100)$ \\
\hline & Hypolestidae (6/4) & $1 / 1$ & $17, \mathrm{X} 0$ & $16 \mathrm{~A}+\mathrm{X}$ & $1(100) / 1(100)$ \\
\hline \multirow[t]{3}{*}{ Coenagrionoidea } & Platycnemididae (404/40) & $22 / 8$ & $25, \mathrm{X} 0$ & $24 \mathrm{~A}+\mathrm{X}$ & $19(100) / 7(100)$ \\
\hline & Coenagrionidae (1267/114) & $92 / 28$ & $\begin{array}{c}\text { 12, neo-XY; 25, X0; 27, X0; 29, } \\
\text { X0; 30, neo-XY; 37, X0 }\end{array}$ & $26 \mathrm{~A}+\mathrm{X}$ & $81(89) / 26(90)$ \\
\hline & Protoneuridae $(260 / 25)$ & $3 / 3$ & $25, \mathrm{X} 0 ; 27, \mathrm{X} 0$ & $26 \mathrm{~A}+\mathrm{X}$ & $2(70) / 2(70)$ \\
\hline
\end{tabular}

*Taken from Dijkstra et al. 2013 


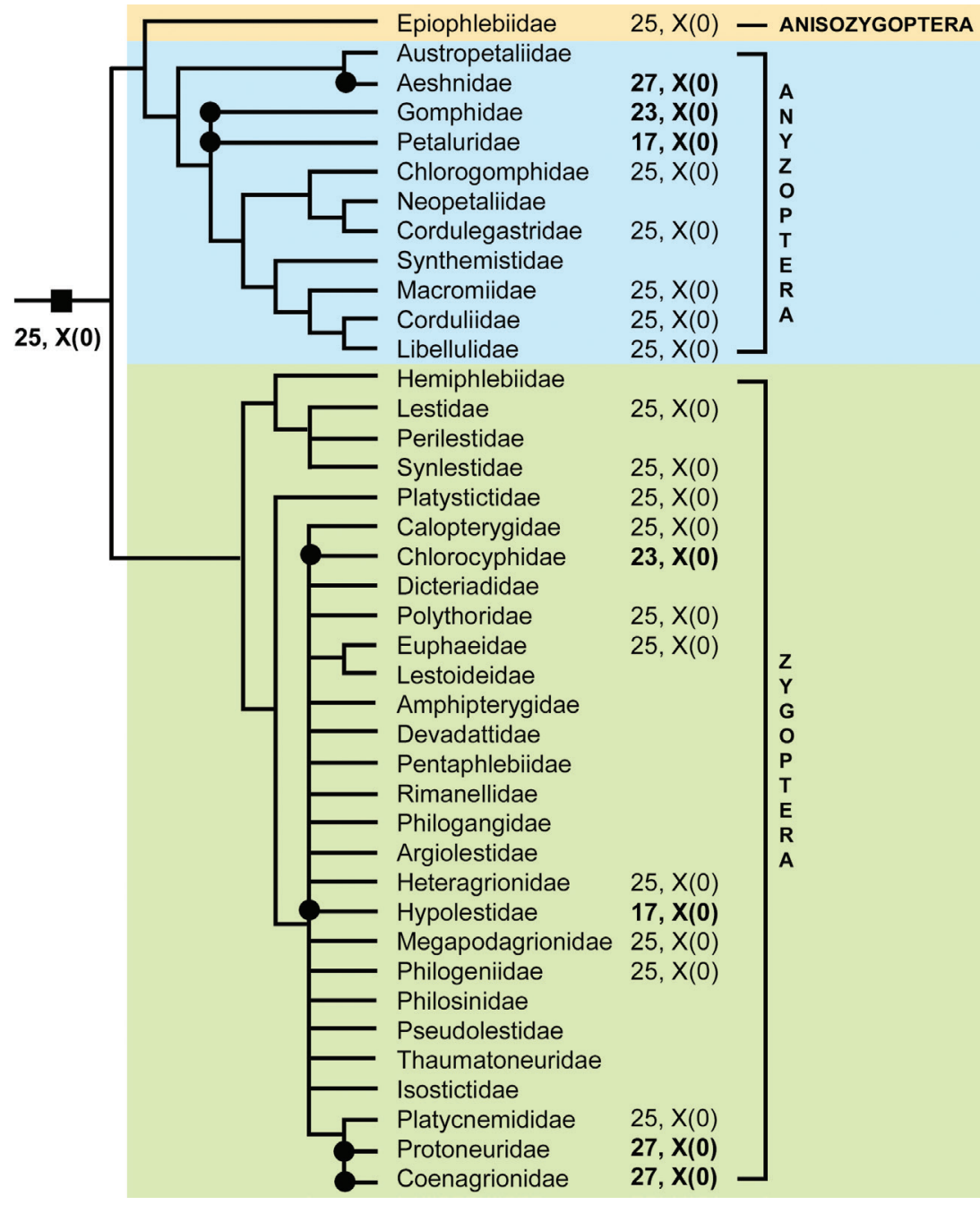

Figure I. Mapping of modal karyotypes onto phylogenetic tree of Odonata families. The phylogenetic tree is taken from Bybee et al. (2016) who synthesized it based on trees from Dijkstra et al. (2014) and Carle et al. (2015). Plesiomorphic karyotype state is indicated by a black solid square (匹), apomorphic karyotype states are indicated by black solid $\operatorname{circles}(\bullet)$.

\section{Concluding remarks and future directions}

In total, karyotypes of 607 species (198 genera, 23 families) of Odonata are studied up to now. Table 1, presented in our work, includes 423 species ( 125 genera, 8 families) of the Anisoptera, 184 species (72 genera, 14 families) of the Zygoptera, and one species of the Anisozygoptera. Thus, the presently available karyotype data cover about $10 \%$ of the world species diversity of the order in general. 
Within Odonata, chromosome numbers in males vary over a relatively wide range, from 2n = 6 in Macrothemis hemichlora and Orthemis levis to 2n = 41 in O. nodiplaga. Both low chromosome number species are suggested to have an evolutionarily secondary neo-XY system (Cumming 1964; Kiauta 1972c) that could have arisen through an $\mathrm{X}$-autosome fusion from an $\mathrm{X}(0)$ system. All three of the above species belong to the largest dragonfly family Libellulidae, in which nearly $89 \%$ of studied species ( 255 in total) have the karyotype $2 n=25(24 \mathrm{~A}+\mathrm{X})$. The last one is the most common in Odonata in general: it occurs in each of the three suborders, Zygoptera, Anisoptera and Anisozygoptera, and in all families with the exception of two damselfly families, the Polythoridae with only two studied species sharing $2 \mathrm{n}=23(22 \mathrm{~A}+\mathrm{X})$ and a monotypic family Hypolestidae with $2 \mathrm{n}=17(16 \mathrm{~A}+\mathrm{X})$ in male Hypolestes clara. Besides Libellulidae, the karyotype $2 \mathrm{n}=25(24 \mathrm{~A}+\mathrm{X})$ is currently the presumed modal one in 14 other families, such being the case at least in six better covered (at species and/ or generic level) families, i.e. the dragonfly families Corduliidae, Cordulegastridae, and Macromiidae, and the damselfly families Lestidae, Calopterygidae, and Platycnemididae (Table 2, Fig. 1). This chromosome set is suggested to be an ancestral one for the order Odonata in general (Oguma 1930; Kuznetsova et al. 2020b) although this suggestion remains questionable at this stage.

Chromosomal rearrangements, among which fission and fusions apparently predominated (Kiauta 1969c, 1972c), led to the appearance of divergent karyotypes in the evolution of Odonata. As a result, in many dragonfly and damselfly families, other karyotypes, when occurring, are of secondary origin as indicated by either a diverged number of autosomes or a secondary sex chromosome system of an XY-type or both (e.g. Cumming 1964; Kiauta 1969a, c; Agopian and Mola 1984, 1988; Mola et al. 1999; Perepelov and Bugrov 2002). Some interesting examples of this kind can be found in the family Libellulidae, in which $2 \mathrm{n}=25(24 \mathrm{~A}+\mathrm{X})$ is most likely an evolutionarily initial karyotype (e.g. Agopian and Mola 1988). These examples are as follows (see Table 1): Orthemis nodiplaga and O. ambinigra with $2 \mathrm{n}=41(40 \mathrm{~A}+\mathrm{X})$ and $2 \mathrm{n}=12(10 \mathrm{~A}+$ neo-XY $)$, respectively; Erythrodiplax media and E. minuscula, both with $2 \mathrm{n}=22(20 \mathrm{~A}+\mathrm{neo}-\mathrm{XY})$; Micrathyria longifasciata and $M$. ungulata with $2 \mathrm{n}=24(22 \mathrm{~A}+\mathrm{neo}-\mathrm{XY})$ and $2 \mathrm{n}=23\left(20 \mathrm{~A}+\mathrm{X}_{1} \mathrm{X}_{2} \mathrm{Y}\right)$, respectively. In some families, any of these presumably derived karyotypes not only occurs but also prevails and may be considered modal (see Table 2 and Fig. 1). Within Anisoptera, such families are Aeshnidae $(2 \mathrm{n}=26 \mathrm{~A}+\mathrm{X})$ and Gomphidae $(2 \mathrm{n}=22 \mathrm{~A}+\mathrm{X})$, whereas within Zygoptera, these are Chlorocyphidae $(2 \mathrm{n}=22 \mathrm{~A}+\mathrm{X})$ and Coenagrionidae $(2 \mathrm{n}=26 \mathrm{~A}+\mathrm{X})$. Thus, Odonata, despite the fact that they have holokinetic chromosomes (Nokkala et al. 2002), demonstrate rather high karyotypic stability, with most species showing $2 \mathrm{n}=25$ (found in $60 \%$ of studied species), $2 \mathrm{n}=27(21 \%)$ and $2 \mathrm{n}=23(13 \%)$ which may point to some selective constraints acting to stabilize chromosome number in their evolution (Kuznetsova et al. 2020b).

There are the species for which different authors give various karyotypes that are sometimes difficult to interpret (see Table 1). In some cases, this might be due to 
misidentifications of a particular species or an error in determining the karyotype. For example, Wolfe (1953) reported $2 \mathrm{n}=17(16 \mathrm{~A}+\mathrm{X})$ for males of Uropetala carovei (Petaluridae, Anisoptera) from New Zealand. However, according to later studies of this species in the same locality (Jensen and Mahanty 1978; Jensen 1980), it has 2n $=25(24 \mathrm{~A}+\mathrm{X})$, and Jensen (1980) therefore considers the Wolfe data as erroneous. We cannot exclude, however, that the above authors studied different $U$. carovei subspecies, $U$. c. carovei White, 1846 and U. c. chiltoni Tillyard, 1921, that may indeed have different karyotypes. In other cases, the chromosome number difference between geographic populations might be indicative of the inter-population variation within the bounds of one taxonomic species or even the existence of a species complex with several morphologically cryptic species. For example, 4 of the 17 studied species of the dragonfly genus Aeshna Fabricius, 1775 were reported to have different karyotypes in different populations. These are: Aeshna grandis $-2 \mathrm{n}=26 \mathrm{~A}+\mathrm{X}$ (former USSR), $2 \mathrm{n}=24 \mathrm{~A}+\mathrm{X}$ (former USSR, Finland), and $2 \mathrm{n}=24 \mathrm{~A}+$ neo-XY (Netherlands, Finland); $A$. isoceles $-2 \mathrm{n}=26 \mathrm{~A}+\mathrm{X}(\mathrm{USA})$ and $2 \mathrm{n}=24 \mathrm{~A}+\mathrm{X}$ (Russia); . juncea $-2 \mathrm{n}=26 \mathrm{~A}+\mathrm{X}$ (Italy) and $2 \mathrm{n}=24 \mathrm{~A}+$ neo-XY (Finland, former USSR, Italy); $A$. mixta $-2 \mathrm{n}=26 \mathrm{~A}+\mathrm{X}$ (Netherlands) and $2 \mathrm{n}=24 \mathrm{~A}+\mathrm{X}$ (India) (Table 1). In all such cases, special studies involving a combined analysis of karyotypes, morphology, distribution patterns and molecular markers are needed.

Approximately $80 \%$ of Odonata species have a pair of very small chromosomes, i.e. microchromosomes or m-chromosomes (Mola 2007, Table 1). A number of speculations have been forwarded to explain the origin of these chromosomes in Odonata. Kiauta (1968e) suggested m-chromosomes to be fragments of "normal" chromosomes, whereas Oguma (1930) considered them the remnants of an autosome pair in the process of its elimination by progressive loss of chromatin. The size of the smaller chromosome pair was shown to be variable within different species (Kiauta 1968e; see Mola 2007 for other references) which is consistent with both hypotheses. Closely related species and different populations of the same species often differ from each other in the presence/absence of m-chromosomes (Table 1). This is most likely due to the lack of clear criteria for the identification of a small chromosome pair as m-chromosomes in a particular karyotype (Mola 2007; Kuznetsova et al. 2020b).

Most cytogenetic studies of Odonata have been made only to determine the chromosome number and sex chromosome mechanism for which the routine staining was used. Although a considerable amount of such data was obtained (Table 1,2), standard karyotypes of many Odonata taxa remain totally unknown (Fig. 1). Lack of data on more "primitive" families of Zygoptera (e.g. Hemiphlebiidae) and Anisoptera (e.g. Austropetaliidae and Neopetaliidae) makes difficult understanding karyotype evolution of the order in general.

During the last decades, karyotypes of a few dozen Odonata species were studied using various techniques of differential staining of chromosomes such as C-banding, AgNOR-staining and DNA specific fluorochrome banding visualiszing constitutive heterochromatin, nucleolus organizing regions (NORs) and AT- and GC-rich chromosome segments, respectively. Such data can be found in the following publica- 
tions: Thomas and Prasad (1986), Prasad and Thomas (1992), Perepelov et al. (1998), Perepelov and Bugrov (2001a, b, 2002), Grozeva and Marinov (2007), De Gennaro et al. (2008), Walia et al. (2011, 2018), Walia and Chahal $(2014,2018)$, Walia and Devi (2018), Walia and Katnoria (2018), Walia and Devi (2020a, b). Unfortunately, these data alone did not shed much light on the karyotypic evolution of Odonata.

Although the classical cytological techniques remain necessary starting points for cytogenetic studies of Odonata to get an overview of their genomes, the future of Odonata cytogenetics must be coupled with the application of new cytogenetic molecular techniques that enable the localization of specific DNA sequences in chromosomes and the identification of individual chromosomes in karyotypes. In the article by Frydrychová et al. (2004) and, on a larger scale, in two of our recent publications (Kuznetsova et al. 2018, 2020b), the fluorescence in situ hybridization (FISH) technique was used for the first time for analyzing Odonata karyotypes. Several species belonging to the Anisoptera (from the families Aeshnidae, Libellulidae, and Corduliidae) and the Zygoptera (from the families Coenagrionidae and Calopterygidae) were studied regarding the occurrence of the TTAGG telomeric repeats and the distribution of the $18 S r R N A$ genes in their karyotypes. The TTAGG repeats proved to be the canonical motif of telomeres in the class Insecta in general, which, however, was repeatedly lost in the evolution of different phylogenetic lineages (Kuznetsova et al. 2020a). It was shown in the listed Odonata publications that the (TTAGG) motif does not occur in all but one (Sympetrum vulgatum) species, and the $18 \mathrm{~S}$ is located on one of the largest pairs of autosomes in all studied dragonfly species but on m-chromosomes in all studied damselfly species (Kuznetsova et al. 2020b).

The results obtained showed great promise of the combined use of FISH and classical and banding cytogenetics in order to identify new chromosomal markers, reveal differences between species, particularly when they share the same or very close karyotypes, and speculate about the mechanisms involved in the karyotype evolution of Odonata (Kuznetsova et al. 2020b). Another promising line of future research could be to test hypotheses (Mola and Papeschi 1994; Ardila-Garcia and Gregory 2009) about whether there is a relationship between karyotype evolution and genome size diversity in the Odonata or there is no such relationship.

\section{Acknowledgements}

The present study was performed within the research project no. AAAAA19-119020790106-0. The authors deeply grateful to the late Evgeny A. Perepelov whose thesis "Karyotype evolution of Odonata (Insecta) of Northern Palearctics" (2003; Novosibirsk State University, Novosibirsk, Russia) served as a starting point for preparing Table 1 of our paper. We thank two reviewers, Dr. M. Marinov and Dr. S. Grozeva, for their useful remarks to a draft of this MS. Special thanks to Dr. M. Marinov for his nomenclatural and taxonomic corrections and updates. 


\section{References}

Agopian SS, Mola LM (1984) An exceptionally high chromosome number in Orthemis nodiplaga Kersch (Anisoptera, Libellulidae). Notulae Odonatologicae 2(3): 45.

Agopian SS, Mola LM (1988) Intra and interspecific karyotype variability in five species of Libellulidae (Odonata, Anisoptera). Caryologia 41(1): 69-78. https://doi.org/10.1080/00 087114.1988.10797849

Ardila-Garcia AM, Gregory TR (2009) An exploration of genome size diversity in dragonflies anddamselflies (Insecta: Odonata). Journal of Zoology 278: 163-173. https://doi. org/10.1111/j.1469-7998.2009.00557.x

Asana JJ, Makino S (1935) A comparative study of the chromosomes in the Indian dragonflies. Journal of the Faculty of Science, Hokkaido University Series 6, Zoology 4(2): 67-86.

Boyes JW, van Brink JM, Kiauta B (1980) Sixteen dragonfly karyotypes from the republic of South Africa and Swaziland, with evidence on the possible hybrid nature of Orthetrum julia falsum Longfeild (Anisoptera: Libellulidae). Odonatologica 9: 131-145.

Bybee S, Córdoba-Aguilar A, Duryea MC, Futahashi R, Hansson B, Lorenzo-Carballa MO, Schilder R, Stoks R, Suvorov A, Svensson EI, Swaegers J, Takahashi J, Watts PC, Wellenreuther M (2016) Odonata (dragonflies and damselflies) as a bridge between ecology and evolutionary genomics. Frontiers in Zoology 13: e46. https://doi.org/10.1186/s12983-016-0176-7

Capitulo RA, Mola LM, Agopian SS (1991) Species catalogue and chromosomal data of Odonata from Argentina. Revista de la Sociedad Entomológica Argentina 49(1-4): 59-72.

Carle FL, Kjer KM, May ML (2015) A molecular phylogeny and classification of Anisoptera (Odonata). Arthropod Systematics and Phylogeny 73(2): 281-301. https://entomology. rutgers.edu/news/docs/Carle-2015-Anisoptera-Phylogeny-Classification.pdf

Carnoy BJ (1885) La cytodierese chez les arthropodes. IV. Pseudo-Nevropteres. Cellule 1: 279-282. Chatterjee K, Kiauta B (1973) Male germ cell chromosomes of two Calopterygoidea from the Darjeeling Himalaya (Zygoptera: Chlorocyphidae, Euphaeidae). Odonatologica 2(2): 105-108.

Cruden RW (1968) Chromosome numbers of some North American dragonflies (Odonata). Canadian Journal of Genetics and Cytology 10: 200-214. https://doi.org/10.1139/g68-029

Cumming RB (1964) Cytogenetic studies in the order Odonata. PhD thesis, University of Texas, Austin, 93 pp.

Das C (1956) Studies on the association between non-homologous chromosomes during meiosis in four species of the Indian dragonflies (Odonata). Journal of the Zoological Society of India 8(2): 119-132.

Dasgupta J (1957) Cytological studies of some Indian dragonflies. II: A study of the chromosomes during meiosis in thirty species of Indian Odonata (Insecta). Proceedings of the Zoological Society of Calcutta 10: 1-65.

De Gennaro D (2004) Análisis meiótic y caracterización de la heterocromatina en species argentinas de Anizoptera (Odonata). Tesis de Licenciatura. Facultad de Ciencias Exactas y Naturales de la Universidad de Buenos Aires. Buenos Aires, 66 pp.

De Gennaro D, Rebagliati PJ, Mola LM (2008) Fluorescent banding and meiotic behaviour in Erythrodiplax nigricans (Libellulidae) and Coryphaeschna perrensi (Aeschnidae) (Anisoptera, Odonata). Caryologia 61: 60-67. https://doi.org/10.1080/00087114.2008.10589610 
Dijkstra K-DB, Bechly G, Bybee SM, Dow RA, Dumont HJ, Fleck G, Garrison RW, Hämäläinen M, Kalkman VJ, Karube H, May ML, Orr AG, Paulson DR, Rehn AC, Theischinger G, Trueman JWH, van Tol J, Ellenrieder N, Ware J (2013) The classification and diversity of dragonflies and damselflies (Odonata). In: Zhang ZQ (Ed.) Animal Biodiversity: An Outline of Higher-level Classification and Survey of Taxonomic Richness (Addenda 2013). Zootaxa 3703: 36-45. https://doi.org/10.11646/zootaxa.3703.1.9

Dijkstra K-DB, Kalkman VJ, Dow RA, Stokvis FR, van Tol J (2014) Redefining the damselfly families: a comprehensive molecular phylogeny of Zygoptera (Odonata). Systematic Entomology 39: 68-96. https://doi.org/10.1111/syen.12035

Ferreira A, Kiauta B, Zaha A (1979) Male germ cell chromosomes of thirty-two Brazilian dragonflies. Odonatologica 8: 5-22.

Francovič M, Jurečic R (1986) Prilog citogenetickim i citotaksonomskim istrazivanjima vrste Libellula depressa L. (Odonata, Libellulidae). Plenarni Referati VII Kongres Biologa Jugoslavije, Budva, 341 pp.

Francovič M, Jurečic R (1989) Comparative cytogenetic analysis of karyotype morphology and organization in males of species Libellula depressa L. and L. fulva Müll. (Insecta: Odonata). Periodicum Biologorum 91(1): 32-33.

Frydrychová R, Grossmann P, Trubač P, Vítková M, Marec F (2004) Phylogenetic distribution of TTAGG telomeric repeats in insects. Genome 47: 163-178. https://doi.org/10.1139/g03-100 Fuchsówna J, Sawczyńska J (1928) Zachowanie sie heterochromosomóv podczam spermatogenezy u wažek (Odonata). Cz. I. Aeschna grandis L. Libellula quadrimaculata L. Archiwum Towarzystwa naukowego we Lwowie (III) 4(9): 177-197. [In Polish]

Goni B, de Abenante YP (1982) Cytological notes on five dragonfly species from Uruguay. Odonatologica 11(4): 323-329.

Grimaldi D, Engel MS (2005) Evolution of the Insects. Cambridge University Press, Cambridge, $755 \mathrm{pp}$.

Grozeva SM, Marinov MG (2007) Cytogenetic study of Somatochlora borisi Marinov, 2001 (Odonata: Corduliidae), and three relative species. Acta Zoologica Bulgarica 59(1): 53-58. Handa SM, Batra HN (1980) Cytology of ten species of dragonflies (Anisoptera: Odonata). Proceedings of the $67^{\text {th }}$ Indian Science Congress, Part III, Calcutta, 103 pp.

Handa SM, Kochhar N (1985) Chromosomal architecture in two species of damselflies from Chandigarh and its surrounding areas. National Seminar on Current Trends in Chromosome Dynamics, Chandigarh, $34 \mathrm{pp}$.

Handa SM, Mittal OP, Batra HN (1984) Chromosomes in ten species of dragonflies (Anisoptera: Odonata). Research Bulletin of the Panjab University (Science) 35: 65-75.

Higashi K, Kayano H (1993) The distribution of distinct karyomorphs of Crocothemis servilia Drury (Anisoptera, Libellulidae) in Kyushu and the south-western islands of Japan. Japanese Journal of Entomology 61: 1-10.

Higashi K, Lee CE, Kayano H, Kayano A (2001) Korea strait delimiting distribution of distinct karyomorphs of Crocothemis servilia (Drury) (Anisoptera: Libellulidae). Odonatologica 30(3): 265-270.

Hirai H (1956) Chromosomes of six species of dragonflies. Zoological Magazine, Tokyo 65: 198-202. 
Hogben L (1921) Studies on synapsis, III. The nuclear organisation of the germ cells in Libellula depressa. Philosophical Transactions of the Royal Society of London Series B 92: 60-80. https://doi.org/10.1098/rspb.1921.0006

Hung ACF (1971) Cytological studies of five dragonflies (Odonata: Anisoptera). Entomological News 82: 103-106.

Jensen AL (1980) The karyotypes of five species of Odonata endemic to New Zealand. Odonatologica 9: 29-33.

Jensen AL, Mahanty HK (1978) A preliminary note on the chromosome number of Uropetala carovei (White) (Anisoptera: Petaluridae). Odonatologica 7: 385-386.

Kalkman VJ, Clausnitzer V, Dijkstra K-DB, Orr AG, Paulson DR, van Tol J (2008) Global diversity of dragonflies (Odonata) in freshwater. Hydrobiologia 595: 351-363. https://doi. org/10.1007/s10750-007-9029-x

Katatani N (1987) On the chromosomes of dragonflies, 1. Synopsis on the studies in some Japanese dragonflies. Aeschna 20: 21-31.

Kiauta B (1965) The chromosome behaviour in spermatogenetic meiosis of Anax imperator Leach (Odonata: Aeshnidae). Tombo 7(3-4): 18-21.

Kiauta B (1966) The chromosome behaviour in spermatogenetic meiosis of the dragonfly Sympetrum striolatum (Charp.) (Odonata: Libellulidae) from Luxembourg. Bulletin de la Société des Naturalistes Luxembourgeois 69: 54-60.

Kiauta B (1967a) Evolution of the chromosome complement in Odonata. Genen en Phaenen 11(4): 56-61.

Kiauta B (1967b) Abstract. Evolution of the chromosome complement in Odonata. Genetica 38(3): 403-404. https://doi.org/10.1007/BF01507474

Kiauta B (1967c) A new hypothesis on the evolution of the chromosome complement in Odonata. Tombo 10(1-4): 29-33.

Kiauta B (1967d) Considerations on the evolution of the chromosome complement in Odonata. Genetica 38(4): 430-446. https://doi.org/10.1007/BF01507474

Kiauta B (1967e) Meiotic chromosome behaviour in the male damselfly, Calopteryx virgo (Linnaeus), with a discussion on the value of chromosome numbers and karyotype morphology in odonate systematics. Deutsche Entomologische Zeitschrift 14(3-4): 339-348. https://doi.org/10.1002/mmnd.19670140312

Kiauta B (1968a) Evolution of the chromosome complement in Odonata. Entomologische Berichten, Amsterdam 28(5): 97-100.

Kiauta B (1968b) Morphology and kinetic behaviour of the odonate sex chromosomes, with a review of the distribution of sex determining mechanisms in the order. Genen en Phaenen 12(1): 21-24.

Kiauta B (1968c) The chromosome numbers of eight Old World dragonflies (Odonata). Chromosome Information Service, Tokyo 9: 3-4.

Kiauta B (1968d) The chromosomes of the male dragonfly Cordulegaster boltoni (Donovan, 1807) (Odonata: Cordulegasteridae). Biološki Vestnik: glasilo slovenskih biologov 16: 87-94.

Kiauta B (1968e) Variation in size of the m-chromosome of the dragonfly, Calopteryx virgo (L.), and its significance for the chorogeography and taxonomy of the Calopteryx virgo superspecies. Genen en Phaenen 12(1): 11-16. 
Kiauta B (1968f) Variation in size of the dragonfly m-chromosome, with considerations on its significance for the chorogeography and taxonomy of the order Odonata, and notes on the validity of the rule of Reinig. Genetica 39(1): 64-74. https://doi.org/10.1007/BF02324456

Kiauta B (1969a) Sex chromosomes and sex determining mechanisms in Odonata, with a review of the cytological conditions in the family Gomphidae, and reference to the karyotypic evolution in the order. Genetica 40(2): 127-157. https://doi.org/10.1007/BF01787346

Kiauta B (1969b) The chromosomes of eight dragonfly species from continental Africa and Madagascar (Odonata). Arnoldia (Rhodesia) 4(15): 1-8.

Kiauta B (1969c) Autosomal fragmentations and fusions in Odonata and their evolutionary implications. Genetica 40(2): 158-180. https://doi.org/10.1007/BF01787347

Kiauta B (1969d) The chromosomes of the Hawaiian endemic dragonflies, Megalagrion oahuense (Blackburn) (Coenagrionidae: Pseudagrioninae) and Nesogonia blackburni (McLachlan) (Libellulidae: Sympetrinae), with a note on the cytotaxonomic affinities between the genera Nesogonia Kirby and Sympetrum Newman (order Odonata). Proceedings of the Hawaiian Entomological Society 20(2): 429-433.

Kiauta B (1970a) The chromosomes of four Neotropical dragonflies from Mexico. Chromosome Information Service, Tokyo 11: 8-9.

Kiauta B (1970b) The karyotype of the damselfly, Epallage fatime (Charpentier, 1840) (Odonata, Zygoptera: Epallagidae), with a note on the cytotaxonomic affinities in the superfamily Calopterygoidea. Genetica 41: 390-397. https://doi.org/10.1007/BF00958931

Kiauta B (1971a) Studies on the germ cell chromosome cytology of some cytotaxonomically interesting or hitherto not studied Odonata from the autonomous region Friuli-Venezia Giulia (northern Italy). Atti del Museo civico di Storia naturale di Trieste 27: 65-127.

Kiauta B (1971b) An unusual case of precocious segregation and chromosome fragmentation in the primary spermatocytes of the damselfly, Calopteryx virgo meridionalis (Selys, 1873), as evidence for a possible hybrid character of some populations of the Calopteryx-virgocomplex (Odonata, Zygoptera: Calopterygidae). Genen en Phaenen 14(2): 32-40.

Kiauta B (1971c) Cytotaxonomic peculiarities in the neotropical odonate genera Leptagrion Selys, Orthemis Hagen and Macrothemis Hagen. Abstracts of papers read at the $1^{\text {st }}$ European Symposium on Odonatology, Gent, 27-28.

Kiauta B (1972a) Notes on new or little known dragonfly karyotypes, 2. Male germ cell chromosomes of four East Mediterranean species: Lestes barbarus (Fabricius), Calopteryx splendens amasina Bartenev (Zygoptera: Lestidae, Calopterygidae), Caliaeschna microstigma (Schneider) and Orthetrum taeniolatum (Schneider) (Anisoptera: Aeshnidae, Libellulidae). Genen en Phaenen 15: 95-98.

Kiauta B (1972b) Notes on new or little known dragonfly karyotypes, 1. The germ cell chromosomes of three Latin American species: Argia funebris (Hagen), Megapodagrion contortum (Selys) (Zygoptera: Coenagrionidae, Megapodagrionidae) and Castoraeschna castor (Brauer) (Anisoptera: Aeshnidae). Genen en Phaenen 15: 23-26.

Kiauta B (1972c) Synopsis on the main cytotaxonomic data in the order Odonata. Odonatologica 1(2): 73-102.

Kiauta B (1972d) The karyotype of the damselfly, Leptagrion macrurum (Burmeister, 1839), and its possible origin, with a note on the cytotaxonomic affinities of the genus (Zygoptera: Coenagrionidae). Odonatologica 1(1): 31-35. 
Kiauta B (1973a) Notes on new or little known dragonfly karyotypes. III. Spermatocyte chromosomes of four Nearctic anisopterans: Aeshna californica Calvert (Aeshnidae), Cordulia shurtleffi Scudder (Corduliidae), Sympetrum internum Montgomery, and S. madidum (Hagen) (Libellulidae). Genen en Phaenen 16(1): 7-12.

Kiauta B (1973b) Notes on new or little known dragonfly karyotypes. IV. Spermatocyte chromosomes of Calopteryx splendens splendens Harris (Zygoptera: Calopterygidae), Gomphus pulchellus Selys, Libellula depressa Linnaeus (Anisoptera: Gomphidae, Libellulidae) from northern France. Genen en Phaenen 16(2): 55-60.

Kiauta B (1974) Introduction to insect cytotaxonomy. Lectures delivered at the Tribhuvan University, Kathmandu, Vol. 1. Nepal Research Center, Kathmandu, 81 pp.

Kiauta B (1975) Cytotaxonomy of dragonflies, with special reference to the Nepalese fauna. Lectures delivered at the Tribhuvan University, Kathmandu, Vol. 2. Nepal Research Center, Kathmandu, 78 pp.

Kiauta B (1977) Notes on new or little known dragonfly karyotypes.V. The male germ cell chromosomes of Macromia moorei Selys from Nepal (Anisoptera: Corduliidae, Epophthalmiinae). Genen en Phaenen 19: 49-51.

Kiauta B (1978) Two cytotaxonomically interesting cases of irreversible autosome fusion in dragonflies Agria modesta (Hagen) (Zygoptera: Coenagrionidae) and Anaciaeschna isosceles (Müller) (Anizoptera: Aeshnidae). Notulae Odonatologicae 1(1): 7-9.

Kiauta B (1979a) The karyotypes of some Anisoptera from Surinam. Odonatologica 2: 267-283. Kiauta B (1979b) The karyotype of Ischnura pumilio (Charp.) (Zygoptera: Coenagrionidae). Notulae Odonatologicae 1(3): 47-48.

Kiauta B (1983) The status of the Japanese Crocothemis servilia (Drury) as revealed by karyotypic morphology (Anisoptera: Libellulidae). Odonatologica 12: 381-388.

Kiauta B, Boyes JW (1972) Cytology of ten South American Libellulidae, with cytophylogenetic consideration of the genera Orthemis Hagen and Erythrodipax Brauer (Odonata, Anisoptera). Genetica 43(3): 407-421. https://doi.org/10.1007/BF00156136

Kiauta B, Brink JM (1975) Cytotaxonomic notes on the Sympetrum pedemontanum complex (Anisoptera: Libellulidae). Odonatologica 4(4): 249-254.

Kiauta B, Brink JM (1978) Male chromosome complements of some Florida dragonflies, United States. Odonatologica 7(1): 155-25.

Kiauta B, Kiauta MAJE (1976) The chromosomes of some dragonflies from the Langtang Valley, Central Nepal. Odonatologica 5(4): 347-354.

Kiauta B, Kiauta MAJE (1979) The karyotype of Libellula fulva Müll, from Switzerland (Anisoptera: Libellulidae). Notulae Odonatologicae 1(4): 73-74.

Kiauta B, Kiauta MAJE (1980a) The karyotypes of Aeshna subarctica elisabethae Djak. and Somatochlora alpestris (Sel.) from Switzerland (Anisoptera, Aeshnidae, Corduliidae). Notulae Odonatologicae 1(6): 104-105.

Kiauta B, Kiauta MAJE (1980b) On a small collection of dragonfly karyotypes from the Philippines. Odonatologica 9(3): 237-245.

Kiauta B, Kiauta MAJE (1980c) Introduction to the cytotaxonomy of the odonate genus Agria Rambur (Zygoptera: Coenagrionidae). Odonatologica 9(1): 35-56.

Kiauta B, Kiauta MAJE (1982) The chromosome numbers of sixteen dragonfly species from the Arun Valley, Eastern Nepal. Notulae Odonatologicae 9(1): 143-146. 
Kiauta B, Kiauta MAJE (1983) The chromosome numbers of some Odonata from Thailand. Notulae Odonatologicae 2(2): 17-32.

Kiauta B, Kiauta MAJE (1991) Biogeographic considerations on Coenagrion hylas freyi (Bilek, 1954), based mainly on the karyotype features of a population from North Tyrol, Austria (Zygoptera: Coenagrionidae). Odonatologica 20(4): 417-431.

Kiauta B, Kiauta MAJE (1995) The karyotypes of Somatochlora meridionalis Nielsen from Slovenia and S. metallica (Vander L.) from Switzerland, with a tentative note on the origin of Central European S. metallica (Odonata: Corduliidae). Opuscula zoologica fluminensia 137: 1-5. Kiauta B, Kiauta-Brink MAJE (1975) Chromosomes of the dragonfly, Sympecma annulata braueri (Yakobson \& Bianki, 1905) from the Netherlands, with a note on the classification of the family Lestidae (Odonata, Zygoptera). Genen en Phaenen 18(2-3): 39-48.

Kiauta B, Ochssée BV (1979) Some dragonfly karyotypes from the Voltiac Republic (Haute Volta), West Africa. Odonatologica 8: 47-54.

Kichijo H (1939) Chromosomes of Tachopteryx pryeri and Gomphus hakiensis (Odonata, Aeshnidae). Japanese Journal of Genetics 15: 287-289. https://doi.org/10.1266/jjg.15.287

Kichijo H (1941) Chromosomes of seven species of insects belonging to the order of dragonflies, suborder of damselfies. Nagasaki Medical Journal 19(10): 2033-2041. [In Japanese]

Kichijo H (1942a) Insect chromosomes. IV. Order of dragonflies, Pt. 2. Nagasaki Medical Journal 20(10): 1639-1648. [In Japanese]

Kichijo H (1942b) Insect chromosomes. III. Order of dragonflies, Pt. 1. Nagasaki Medical Journal 20(7): 1084-1092. [In Japanese]

Kichijo H (1942c) Chromosomes of Sympetrum eroticum eroticum (Odonata). Japanese Journal of Genetics 18: 195-196. https://doi.org/10.1266/jjg.18.195

Kichijo H (1942d) A comparative study of seven species of Zygoptera from Japan. Acta medica Nagasakiensi 3(2): 95-97.

Kichijo H (1942e) On the chromosomes of some species of the zygopterous dragonflies (Odonata, Zygoptera). Japanese Journal of Genetics 18: 273-276. https://doi.org/10.1266/jjg.18.273

Kumari U, Gautam DC (2017) Karyotypic studies on two species of Orthetrum (Anisoptera: Odonata) from Himachal Pradesh. The Journal of Cytology and Genetics 18: 1-7.

Kuznetsova V, Grozeva S, Gokhman V (2020a) Telomere structure in insects: A review. Journal of Zoological Systematics and Evolutionary Research 58: 127-158. https://doi. org/10.1111/jzs.12332

Kuznetsova VG, Maryańska-Nadachowska A, Shapoval NA, Anokhin BA, Shapoval AP (2018) Cytogenetic characterization of eight Odonata species originating from the Curonian Spit (the Baltic Sea, Russia) using C-banding and FISH with $18 S$ rDNA and telomeric (TTAGG)n n probes. Cytogenetic and Genome Research 153: 147-157. https://doi. org/10.1159/000486088

Kuznetsova VG, Maryańska-Nadachowska A, Anokhin BA, Shapoval NA, Shapoval AP (2020b) Chromosomal analysis of eight species of dragonflies (Anisoptera) and damselflies (Zygoptera) using conventional cytogenetics and FISH: insights into the karyotype evolution of the ancient insect order Odonata. Journal of Zoological Systematics and Evolutionary Research 58, 00: 1-13. https://doi.org/10.1111/jzs.12429 [in press]

Lefevre G, McGill C (1908) The chromosomes of Anasa tristis and Anax junius. The American Journal of Anatomy 7(4): 469-487. https://doi.org/10.1002/aja.1000070404 
Makalowskaja WN (1940) Comparative karyological studies of dragonflies (Odonata). Archives russes d'Anatomie, d'Histologie et d'Embryologie 25: 24-39.

Makino S (1935) A comparative study of the chromosomes in the Indian dragonflies. Japanese Journal of Genetics 11: 234-235. https://doi.org/10.1266/jjg.11.234

McGill C (1904) The spermatogenesis of Anax junius. University of Missouri Studies 2: 236-250.

McGill C (1907) The behavior of the nucleoli during oogenesis of the dragonfly with special reference to synapsis. Zoologische Jahrbücher. Abteilung für Anatomie und Ontogenie der Tiere 23: 207-230.

Mola LM (1995) Post-reductional meiosis in Aeshna (Aeshnidae, Odonata). Hereditas 122: 47-55. https://doi.org/10.1111/j.1601-5223.1995.00047.x

Mola LM (1996) Meiotic studies in nine species of Erythrodiplax (Libellulidae, Odonata). NeoXY sex chromosome system in Erythrodiplax media. Cytologia 61: 349-357. https://doi. org/10.1508/cytologia.61.349

Mola LM (2007) Cytogenetics of American Odonata. In: Tyagi BK (Ed.) Odonata: Biology of Dragonflies. Scientific Publishers, India, 153-173.

Mola LM, Agopian SS (1985) Observations on the chromosomes of four South American Libellulidae (Anisoptera). Odonatologica 14(2): 115-125.

Mola LM, Papeschi AG (1994) Karyotype evolution in Aeshna (Aeshnidae: Odonata). Hereditas 121: 185-189. https://doi.org/10.1111/j.1601-5223.1994.00185.x

Mola LM, Papeschi AG, Carrillo ET (1999) Cytogenetics of seven species of dragonflies. Hereditas 131: 147-153. https://doi.org/10.1111/j.1601-5223.1999.00147.x

Nokkala S, Laukkanen A, Nokkala C (2002) Mitotic and meiotic chromosomes in Somatochlora metallica (Corduliidae, Odonata). The absence of localized centromeres and inverted meiosis. Hereditas 136: 7-12. https://doi.org/10.1034/j.1601-5223.2002.1360102.x

Oguma K (1915) A study of the chromosomes of dragonflies. Zoological Magazine 27: 241250. [In Japanese]

Oguma K (1917) Entomology and cytology. In: Nagano K (Ed.) A Collection of Essays for Mr. Yasushi Nawa, Written in Commemoration of His Sixtieth Birthday, October 8, 1917. Gifu, 105-114.

Oguma K (1930) A comparative study of the spermatocyte chromosome in allied species of the dragonfly. Journal of Faculty of Sciences, Hokkaido University VI: 1-32.

Oguma K (1942) Observationes de formis compositionibusque chromosomatum et dispositionibus eorum in tempore divisionis atque propositio aliquorum novorum terminorum. Japanese Journal of Genetics 18: 205-216. https://doi.org/10.1266/jig.18.205

Oguma K (1951) The chromosomes of Epiophlebia superstes Selys (dragonfly). Iden-no-SogoKenkyu 2: 23-26.

Oguma K, Asana JJ (1932) Additional data to our knowledge on the dragonfly chromosome with a note on the occurrence of X-Ychromosome in the ant-lion (Neuroptera). Journal of Faculty of Sciences, Hokkaido University 1(4): 133-142.

Oksala T (1939a) Über Tetraploidie der Binde- und Fettgewebe bei den Odonaten. Hereditas 25: 132-144. https://doi.org/10.1111/j.1601-5223.1939.tb02690.x

Oksala T (1939b) Über die somatische Polyploidie bei Insekten. Annales Entomologici Fennici 5(3): 208-218. 
Oksala T (1943) Zytologische Studien an Odonaten I. Chromosomenverhältnisse bei der Gattung Aeschna mit besonderer Berücksichtigung der postreduktionellen Teilung der Bivalente. Annales Academiae Scientiarum Fennicae (A) IV, Biologica (4): 1-64.

Oksala T (1944) Zytologische Studien an Odonaten. II. Die Enstehung der Meiotischen Präkozität. Annales Academiae Scientiarum Fennicae (A) IV, Biologica (5): 1-33.

Oksala T (1945) Zytologische Studien an Odonaten. III. Die Ovogenese. Annales Academiae Scientiarum Fennicae (A) IV, Biologica (9): 1-132.

Oksala T (1952) Chiasma formation and chiasma interference in the Odonata. Hereditas 38: 449-480. https://doi.org/10.1111/j.1601-5223.1952.tb02937.x

Omura T (1949) On at-random connection of chromosomes in the aeschnid dragonfly, Ictinus rapax. Japanese Journal of Genetics 24: 162-165. https://doi.org/10.1266/jjg.24.162

Omura T (1952) The spermatogenesis of an Indian dragonfly, Ictinus rapax (Rambur) with special reference to the behaviour of the spermatozoa in the cyst. Biological Journal of Okayama University 1(1-2): 103-146.

Omura T (1953) On the abnormal spermatogenesis in an Indian dragonfly, Ictinus rapax (Rambur). Biological Journal of Okayama University 1(3): 163-170.

Omura T (1955) A comparative study of the spermatogenesis in the Japanese dragonflies. I. Family Libellulidae. Biological Journal of Okayama University 2(2-3): 95-135.

Omura T (1957) A comparative study of the spermatogenesis in the Japanese dragonfly II: Family Aeschnidae, Gomphidae and Calopterygidae. Biological Journal of Okayama University 3: 1-86.

Perepelov EA (2003) Karyotype evolution of Odonata (Insecta) of Northern Palearctics. Ph.D. Dissertation, Novosibirsk, Russian Federation: Institute of Systematics and Ecology of Animals of Siberian Branch of Russian Academy of Sciences, 144 pp. [In Russian] https://www. dissercat.com/ content/evolyutsiya-kariotipov-strekoz-insecta-odonata-severnoi-palearktiki

Perepelov E, Bugrov AG (2001a) C-heterochromatin in chromosomes of Ophiogomphus Cecilia cecilia (Four.) (Anisoptera: Gomphidae) with notes on the sex chromosome origin in the species. Caryologia 54(2): 169-172. https://doi.org/10.1080/00087114.2001.10589224

Perepelov E, Bugrov AG (2001b) The constituent geterochromatin in karyotypes of dragonflies. Belyshevia 1(1): 10-13. [In Russian]

Perepelov E, Bugrov AG (2002) Constitutive heterochromatin in chromosomes of some Aeshnidae, with notes on the formation of the neo-XY/neo-XX mode of sex determination in Aeshna (Anisoptera). Odonatologica 31(1): 77-83.

Perepelov EA, Bugrov AG, Warchałowska-Śliwa E (1998) C banded karyotypes of some dragonfly species from Russia. Folia biologica (Kraków) 46: 137-142.

Perepelov EA, Bugrov AG, Warchalowska-Sliwa E (2001) C-banded karyotypes of some dragonfly species from Russia. II. The families Cordulegasteridae, Corduliidae and Gomphidae. Folia biologica (Kraków) 49(3-4): 175-178.

Prasad K, Thomas KI (1992) C-band pattern homogeneity in dragonflies (Odonata). Caryologia 45: 57-68. https://doi.org/10.1080/00087114.1992.10797211

Ray Chaudhuri SP, Dasgupta J (1949) Cytological studies on the Indian dragonflies I. Structure and behaviour of chromosomes in six species of dragonflies (Odonata). Proceedings of the Zoological Society of Bengal 2: 81-93. 
Rehn AC (2003) Phylogenetic analysis of higher-level relationships of Odonata. Systematic Entomology 28: 181-240. https://doi.org/10.1046/j.1365-3113.2003.00210.x

Sandhu R, Malhotra I (1994a) Karyological studies of four aeshnid dragonflies from the states of Jammu and Kashmir and Himachal Pradesh (India). In: Srivastava VK (Ed.) Advances in Oriental Odonatology: Proceedings of IV South Asian Symposium of Odonatology, Allahabad, India October 10-12, 1992, Cherry publications, Allahabad, 111-115.

Sandhu R, Malhotra I (1994b) New chromosome count in male dragonfly, Anatogaster s. basalis. Bionature 14: 69-70.

Sandhu R, Walia GK (1995) A note on the karyotype of Potamarcha congener (Anisoptera: Libellulidae). Chromosome Information Service 58: 24-25.

Sangal SK, Tyagi BK (1982) The spermatocyte chromosomes of Anax immaculifrons Rambur from India (Anisoptera: Aeshnidae). Notulae odonatologicae 1(9): 154-155.

Schorr M, Paulson D (2020) World Odonata List. https://www.pugetsound.edu/academics/academic-resources/slater-museum/biodiversity-resources/dragonflies/world-odonata-list2/

Seshachar BR, Bagga S (1962) Chromosome number and sex-determining mechanism in dragonfly Hemianax ephippiger (Burmeister). Cytologia 27: 443-449. https://doi.org/10.1508/ cytologia.27.443

Seshachar BR, Bagga S (1963) A cytochemical study of oogenesis in the dragonfly Pantala flavescens (Fabricius). Growth 27: 225-246.

Sharma OP, Durani S (1995) A study on the chromosomes of three species of dragonflies (Odonata: Anisoptera). National Academy Science Letters 18(5-6): 97.

Smith EA (1916) Spermatogensis of the dragonfly Sympetrum semicinctum with remarks upon Libellula basalis. Biological Bulletin 31: 269-290. https://doi.org/10.2307/1536236

Souza Bueno AM (1982) Estudos cromossomicos na ordem Odonata. M. Sc. Thesis, Universidad Estatal Paulista, 140 pp.

Srivastava MDL, Das CC (1953) Heteropycnosis in the autosome segments of Ceriagrion coromandelianum (Odonata). Nature 172: 765-766. https://doi.org/10.1038/172765b0

Suzuki KJ, Saitoh K (1990) A revised chromosome study of Japanese Odonates (I). Chromosomes of 14 species belonging to nine families. The Science Reports of the Hirosaki University 37: 38-49.

Suzuki KJ, Saitoh K, Sawano J (1991) Male germ-line chromosomes of Orthetrum poecilops miyajimaensis Yuki et Doi, 1938 (Libellulidae: Odonata). Tombo 34: 29-30.

Toyoshima H, Hirai H (1953) Studies on chromosomes of four dragonflies from Kagawa Prefecture. Kagawa Biology 1: 17-19. [In Japanese]

Thomas KI, Prasad R (1981) The chromosomes of five Indian dragonflies (Odonata). Perspectives in Cytology and Genetics 3: 629-632.

Thomas KI, Prasad R (1986) A study of the germinal chromosomes and C-band patterns in four Indian dragonflies (Odonata). Perspectives in Cytology and Genetics 5: 125-131.

Tyagi BK (1977) A note on the karyotypes of Burmagomphus pytamidalis Laidlow and Onychogomphus saundersi duaricus Faser (Anizoptera; Gomphidae). Odonatologica 6(4): 277-282.

Tyagi BK (1978a) The chromosome numbers and sex-determining mechanisms newly recorded in thirteen Indian dragonflies (Odonata). Chromosome Information Service, Tokyo 25: 5-7. 
Tyagi BK (1978b) Studies on the chromosomes of Odonata of Dun Valley (Dehradun, India). PhD thesis, University of Garhwal, Srinagar.

Tyagi BK (1982) Cytotaxonomy of Indian dragonflies. Indian Review of Life Sciences 2: 149-161. van Brink JM, Kiauta B (1964) Notes on chromosome behaviour in the spermatogenesis of the damselfly Enallagma cyathigerum (Charp.) (Odonata: Coenagrionidae). Genetica 35: 171-174. https://doi.org/10.1007/BF01804885

Walia GK (2007) Cytomorphological studies on Gynacantha milliardi Fraser of the family Aeschnidae (Anisoptera: Odonata). Cytologia 72(1): 57-62. https://doi.org/10.1508/cytologia.72.57

Walia GK, Chahal SS (2014) Distribution of constitutive heterochromatin and nucleolar organizer regions in two species of family Gomphidae (Odonata: Anisoptera). Nucleus 57: 223-227. https://doi.org/10.1007/s13237-014-0122-z

Walia GK, Chahal SS (2018) Cytogenetic characterization of Macromia moorei Selys, 1874 of family Macromiidae (Odonata: Anisoptera) from India by C-banding, silver nitrate staining and sequence specific staining. International Journal of Life Sciences Research 6(2): 64-68.

Walia GK, Chahal SS (2019) Cytogenetic report on Cordulegaster brevistigma and Watanabeopetalia atkinsoni (Odonata: Cordulegastridae, Chlorogomphidae). Odonatologica 48(1-2): 101-113.

Walia GK, Chahal SS (2020) Linear differentiation of chromosomes of Anisogomphus bivittatus Selys, 1854 from India (Odonata: Anisoptera: Gomphidae). International Journal of Entomology 5(2): 120-122.

Walia GK, Chahal SS, Babu R (2016) Cytogenetic report on Gynacanthaeschna sikkima from India (Odonata: Aeshnidae). Odonatologica 45: 87-94.

Walia GK, Chahal SS, Somal DS (2018) Chromosome observations based on C-banding, AgNOR and sequence-specific staining in two Anax species from India (Odonata: Aeshnidae). Odonatologica 47(1-2) 2018: 145-160.

Walia GK, Devi M (2018) Distribution of constitutive heterochromatin in four species of genus Copera of family Platycnemididae (Odonata: Zygoptera) from India. International Journal of Life Sciences 6(2): 457-461.

Walia GK, Devi M (2020a) Cytogenetic characterization of five species of genus Coeliccia of family Platycnemididae (Odonata: Zygoptera) using C-banding, silver nitrate staining and sequence specific staining. Nucleus (2020). https://doi.org/10.1007/s13237-020-00314-3

Walia GK, Devi M (2020b) Cytogenetic data of subfamily Disparoneurinae (Odonata: Zygoptera: Platycnemididae) based on localization of C-heterochromatin, AgNOR's and AT-GC regions. International Journal of Entomology Research 5(2): 70-73.

Walia GK, Katnoria N (2018) Morphological variation in the chromosome complement of Neurobasis chinensis chinensis of family Calopterygidae (Odonata: Zygoptera). International Journal of Life Sciences Research 6(4): 260-266.

Walia GK, Katnoria N, Gill JK (2018) Chromosomes of Libellago lineata lineata (Chlorocyphidae: Odonata). Indian Journal of Entomology 80(3): 737-740. https://doi. org/10.5958/0974-8172.2018.00118.9

Walia GK, Kaur H, Kaur J (2011) Karyotypic variations in the chromosome complement of Pantala flavescens (Fabricius) of the family Libellulidae (Anisoptera: Odonata). Cytologia 76(3): 301-307. https://doi.org/10.1508/cytologia.76.301 
Walia GK, Kaur H, Kaur J (2015) Karyomorphological variations in the chromosome complement of Orthetrum taeniolatum of family Libellulidae (Odonata: Anisoptera). Cytologia 80(1): 95-99. https://doi.org/10.1508/cytologia.80.95

Walia GK, Sandhu R (1999) Karyotypic study of two species of family Aeschnidae (Anisoptera: Odonata). Chromosome Science 3: 45-47.

Walia GK, Sandhu R (2002) Chromosomal data on seven species of genus Orthetrum (Libellulidae: Anisoptera: Odonata). Bionature 22: 7-12.

Walia GK, Sandhu R, Goyal S (2006) Cytogenetical analysis of Nepogomphus modestus from Palampur area of Himachal Pradesh, India (Gomphidae: Anisoptera). Chromosome Science 9(3): 99-100.

Wasscher M (1985) The karyotypes of some dragonflies from Kenya and Sudan. Notulae odonatologicae 2(6): 105-106.

Wolfe LS (1953) A study of the genus Uropetala Selys (order Odonata) from New Zealand. Transactions and Proceedings of the Royal Society of New Zealand 80(3-4): 245-275.

Zhu H, Wu J (1986) Notes on the male germ cell karyotypes of some Odonata from the Shanxi Province, China. Notulae odonatologicae 2: 118-120. 